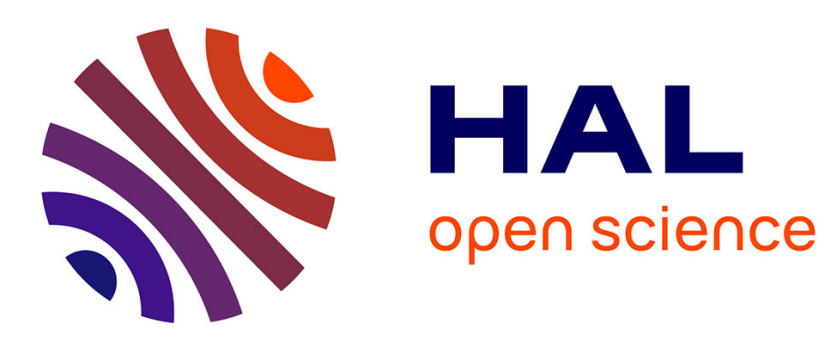

\title{
The atomic and electronic structure of dislocations in Ga based nitride semiconductors
}

\author{
Imad Belabbas, Pierre Ruterana, Jun Chen, Gérard Nouet
}

\section{To cite this version:}

Imad Belabbas, Pierre Ruterana, Jun Chen, Gérard Nouet. The atomic and electronic structure of dislocations in Ga based nitride semiconductors. Philosophical Magazine, 2006, 86 (15), pp.2245-2273. 10.1080/14786430600651996 . hal-00513682

\section{HAL Id: hal-00513682 \\ https://hal.science/hal-00513682}

Submitted on 1 Sep 2010

HAL is a multi-disciplinary open access archive for the deposit and dissemination of scientific research documents, whether they are published or not. The documents may come from teaching and research institutions in France or abroad, or from public or private research centers.
L'archive ouverte pluridisciplinaire HAL, est destinée au dépôt et à la diffusion de documents scientifiques de niveau recherche, publiés ou non, émanant des établissements d'enseignement et de recherche français ou étrangers, des laboratoires publics ou privés. 


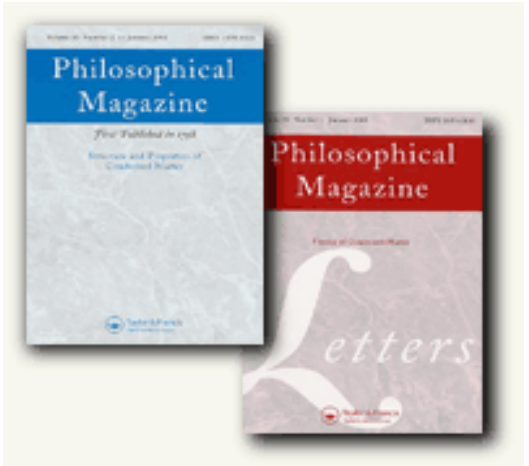

The atomic and electronic structure of dislocations in $\mathbf{G a}$ based nitride semiconductors

\begin{tabular}{|c|c|}
\hline Journal: & Philosophical Magazine \& Philosophical Magazine Letters \\
\hline Manuscript ID: & TPHM-05-Sep-0420.R2 \\
\hline Journal Selection: & Philosophical Magazine \\
\hline $\begin{array}{r}\text { Date Submitted by the } \\
\text { Author: }\end{array}$ & 08-Dec-2005 \\
\hline Complete List of Authors: & $\begin{array}{l}\text { BELABBAS, Imad; ENSICAEN, SIFCOM } \\
\text { Ruterana, Pierre; ENSICAEN, SIFCOM } \\
\text { CHEN, Jun; IUT, LRPMN } \\
\text { NOUET, Gérard; ENSICAEN, SIFCOM }\end{array}$ \\
\hline Keywords: & GaN, atomic structure, dislocations, electronic structure \\
\hline Keywords (user supplied): & \\
\hline
\end{tabular}

\section{ScholaronE \\ Manuscript Central}




\title{
The atomic and electronic structure of dislocations in Ga based nitride semiconductors
}

\author{
I. Belabbas ${ }^{1,3}$, P.Ruterana ${ }^{1}$, J. Chen $^{2}$, G. Nouet $^{1}$ \\ ${ }^{1}$ Laboratoire Structure des Interfaces et Fonctionnalité des Couches Minces, UMR CNRS \\ 6176, Ecole Nationale Supérieure d'Ingénieurs de Caen, 6 Bld du Maréchal Juin, 14050 \\ Caen cedex, France. \\ ${ }^{2}$ Laboratoire de Recherche sur les Propriétés des Matériaux Nouveaux, IUT d'Alençon, \\ 61250 Damigny, France \\ ${ }^{3}$ Groupe de Physique du Solide, Laboratoire de Physique Théorique, Université A.Mira, \\ Béjaia, 06000, Algérie.
}

\begin{abstract}
The atomic and electronic properties of dislocations in III-N semiconductor layers, especially $\mathrm{GaN}$ are presented. The atomic structure of the $\vec{a}$ edge threading dislocation is now well established with three different cores ( 8 or full core, 5/7 or open core and 4 atom ring). The use of atomistic simulations has confirmed these atomic structures and has given a good understanding of the electronic structure of the screw dislocation. Partial dislocations which are mostly confined in the area close to the substrate are now also being investigated. It is becoming clear that the electrical activity of all these defects is dependent on the layer quality, which is governed by the growth conditions.
\end{abstract}




\section{Introduction}

During the last decade, III-V nitride semiconductors have taken an important part in optoelectronic devices since the first commercial light emitting diode (LED) in 1993 fabricated from metal organic vapour phase epitaxy (MOVPE) GaN/InGaN heterostructures containing a very large number of threading dislocations $\left(10^{10} \mathrm{~cm}^{-2}\right)[1]$. These LEDs were reliable although the dislocation density was higher compared to LEDs based on GaAs which emitted in the red region with defect densities below $10^{2}-10^{3} \mathrm{~cm}^{-2}$. The nature and properties of the dislocations in $\mathrm{GaN}$ needed to be understood, and the first reports tended to show that these dislocations should have at least a particular atomic structure which allows them to be non-active in the electronic processes [2]. By conventional transmission electron microscopy (TEM), it was shown that the only dislocations which formed in wurtzite GaN corresponded to the dislocations of the hexagonal lattice with the Burgers vectors $\vec{b}=\vec{a}, \vec{a}+\vec{c}$ and $\vec{c}$ [34]. The non activity of threading dislocations was assumed in the first theoretical report on the atomic structure of the $\vec{a}$ edge threading dislocation; it was proposed that the edge threading dislocation should have either a core based on an 8 atom ring with the dangling bonds reconstructed along the [0001] line or an empty core [5]. At the same time, a theoretical investigation of the motion of the prismatic threading edge dislocations and of the basal screw dislocations in GaN concluded that the mobility of the dislocations in GaN was very small at room temperature, a factor of approximately $10^{-10}$ to $10^{-16}$ with respect to GaAs, which could help to explain the remarkable reliability of GaN based LED [6].

A confirmation of the atomic structure came from the first report of Z-contrast results using high resolution scanning transmission microscopy [7], it was suggested that the $\vec{a}$ edge threading dislocation in $\mathrm{GaN}$ should have the reconstructed 8 atom core structure. In the meantime, extensive experimental work had been done on other extended defects which 
seemed to be typical for the $\mathrm{GaN}$; these were prismatic stacking faults [8] and inversion domains [9-10].

At the same time, the electrical properties investigations were also analysed showing that $\mathrm{GaN}$ nitride layers had quite low carrier mobility due to the presence of the large densities of dislocations [11]. In fact, it was becoming clear that in order to fabricate devices such as lasers in which there is propagation of carriers in the basal plane, the density of threading dislocations needed to be decreased. As a consequence, the first 10000 hours life time laser diode was fabricated in a GaN layer grown using epitaxial lateral overgrowth and having an average threading dislocation density $<10^{8} \mathrm{~cm}^{-2}$ [12]. So, it was necessary to carry out extensive work on the dislocations in order to explain their true behaviour in GaN layers. Simultaneously to the Z-contrast report, our high resolution transmission electron microscopy (HRTEM) investigation showed that the edge threading dislocation had more than the 8 atom core configuration [13]. Mainly, in agreement with the theoretical work of Wright [14] as the open core configuration could not be distinguished with the proposed 5/7 atom structure in HRTEM observations.

During the last few years, a large number of investigations have been carried out on the structure and properties of all types of dislocations in $\mathrm{GaN}$ as well as the possible effect of deviation from stoichiometric composition [15-16] and impurities [17]. Latterly, this effort has also been focused on the partial dislocations and their connections to other extended defects [18-19].

In this paper, we discuss the numerous results that have been reported along with our contribution on experimental HRTEM and theoretical investigation of these defects. Typically, our work has allowed to determine the multiple configuration of the threading edge dislocation and to clarify the crystallographic relationships between them [20-21]. The related study of electronic properties is a topic of high interest and different methods have been used 
to calculate the energy and electronic structure of defects in semiconductors. Empirical potentials still provide guidance in the most complex situations where large sets of atoms need to be used, but they cannot give any information on the electronic properties due to their analytical characteristics chosen to fit with elastic or phonon properties. The tight-binding theory has addressed an important field of computational materials science; it is a reasonable approach for the calculation of atomic and electronic structures in covalently bonded materials. In this vein, the density functional theory (DFT)-based tight-binding (DFTB) method operating in a self-consistent charge mode has offered a robust and transferable method to model a semiconductor crystal on the atomic scale [22]. Indeed, in the case of dislocations in $\mathrm{GaN}$, insight has been gained in the most recent approaches which are multiscale, going from the empirical potentials to deal with large sets of atoms, to ab-initio modelling of the core of area [23]. In GaN, many configurations of the dislocations have now been investigated using these methods, and their atomic and electronic structures are reported on and discussed here. In a first part, we describe the crystallography of the structure and mainly of the dislocations. The following part deals with the experimental analysis of prismatic and basal dislocations, and their electrical behaviour. In the end, we discuss the atomistic simulation of the dislocation cores, and the calculations of their electronic structures.

\section{Crystallographic data}

\subsection{Structure}

Gallium nitride, GaN, crystallises in the cubic system with the zincblende form (sphalerite) or in the hexagonal system (wurtzite) [24]. For both forms, atoms have a tetrahedral coordination and the ideal angle between bonds is $109^{\circ} 5$. The sphalerite structure 
(a)

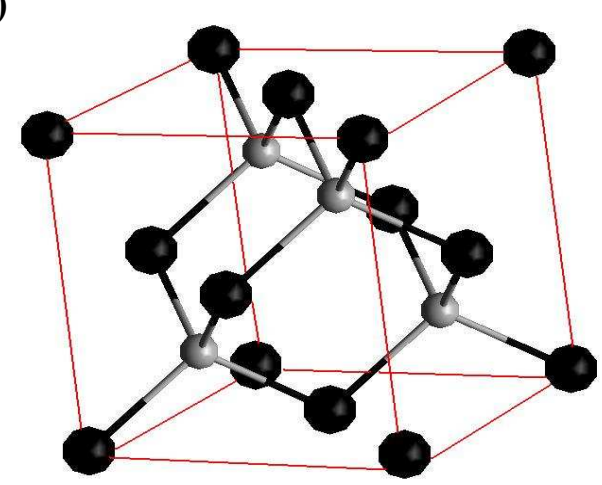

(b)

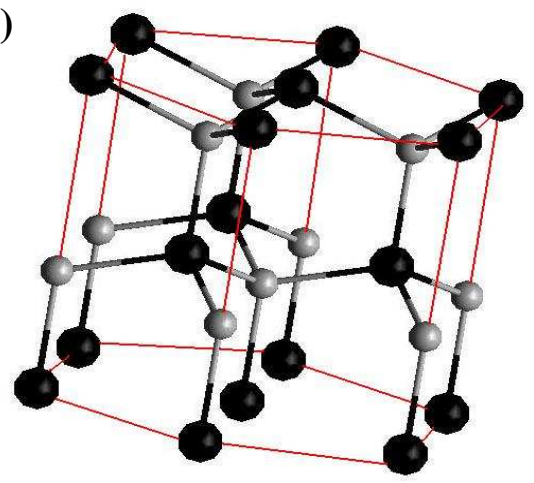

Fig.1: Structures of zinc blende and wurtzite crystals. a) zinc blende unit cell. b) wurtzite unit cell ( Ga: large black spheres, N: small grey spheres)

The zinc blende structure has only one type of first neighbour distance, which means that the four bond lengths in a tetrahedron are equal. This is also valid for a wurtzite structure with the ideal values $c / a=1.633$ and $u=3 / 8$. Otherwise, the bond parallel to the $c$ axis is 
different, within less than $1 \%$ for the III-V nitrides. For the second nearest neighbours, the zinc blende structure has only one type of bond lengths (12 bonds). The wurtzite structure presents three types of second neighbours, and for the ideal values, the difference between the shortest and the longest bonds is $13 \%$ [25].

\subsection{Dislocations}

In the frame of the linear elasticity theory of isotropic materials, the displacement field of the dislocation can be calculated as well as the induced stresses, except in the vicinity of the dislocation line or dislocation core where both stress and strain become infinite and this analysis breaks down. The strain energy of an infinite straight dislocation in a perfect crystal is calculated per unit length when the dislocation is contained in a cylinder of radius R. For a screw or an edge dislocation, this stored elastic energy is proportional to the square of the Burgers vector, $b^{2}$. The ratio of their energies is equal to the factor $(1-v), v$ is the Poisson's ratio, and the energy of an edge dislocation is larger than that of a screw dislocation. The total energy of the dislocation also includes the energy stored in the dislocation core. In the linear elastic theory, the displacements vary slowly; this assumption breaks down in the core region and atomistic calculations become necessary to calculate the corresponding energy.

Since the elastic energy of a dislocation is proportional to the square of the Burgers vector, this value of $b^{2}$ may be used to define the stability of a dislocation by applying the Frank criterion to the splitting of a dislocation with Burgers vector $\vec{b}_{1}$ into dislocations $\vec{b}_{2}$ and $\vec{b}_{3}$. The splitting takes place only if: $\left(\mathrm{b}_{1}\right)^{2}>\left(\mathrm{b}_{2}\right)^{2}+\left(\mathrm{b}_{3}\right)^{2}$. Applying this criterion, the possible Burgers vectors are only the primitive translations of the lattice. As a consequence, the Burgers vectors of the perfect dislocations in the zinc blende and wurtzite structures are identical to those of diamond or hexagonal close-packed material, respectively [26] (Table 1). 


\begin{tabular}{|c|c|c|c|}
\hline \multicolumn{2}{|c|}{ Zinc blende } & \multicolumn{2}{c|}{ Wurtzite } \\
\hline Burgers vector $(\vec{b})$ & $\left(\mathrm{b}^{2}\right)$ & Burgers vector $(\vec{b})$ & $\left(\mathrm{b}^{2}\right)$ \\
\hline $1 / 2<110>$ & $\mathrm{a}^{2} / 2$ & $1 / 3<1 \overline{120}>$ & $\mathrm{a}^{2}$ \\
\hline$<100>$ & $\mathrm{a}^{2}$ & $<0001>$ & $\mathrm{c}^{2}$ \\
\hline & & $1 / 3<1 \overline{12} 3>$ & $\mathrm{a}^{2}+\mathrm{c}^{2}$ \\
\hline
\end{tabular}

Table 1: Perfect dislocations in zinc blende and wurtzite structures: Burgers vector $(\vec{b})$ and energy $\left(b^{2}\right)$.

By considering the link between the crystallographic structure of crystals and the structure of dislocations, dislocations with Burgers vectors not belonging to lattice translations must be present. Such dislocations are defined as partial dislocations and the area connecting two partial dislocations is faulted (Table 2). As a first approximation, the application of the Frank criterion shows that the dissociation of a perfect dislocation into partials may take place.

\begin{tabular}{|c|c|c|c|c|c|}
\hline \multicolumn{3}{|c}{ Zincblende } & \multicolumn{3}{c|}{ Wurtzite } \\
\hline Type & Burgers vector $(\vec{b})$ & $\left(\mathrm{b}^{2}\right)$ & Type & Burgers vector $(\vec{b})$ & $\left(\mathrm{b}^{2}\right)$ \\
\hline Shockley & $1 / 6<112>$ & $\mathrm{a}^{2} / 6$ & Shockley & $1 / 3<10 \overline{1} 0>$ & $\mathrm{a}^{2} / 3$ \\
\hline Frank & $1 / 3<111>$ & $\mathrm{a}^{2} / 3$ & Frank & $1 / 2<0001>$ & $\mathrm{c}^{2} / 4$ \\
\hline \multirow{3}{*}{} & $1 / 6<110>$ & $\mathrm{a}^{2} / 18$ & Frank-Shockley & $1 / 6<20 \overline{2} 3>$ & $\mathrm{a}^{2} / 3+\mathrm{c}^{2} / 4$ \\
\cline { 2 - 3 } & $1 / 3<001>$ & $\mathrm{a}^{2} / 9$ & & \\
\cline { 2 - 3 } & $1 / 3<110>$ & $2 \mathrm{a}^{2} / 9$ & & & \\
\cline { 2 - 3 } & $1 / 6<301>$ & $5 \mathrm{a}^{2} / 18$ & & & \\
\hline
\end{tabular}

Table 2: Partial dislocations in zinc blende and wurtzite structures: Burgers vector $(\vec{b})$ and energy $\left(b^{2}\right)$.

For the nitrides, the perfect dislocations that have been characterised are the prismatic dislocation or edge dislocation $\vec{b}=1 / 3<11 \overline{2} 0>, l=<0001>$ with three different atomic cores and the screw dislocation $\vec{b}=<0001>, l=<0001>$ with two atomic cores (Fig.2).
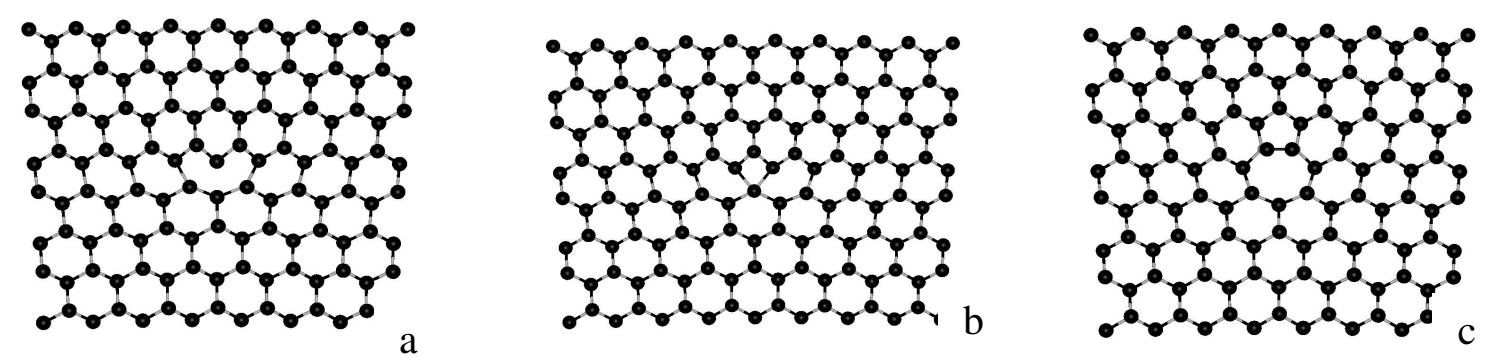

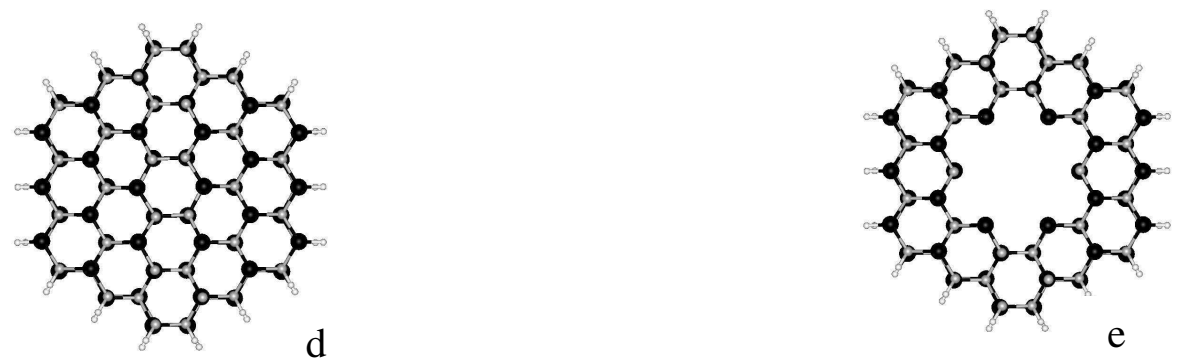

Fig.2: Prismatic dislocation or edge dislocation $\vec{b}=1 / 3<11 \overline{2} 0>, l=<0001>$ a: Full core or 8 atom ring, b: 4 atom ring, c: Open core or 5/7 atom ring and screw dislocation $\vec{b}=<0001>, l=<0001>$ d: Full core, e: Open core

In the basal plane, they may be $60^{\circ}$ (the angle between the line direction and the Burgers vector is $\left.60^{\circ}\right), \quad \vec{b}=[11 \overline{2} 0], l=[2 \overline{1} \overline{1} 0]$ or screw dislocations, $\vec{b}=[11 \overline{2} 0], l=$ $[11 \overline{2} 0]$

\section{Experimental observations}

\subsection{Prismatic dislocations}

The heteroepitaxially grown GaN layers on (0001) sapphire substrates whatever the growth process, e.g. molecular beam epitaxy (MBE) or metal organic chemical vapour deposition (MOCVD), are characterised by high densities of threading dislocations running across the layer from the layer/substrate interface to the top surface with their line mainly parallel to the $<0001>$ growth direction [27]. This density is in the order of $10^{9} \mathrm{~cm}^{-2}$ in conventional growth and can go up to $10^{11} \mathrm{~cm}^{-2}$ whatever the growth method; pure edge dislocation $(\vec{b}=1 / 3<1 \overline{120}>, l=<0001>)$ and mixed dislocation $(\vec{b}=1 / 3<11 \overline{2} 3>, l=<0001>)$ concentrations may be comparable and the fraction of pure screw dislocations $(\vec{b}=<0001>, l$ $=<0001>$ ) is usually low: 0.1 to $1 \%$ (Fig. 3) [28]. 

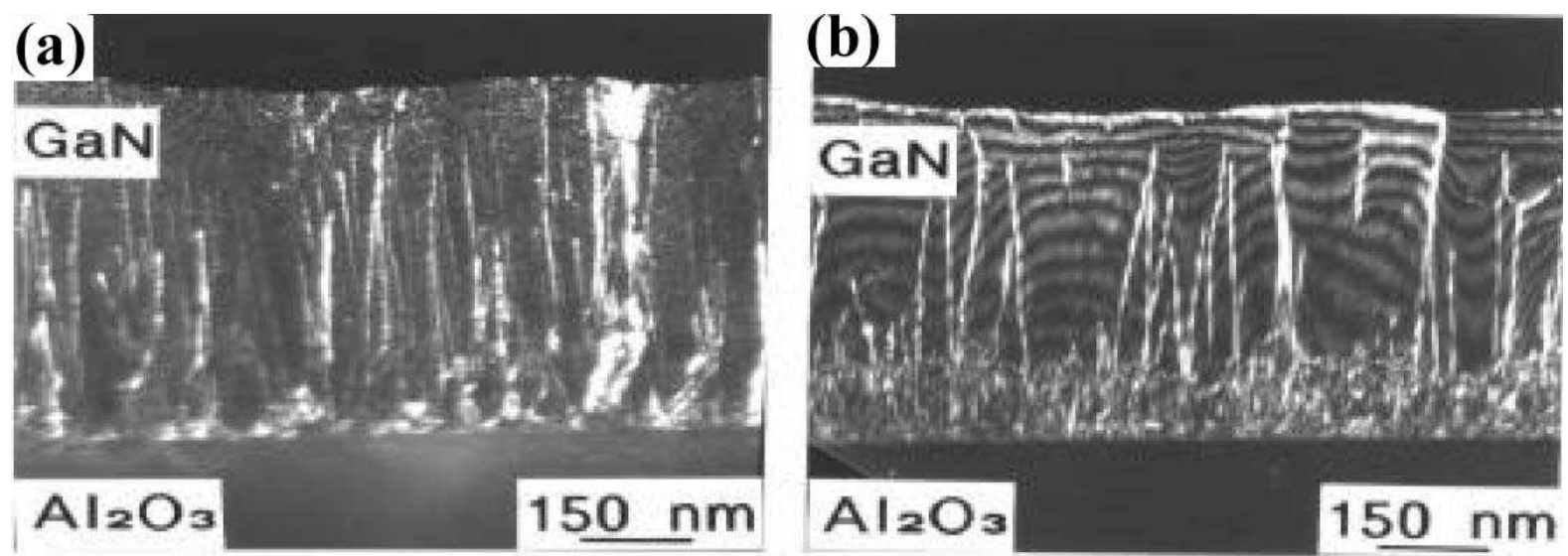

Fig. 3: Typical threading dislocations in MBE GaN layers grown on sapphire:

$$
\text { dark field a) } g=1 \overline{2} 0 \text { b) } g=0002 \text { [29] }
$$

\subsubsection{Edge or mixed threading dislocations}

The first observations of these threading dislocations by HRTEM showed that in good quality $\mathrm{GaN}$ layers grown by $\mathrm{MBE}$ at $800^{\circ} \mathrm{C}$ on (0001) sapphire, two atomic configurations coexisted: the 8 atom core and a 5/7 atom core (Fig.4) [13].

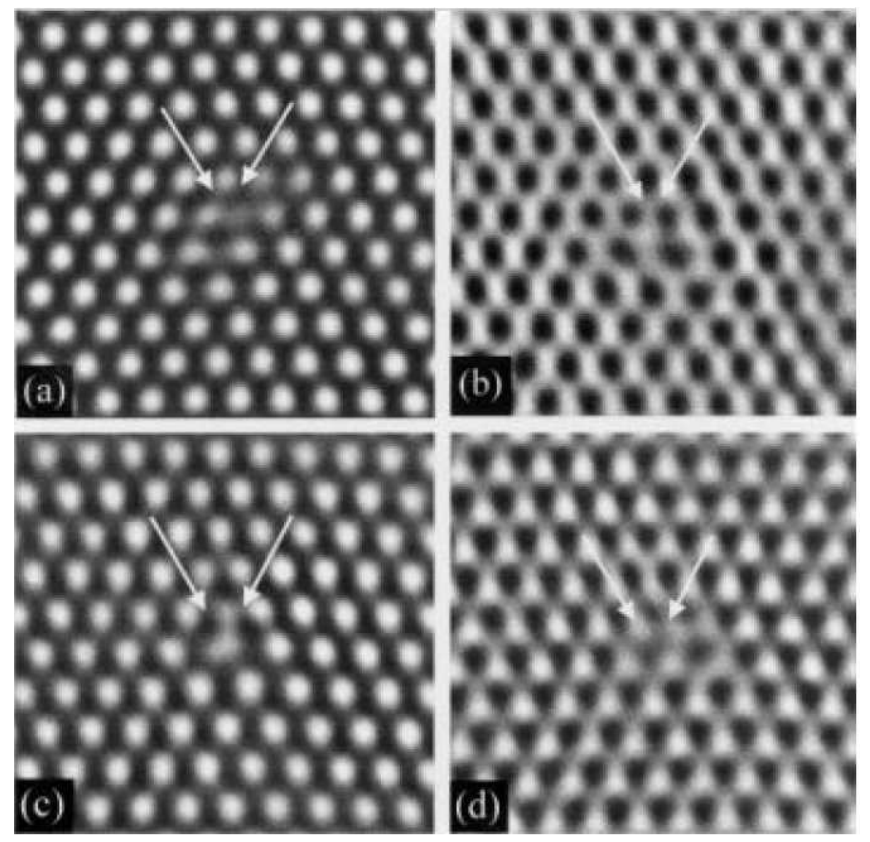

Fig. 4: HREM images along <0001> of the $1 / 3<1 \overline{12} 0>$ edge dislocation: 8 atom ring ( $\mathrm{a}$ and $\mathrm{b}$ ), $5 / 7$ atom ring ( $c$ and $d$ ), (defocus values, a and c:-24nm, b and d:-54nm) [18]

Later, in the investigation of low and high angle grain boundaries [18], it was shown that a configuration with a 4 atom core is also present in the GaN layers (Fig.5). The identification of the atom cores is based on the analysis of experimental and simulated contrasts of the 
images [30]. This was shown to be in agreement with the atomistic calculations of stoichiometric configurations [20].

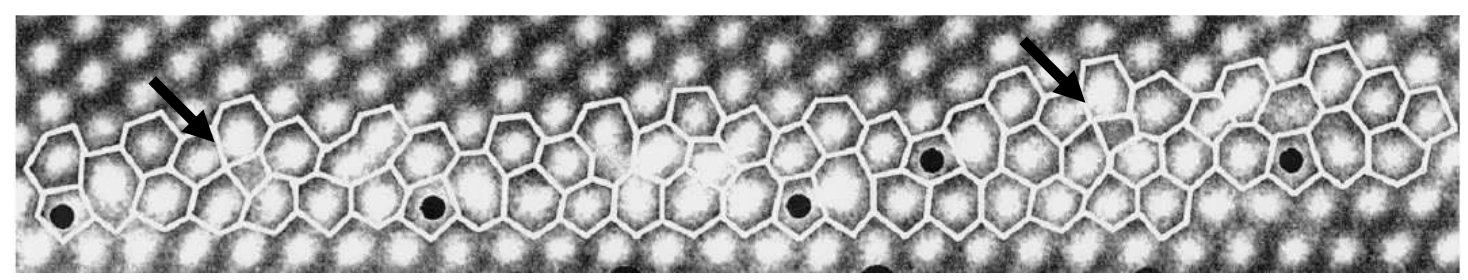

Fig.5: Occurrence of 4 atom ring configurations (arrows) for the $1 / 3<11 \overline{2} 0>$ edge dislocation observed in a non symmetric $\Sigma=7$ grain boundary (Black dots delimit the unit periods of $\Sigma=7$, and the white lines the different atom rings: 6 for the perfect crystal, 5/7, 8 and 4 for the cores of the $1 / 3<1 \overline{2} 0>$ edge dislocation) [18].

Simultaneously, a report was issued using High Angle Annular Dark Field (HAADF) (Zcontrast) imaging showing that the edge dislocation atomic structure was made of 8 atom core [31]. It was argued that such a core should be the only one occurring in GaN in agreement with the theoretical predictions that tried to explain the good electrical and optical properties of GaN layers. For such low electrical activity, the three-fold coordinated atoms in the core had to form dimers along the $<0001>$ line direction.

In epitaxial $\mathrm{GaN}$ layers grown at $800^{\circ} \mathrm{C}$ on a $\mathrm{GaN}$ or $\mathrm{AlN}$ buffer layer deposited at $550^{\circ} \mathrm{C}$, low-angle and high angle grain boundaries were found and analysed (Fig. 6) [32]. By electron diffraction, grain boundaries were identified with rotation angles in the range $1.5-22^{\circ}$. The dislocations were still $1 / 3<1 \overline{12} 0>$ edge type as deduced from the use of the invisibility criterion $\vec{g} \cdot \vec{b}=0$ ( $\vec{g}:$ diffraction vector, $\vec{b}$ : Burgers vector).

Three high angle grain boundaries crossing all the GaN layers were perfectly analysed from HRTEM images by combining topological theory [33] and image simulation [30]. They were described by rotations around the $<0001>$ direction and the values of the rotation angle, as determined from the diffraction patterns, were $13.2 \pm 0.2^{\circ}, 19 \pm 0.2^{\circ}$, and $22.0 \pm 0.2^{\circ}$ [18]. It is well known that the atomic structure of low angle grain boundaries is periodic and based on a 
regular array of dislocations. This concept was then extended to specific high angle grain boundaries, establishing that the atomic structure of coincidence grain boundaries is also periodic [34].

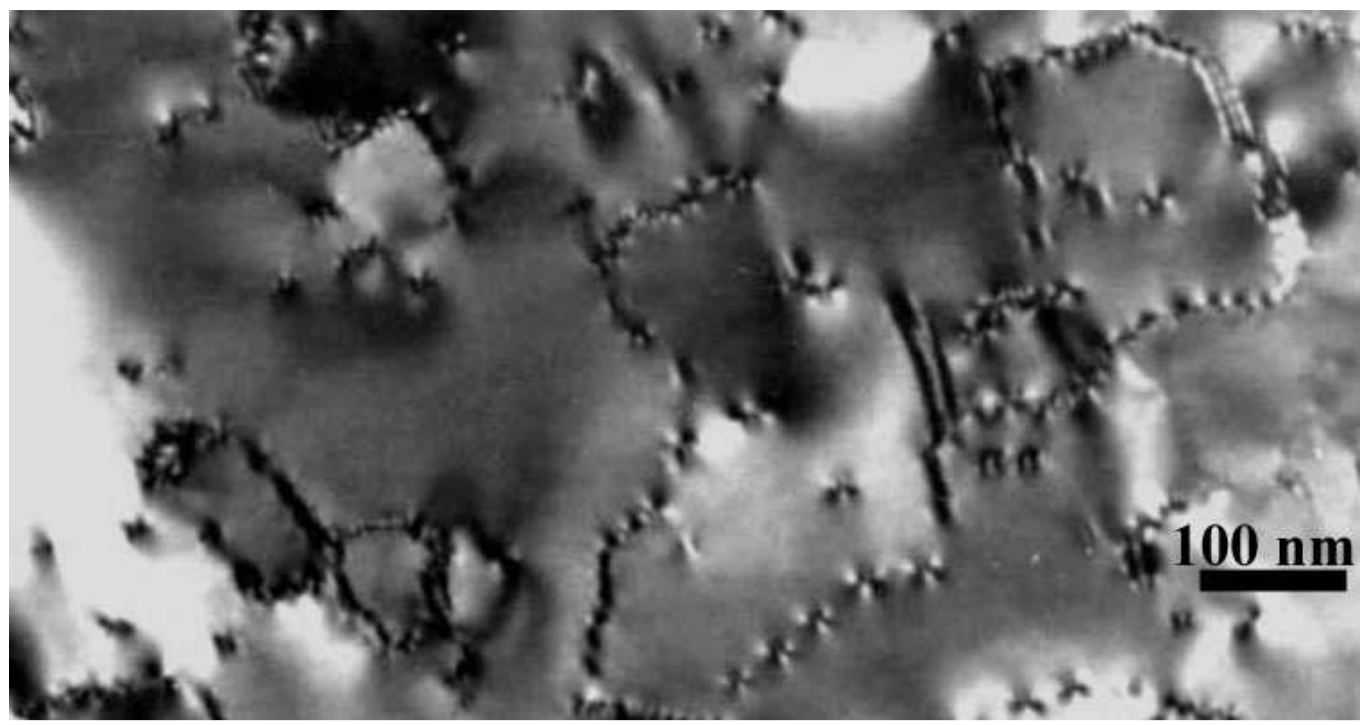

Fig.6: Plan view along [0001] direction showing arrays of dislocations forming low and high angle grain boundaries [32].

These coincidence grain boundaries are defined for specific values of the axis and of the angle of rotation giving rise to a 3D superlattice, the Coincidence Site Lattice (CSL), common to the two adjacent crystals. In the hexagonal system, for rotations around $<0001>$, angles of $13.17^{\circ}, 17.90^{\circ}$ and $21.79^{\circ}$ lead to CSLs which are characterized by their periodic unit cells [35]. The common characteristic of the atomic structures of these coincidence grain boundaries is the regular array of the cores of the edge dislocation $1 / 3<1 \overline{2} 0>$. In Fig.5, between two black dots delimiting the CSL unit cell, 6 atom rings corresponding to the perfect crystal are combined with other atom rings. According to the contrast observed in the HRTEM image and by comparison with the simulated images, the cores were identified as 4 , $5 / 7$ or 8 atom rings. These investigations have allowed us to show that the above dislocation cores are the only structural units, which in addition to the 6 atom unit of the hexagonal 
lattice, can be present in all the possible grains boundaries that form by a rotation around the [0001] direction in $\mathrm{GaN}[18,35]$.

Other extended defects have been shown to exhibit a dislocation character with larger Burgers vectors than unitary lattice parameters. In figure 7 , the circuit around the defect exhibits a closure failure, corresponding to a basal component equal to $2 \vec{a}$ [36]. These defects were identified as nanopipes in comparison to the micropipes observed in $\mathrm{SiC}$ [37], but with rather small radii in contrast to the previsions of the Frank formula [38].

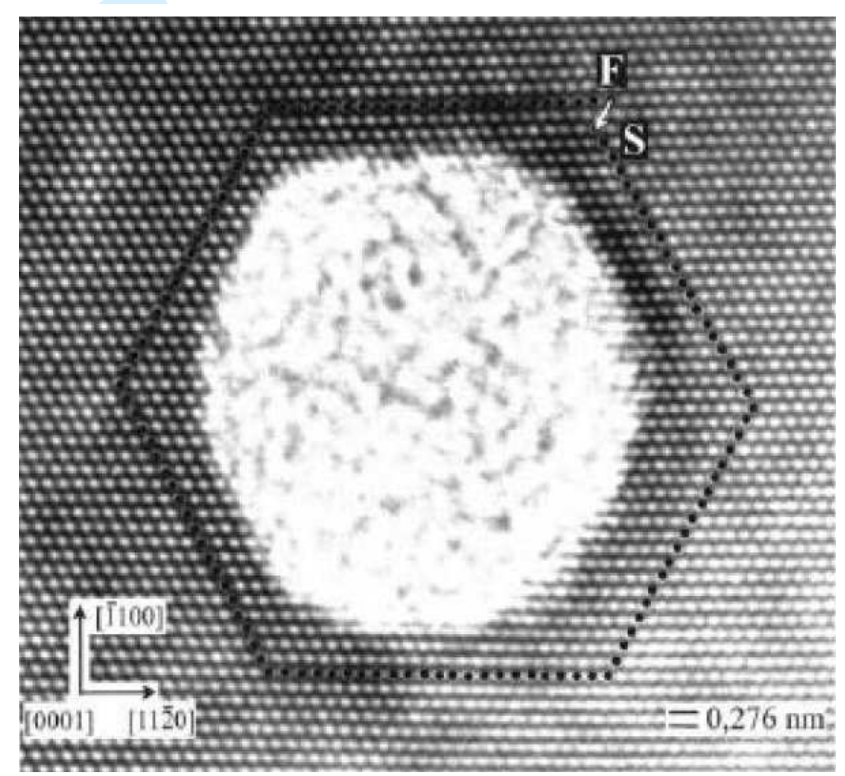

Fig.7: A nanopipe with a Burgers edge component of $2 \vec{a}$ [36]

In conclusion, the atomic structures of the cores of the $1 / 3<1 \overline{12} 0>$ prismatic edge dislocations in GaN analysed by HRTEM combined with simulated images present a multiple atomic configuration: three atomic structures were identified ( 8 atom ring or full core, 5/7 atom ring or open core and 4 atom ring). The 8 atom ring or full core was the only one identified by HAADF. 


\subsubsection{Screw prismatic dislocations}

The first investigations of the surface of GaN layers, grown by MOCVD at $1040^{\circ} \mathrm{C}$ on an AlN buffer layer deposited at $450-500^{\circ} \mathrm{C}$, using scanning force microscopy have shown the presence of tunnel-like defects aligned along the $<0001>$ growth direction. The radii of theses tunnels are in the $3.5-50 \mathrm{~nm}$ range and they propagate along the growth direction. These defects are called nanopipes. These experimental investigations have also shown that the screw dislocation associated with a nanopipe emerges on the top surface as a wide crater with 60-100 nm radii. By combining these observations with HRTEM analysis, it was concluded that the nanopipes are the open cores of screw dislocations $(\vec{b}=0001)$ [39]. This result was later confirmed by showing that such defects may alternate from an open (nanopipe) to closed core along their way to the surface of the epitaxial layer (classical character of a dislocation) [40]. The atomic structure of full core screw dislocations was reported in a high resolution Zcontrast analysis [31]. The growth mode can affect the dislocation core and as was shown in hydride vapour phase epitaxy (HVPE) grown materials, screw dislocations are decorated by voids or pinholes [41]. The fluctuations in the intensity of the atomic columns in HRTEM of the HVPE specimens have been attributed to local excess of gallium atoms in the core [42].

\subsection{Basal dislocations}

GaN layers deposited on top of the (0001) sapphire are characterised by the well known epitaxial relationship: $(0001)_{\mathrm{sap}} / /(0001)_{\mathrm{GaN}}$ and $[1 \overline{12} 0]_{\mathrm{sap}} / /[10 \overline{1} 0]_{\mathrm{GaN}}[43]$. The network of anions is continued across the interface. The misfit is large (-16.07\%) and it is accommodated by misfit dislocations in the basal plane. The possible Burgers vectors of the perfect dislocations are $1 / 3<1 \overline{2} 0>$, and the line directions are $<1 \overline{2} 0>$ leading to pure screw or $60^{\circ}$ dislocations according to the angle between the Burgers vector and the line direction. 
After Bragg filtering of HRTEM images, it was shown that this lattice misfit is relaxed in the first monolayer of $\mathrm{GaN}$ with a network of regularly spaced $60^{\circ}$ dislocations. A residual stress of $-2.1 \%$ was evaluated near the interface and related to the high density of threading dislocations present in the epitaxial layer [44].

Since threading and misfit dislocations are always present, investigations were carried out at an interface between AlN and sapphire in order to analyse the connection of these two dislocation networks [45]. Molecular Beam Epitaxy (MBE) was used for the deposition of the AlN buffer on $\mathrm{Al}_{2} \mathrm{O}_{3}$, at $800^{\circ} \mathrm{C}$, until a thickness of $40 \mathrm{~nm}$ was reached. In HRTEM plan view images, a set of three-fold moiré patterns due to the overlap of the $\{1 \overline{010}\}_{\text {AIN }}$ and $\{1 \overline{120}\}_{\text {sap }}$ planes is formed at the layer-substrate interface with a mean spacing of $2.16 \mathrm{~nm}$ instead of $2.029 \mathrm{~nm}$ for the theoretical value. This discrepancy means that $7 \%$ of the compressive stress is not relaxed by the misfit dislocations. From the moiré pattern, the occurrence of threading dislocations is readily deduced from the terminating moiré fringes (Fig.8).

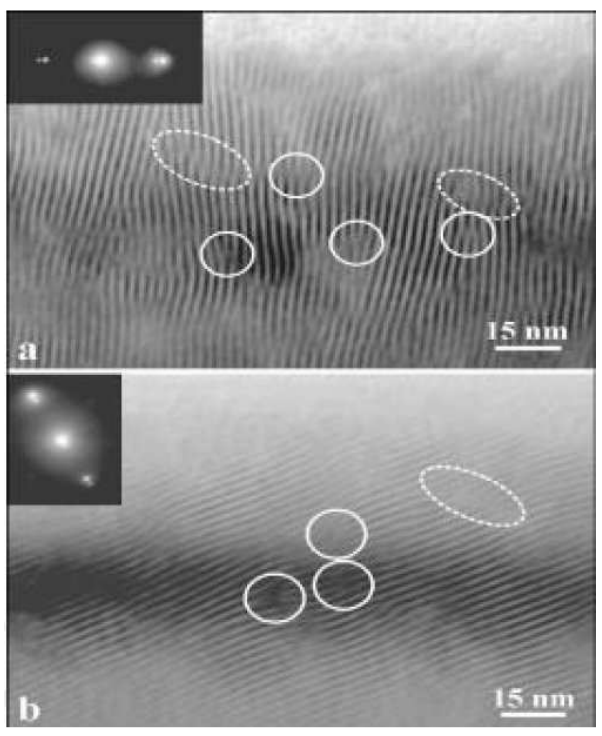

Fig.8: Plan-view HRTEM image showing a translation moiré pattern (a) one set of reflections, (b) a second equivalent set [45]

The dislocation density measurements from various areas of the moiré pattern showed an average density in the order of $1.5 \times 10^{11} \mathrm{~cm}^{-2}$ with a lower rate near the top surface of $8 \times 10^{10}$ $\mathrm{cm}^{-2}$. In figure 9 , the white arrows indicate the terminating fringes due to the threading 

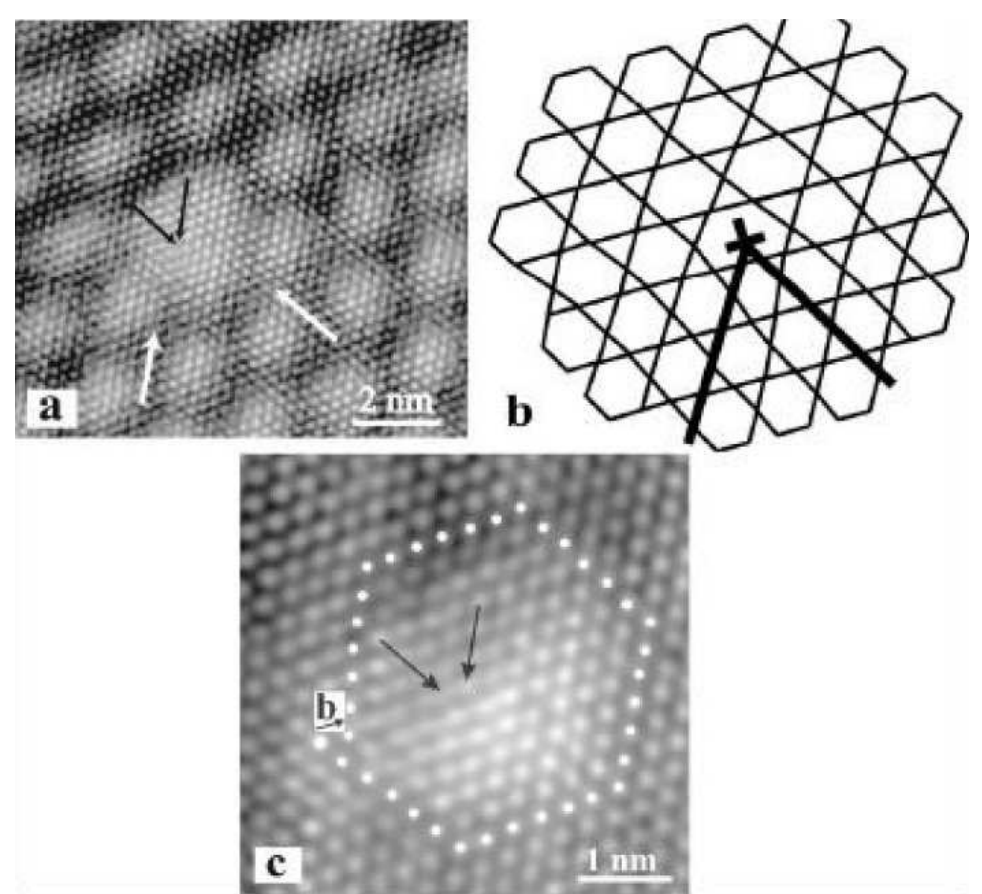

dislocation marked by the black fringes and corresponding to two extra prismatic half planes. The circuit around the defect gives as Burgers vector $\vec{a}$ suggesting an edge or mixed type dislocation.

Fig.9: (a) Threading dislocations imaged by the terminating moiré fringes

(b) Schematic illustration of the misfit network

(c) Fourier filtered image showing only the AlN structure [45]

The corresponding missing half-planes are associated with two misfit dislocations of the interfacial network as shown in Fig.9b. The Burgers vector of each threading dislocation is equal to the algebraic sum of the Burgers vectors of the two associated misfit dislocations. Similar observations with moiré analysis were carried out on HVPE grown layers on SiC substrates [46]. Therefore, threading dislocations existing in $\mathrm{GaN}$ films can be related to the misfit dislocations at the interface with the substrate. This view is complemented by other investigations which show that many threading dislocations can originate inside the buffer layer due to the recombination of stacking faults and subsequent climb of the dislocations in the prismatic planes [47]. 


\subsection{Partial dislocations}

In MOCVD layers grown on (0001) sapphire, evidence of dissociation in the basal plane was shown using the weak beam technique and the following mechanism was proposed: $\vec{c}+\vec{a}=\left(\vec{c} / 2+\vec{b}_{1}\right)+\left(\vec{c} / 2+\vec{b}_{2}\right)$, with $\vec{b}_{1}$ and $\vec{b}_{2}$ being Shockley partials, corresponding to the formation of an intrinsic stacking fault $\mathrm{I}_{1}$ (Ponce et al., 1996). However, after introduction of dislocations by indentation, dissociation of the perfect screw dislocation or $60^{\circ}$ dislocation into partial dislocations was not observed in the limit of the weak beam resolution, $2 \mathrm{~nm}$, however stacking faults were present in the nodes formed by the screw dislocations [48]. In hexagonal structures, three basal faults are possible: two intrinsic $\mathrm{I}_{1}$ and $\mathrm{I}_{2}$ and one extrinsic $\mathrm{E}$. $\mathrm{I}_{1}$ is formed by removing the B plane above the A plane and shearing the remaining part by $1 / 3<10 \overline{1} 0>, I_{2}$ is formed by considering only the shear, and E by inserting one C plane between two adjacent basal planes. The energy of the basal stacking faults was calculated using DFT: $\mathrm{I}_{1}=18, \mathrm{I}_{2}=43$ and $\mathrm{E}=69 \mathrm{~mJ} / \mathrm{m}^{2}[49,50]$. They are of the low-energy type. In HRTEM, the density of stacking faults near the interface with the substrate is very high, most of them are of the $I_{1}$ and $I_{2}$ types even if segments of the $E$ fault were also observed and partial dislocations were identified.

The $\mathrm{I}_{1}$ fault was interpreted as the climb-dissociation process:

of the $1 / 3<1 \overline{120}>$ following the reaction:

$$
1 / 3<1 \overline{12} 0>1 / 6<20 \overline{2} 3>+1 / 6<02 \overline{2} \overline{3}>
$$

or of the $<0001>$ perfect dislocation:

$$
<0001>\quad \rightarrow \quad 1 / 6<20 \overline{2} 3>+1 / 6<2 \overline{2} 03>\text {. }
$$

The $\mathrm{I}_{2}$ fault results from the shear of the structure leading to a partial dislocation loop, the two partial dislocations bounding the basal fault have opposite Burgers vectors $1 / 3<10 \overline{1} 0>$ [51]. Partial dislocations, $1 / 3<10 \overline{10}>$, can also be formed at the intersection of a basal stacking 


\subsection{Electrical properties of the threading dislocations in GaN}

Seeing the high brightness of the first GaN based devices which contained a very large number of threading dislocations, it has first been assumed that the electrical activity should be taken as substantially low. In the last ten years, a number of reports have shown that this is not the case after the use of two techniques: the scanning probes in the atomic force microscope that mostly analyse the properties of the individual defects close to the sample surface, and cathodoluminescence (CL) in TEM.

\subsubsection{Scanning probe microscopy investigations}

In their leakage current comparative work on $\mathrm{GaN}$ based devices from layers grown by HVPE and MBE, Hsu et al. showed that the reverse bias leakage was not uniformly distributed in the sample [53]. A small portion of the sample was found to be responsible for the majority of the leakage. The correlation between reverse bias leakage locations and topographic hillocks in samples suggested that reverse bias leakage primarily occurred at screw/mixed dislocations. Further confirmation was given from the similar density between the leakage spots and screw/mixed dislocation density determined by TEM. The fact that samples had distinctly different morphologies but showed similar density of leakage spots led to the conclusion that the origin of the reverse bias leakage was the same in the set of samples. They showed that screw/mixed dislocations contribute significantly more to gate leakage than 
pure edge dislocations. This was in agreement with other reports where screw/mixed dislocations had been found to be more effective recombination centers [54], and to have reduced barrier heights compared to pure edge dislocations [55]. In such devices, the reverse bias leakage can be orders of magnitude different for samples grown under slightly different conditions. For example, the reverse bias current was orders of magnitude higher for Ga-rich samples. Given that dislocations with a screw component are responsible for the reverse bias leakage and their density is the same for the HVPE template and the two MBE/HVPE films, these results led to conclude that the dislocation electrical activity was sensitive to local chemical and/or structural changes, which depend on growth methods and conditions.

\section{4. 2. Cathodoluminescence investigations}

Correspondence between the individual dislocations and the active centres in MOVPE GaN layers by combining TEM and cathodoluminescence has shown that the dislocations are non-radiative recombination centers [56]. Remmele et al. [57] were able to distinguish the $\vec{a}$ threading dislocations and $\vec{a}+\vec{c}$ dislocations in their bright field images, since the $\vec{c}$ component of the $\vec{a}+\vec{c}$ dislocations caused a black and white lobe contrast due to surface relaxation effects [58]. The $\vec{a}$ dislocations showed only a dark contrast. The comparison of the bright field images and the monochromatic CL mapping (band edge luminescence) gave the following results: (i) the major part of the $\vec{a}+\vec{c}$ dislocations is not recombination active. (ii) $\vec{a}$ threading dislocations reduced the band edge luminescence, thus representing centres of non-radiative recombination, (iii) $\vec{a}$ dislocations in the basal plane caused non-radiative recombination.

\section{Atomistic simulation of dislocations in GaN}

The main goal of atomistic modelling of dislocations is to find realistic models of the cores and provide information on the microscopic mechanisms occurring in this area. From such studies one expects to derive, at least, two types of information. The first is to identify 


\subsubsection{The ab initio methods}

In the ab initio methods, the interatomic interactions are described rigorously in the framework of quantum mechanics. Among the ab-initio methods, those based on DFT are largely used in the field of computational materials science $[59,60]$. In these latter methods, the electronic density plays a key role; the quantities of interest in atomistic simulation: energy and forces are derived from it. The electronic density is obtained by solving the selfconsistent Kohn-Sham equations [60]. To perform ab initio DFT calculations in a tractable way, a number of approximations are necessary. The most important are made on the form of the exchange and correlation functional (Local Density Approximation, Generalised Gradient Approximation ...etc.) and on the basis set used to represent the single-electron wave function (plane waves, Gaussian type functions, Slater type functions). Moreover, to make these calculations more tractable, the pseudopotential approximation is usually used [61]. Despite these approximations, the ab initio based DFT calculations remain computationally expensive and their applications are limited to systems of a few hundred atoms (typically 200 atoms). 
Ab-initio-DFT methods using pseudopotentials were implemented in several codes like: VASP (Vienna Ab-initio Simulation Package) [62], FHI-md (Fritz-Haber Institute's Molecular Dynamics code) [63], AIMPRO (Ab Initio Modelling PROgram) [64] and SIESTA (Spanish Initiative for Electronic Simulations with Thousands of Atoms) [65]. While the first two use a representation based on the plane waves, AIMPRO and SIESTA use Gaussian-type and numerical Slater-type atomic orbital, respectively. Except SIESTA, these codes have been used in different investigations of dislocations in GaN: VASP [15, 16, 66], FHI-md [23] and AIMPRO $[2,22,67,68]$.

\subsubsection{The empirical potentials}

Less sophisticated than ab initio and tight-binding methods, the empirical potentials allow dealing with systems of many thousands of atoms, and this of interest for dislocation modelling [69]. In empirical potential methods, one postulates a relatively simple analytical form of the potential energy including a set of adjustable parameters. These are fitted to experimental measurements or ab initio calculations in order to reproduce a set of properties of the material under investigation (lattice parameter, elastic constants, bulk modulus, phonon spectrum,). It is assumed that the approximated form of the empirical potential will reproduce the main feature of the atom-atom interactions and that the number of adjustable parameters is sufficient to ensure its transferability.

Some empirical potential have previously been used to calculate the energies of dislocations and grain boundaries in semiconductors [70-73]. They brought about a good insight on the intrinsic properties of elemental semiconductors. Keating and Baraff potentials are limited by their parameterisations which exclude dangling bonds. However, the two other potentials: Stillinger-Weber and Tersoff, take into account different atomic surroundings. The Stillinger-Weber potential has been used for III-V compound semiconductors [20, 74-76]. Of 


\subsubsection{The tight-binding methods}

Coming right in between the $\mathrm{ab}$ initio and empirical potential methods, tight-binding methods combine a minimal amount of quantum mechanics of electrons with classical potentials. This allows achieving more transferable description of the interatomic interactions than empirical potentials, at a small fraction of the computational cost of ab-initio calculations. Consequently, tight-binding methods are appropriate to model systems with many hundred to a thousand atoms and can be applied to deal with problems beyond the reach of ab-initio methods. In standard tight-binding methods, the total energy of the system is approximated by a sum of two terms [79]. The first, called the band structure energy, contains the quantum electronic effects; it is obtained by solving non self-consistent Kohn-Sham likeequation [80]. Here, the single electron wave functions are represented by linear combinations of atomic orbital. Hamiltonian and overlap matrix elements are evaluated in the framework of the two-centre approach of Slater-Koster [81]. The second is a short-ranged diatomic potential, which is fitted to ab-initio calculations or experimental measurements in order to reproduce a selected set of materials properties. Better accuracy and transferability is obtained using the self-consistent tight-binding methods [82]. In these methods, an additional term is added to the energy functional and adjusted through a self-consistent procedure. This is useful 
to take into account the charge transfer occurring in compound semiconductors like GaN. The Self-Consistent Charge Density Functional Tight-Binding (SCC-DFTB) [83-85] is a selfconsistent extension of the standard DFTB method [86]. The SCC-DFTB total energy was derived by expanding the Kohn-Sham energy functional to the second order with respect to the electronic density fluctuations around a given reference density [80, 83]. Besides the two usual energetic terms, i.e. the band structure energy and the short-ranger diatomic potential, a third term is introduced where a self-consistent procedure is incorporated at the level of Mulliken charges to account for charge transfer. This last term allowed to the SCC-DFTB technique to achieve accuracy comparable to that of ab-initio methods. Among the tightbinding methods, the SCC-DFTB method is one of most successful as applied to dislocations in $\mathrm{GaN}[2,78,87-91]$.

\subsection{Results of dislocation modelling in GaN}

From 1997, there has been a tremendous effort in the field of atomistic modelling to study dislocations in GaN. These studies attempt to provide some information about the core configuration of these dislocations and their effect on the electronic performance of the grown layers. Several kinds of dislocations have been investigated in wurtzite GaN; these were perfect or partial with edge, screw or mixed character, belonging to the prismatic or the basal plane.

\subsubsection{Perfect prismatic edge dislocation}

The prismatic edge dislocation $(\vec{b}=a / 3<11 \overline{2} 0>, l=<0001>)$ was the first dislocation to be investigated in wurtzite GaN [2]. This dislocation displays different core structures with stoichiometric configurations (8, 5/7 and 4 atom rings) (Fig.10) or non-stoichiometric configurations $\left(8-\mathrm{V}_{\mathrm{Ga}}\right.$ and $8-\mathrm{V}_{\mathrm{N}}$ atom rings). The core with 8 atom ring structure or full-core contains a column of three fold coordinated atoms whereas the 5/7 atom ring structure or 
Page 23 of 87

Philosophical Magazine \& Philosophical Magazine Letters

open-core contains wrong bonds $(\mathrm{Ga}-\mathrm{Ga}, \mathrm{N}-\mathrm{N})$. The core with 4 atom ring structure contains fully coordinated atoms establishing four in-plane bonds [20]. The non-stoichiometric $8-\mathrm{V}_{\mathrm{Ga}}$ or $8-\mathrm{V}_{\mathrm{N}}$ cores can be obtained from the core with 8 atom rings removing some of $\mathrm{Ga}$ or $\mathrm{N}$ atoms from the central column, while the complete removal of this column leads to the $5 / 7$ core $[2,14,21,66,88,92]$. Béré et al. have shown that the different cores $(8,5 / 7,4)$ are, in fact, obtained in a straightforward manner only by changing the origin of the displacement elastic field during the generation of the model [21]. In the framework of ab-initio and tightbinding (SCC-DFTB) calculations, Elsner et al. [2] reported that the core with 8 atom ring structure is more energetically favorable than that with 5/7 atom ring structure. However, all the subsequent calculations performed with different methods: empirical potentials -Modified Stillinger-Weber- [20, 76], tight-binding -SCC-DFTB- [88] and ab initio [23] suggest the opposite. In the case of the core with 4 atom ring structure, Chen et al. found it less energetically favorable than that with 8 atom ring structure [20].

(a)

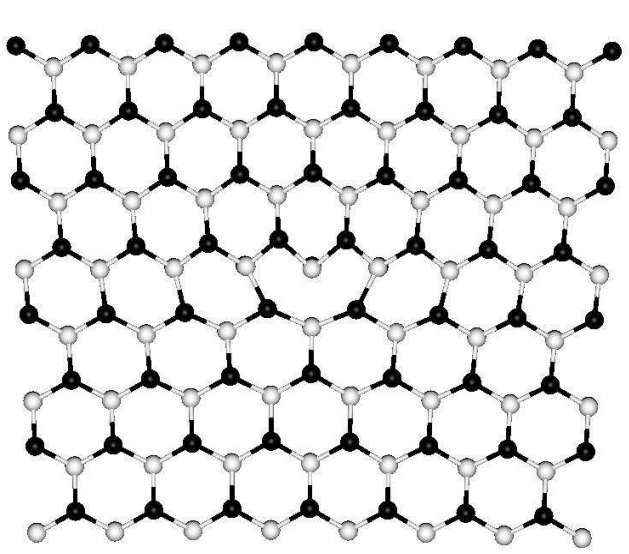

(b)

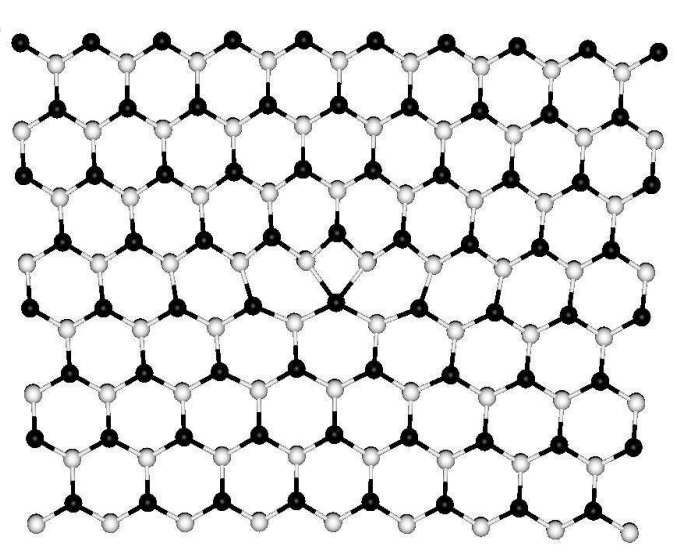

(c)

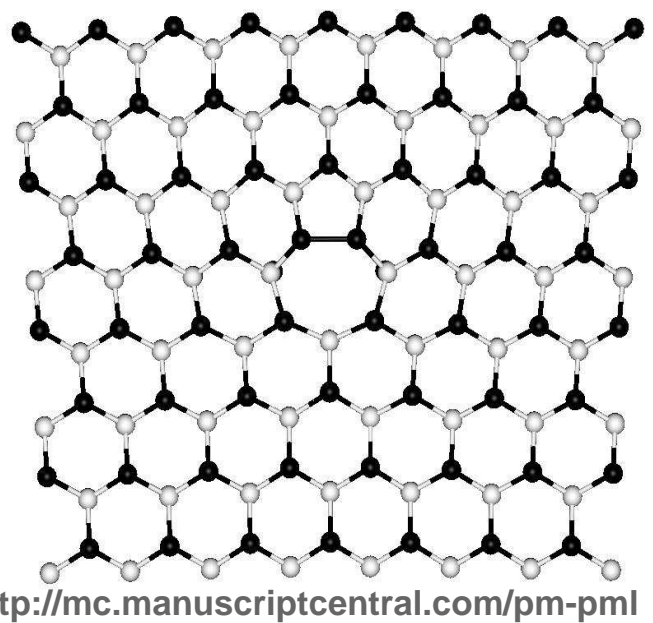

23 
Fig. 10: View along the [0001] direction of the atomic core configurations of the prismatic edge dislocation. a) 8 atom ring core, b) 4 atom ring core, c) 5/7 atoms ring core.

More recently, the core with 4 atom ring structure was reinvestigated by means of multiscale calculations: ab-initio, empirical potential and continuum elastic theory [23]. The authors showed that under a tensile strain, the 4 atom ring core becomes the most energetically favorable among the three cores of the stoichiometric edge threading dislocations.

Since the structure of tilt grain boundaries is described with a periodic array of the edge dislocation, they were investigated using our empirical potential. They were generated through rotations around the [0001] direction and it was then possible to show that the concept of structural units was valid for the hexagonal system [35]. In this instance, the building units for all grain boundaries were the 6 atom ring for the perfect lattice and the 5/7 atom core for the grain boundary dislocations (Fig. 11) [93].
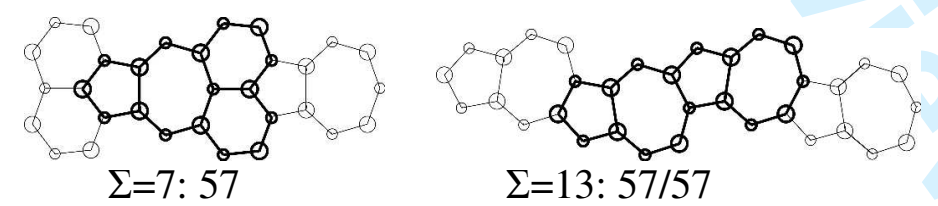
$\Sigma=13: 57 / 57$

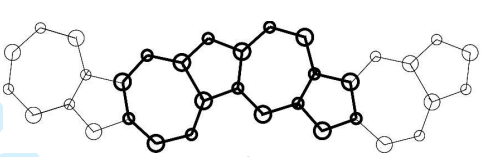

$\Sigma=13: 57^{+} 57^{-}$

Fig.11: Structural units for coincidence grain boundaries from rotations around [0001] based on the 5/7 atom ring core, the corresponding $\Sigma$ is indicated [93]

This was a good indication that the hexagonal system did not exhibit fundamentally different mechanisms for defect formation than the cubic, at least along high symmetry directions.

A set of calculations based respectively on ab-initio [66], tight-binding -SCC-DFTB[88] and Monte-Carlo [92] methods revealed that the formation energy of the prismatic edge dislocations with non-stoechiometric cores $\left(8-\mathrm{V}_{\mathrm{Ga}}\right.$ and $\left.8-\mathrm{V}_{\mathrm{N}}\right)$ strongly depends on both the growth conditions and the doping nature of the material. The $8-\mathrm{V}_{\mathrm{Ga}}$ core was shown to be 
more energetically favourable in n-type material grown under $\mathrm{N}$-rich conditions whereas the $8-\mathrm{V}_{\mathrm{N}}$ core is favourable in p-type material grown under Ga-rich conditions [66]. However, Lee et al. obtained favorable formation energies for cores with both 8 and 5/7 atom rings structures in material grown under Ga-rich conditions [88].

The electronic structure of the prismatic perfect edge dislocation with different core configurations has also been investigated extensively [2, 23, 66, 67, 88]. According Elsner et $a l$. the edge dislocation with 8 atom ring core structure is electrically inert and induces no gap states [2]. Therefore, it has undergone a relaxation of the three fold coordinated $\mathrm{Ga}(\mathrm{N})$ core atoms toward $\mathrm{sp}^{2}\left(\mathrm{p}^{3}\right)$ similar to the $(10 \overline{1} 0)$ surface, which leads the dimerization of the bonds along the dislocation line. However, several calculations performed on the same core configuration have revealed the presence of empty states at the half top of the band gap [22, $23,67,88]$. A quite similar electronic structure has been reported for the dislocation with 5/7 atom ring core structure (Fig.12) [23, 88].
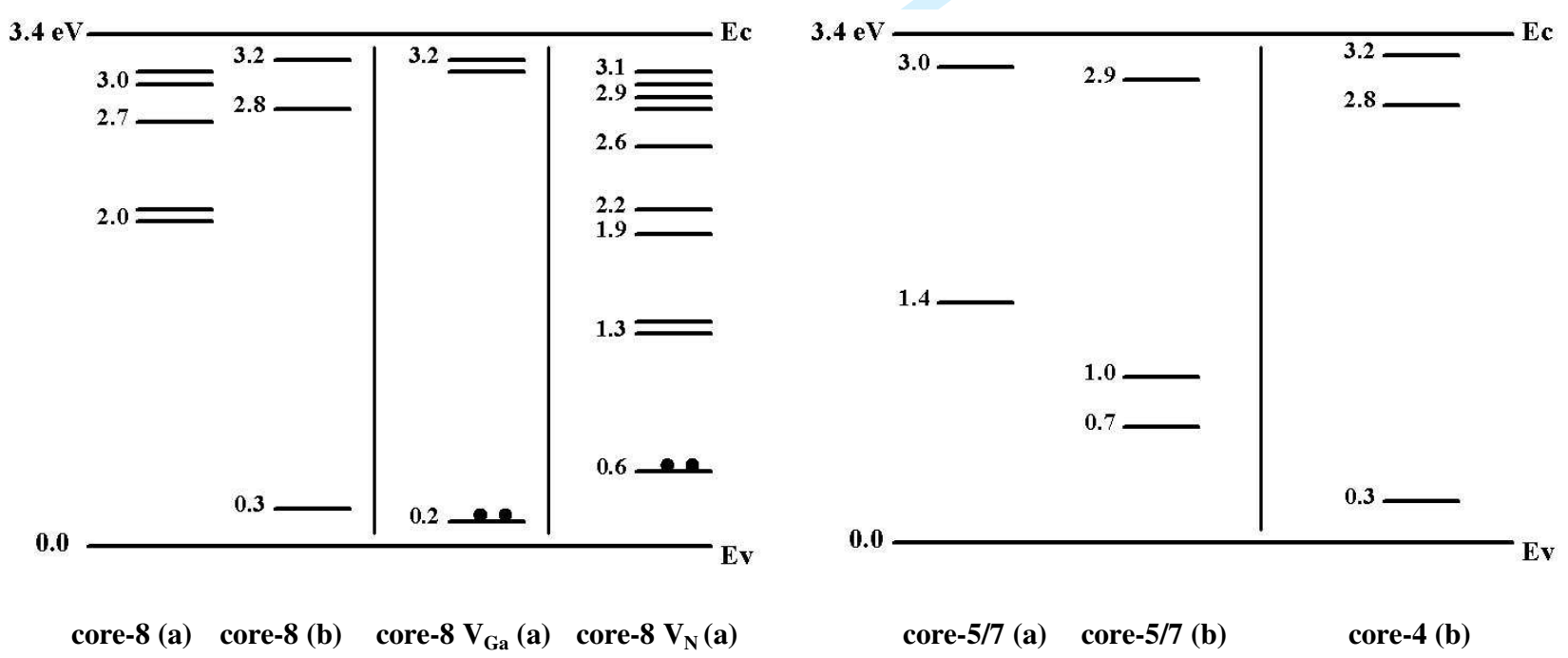

Fig.12: Schematic diagram of energy levels induced in the band gap by different cores of the prismatic edge dislocation: 8 atom ring core (core-8), 5/7 atom ring core (core-5/7) and the 4 atom ring core (core-4), dark balls indicate filled levels, (a) [88], (b) [23] 
For the 4 atom ring edge dislocation, an unexpected behavior has been reported recently: despite its fully coordinated core, it exhibits deep states in the band gap [23]. This was attributed to the large strain field surrounding the dislocation core which leads to the formation of bonding between Ga second neighbor atoms similar to metallic gallium. TightBinding calculations carried out on the non-stoichiometric structures have revealed for the 8$\mathrm{V}_{\mathrm{Ga}}$ core, the presence of both occupied states at $0.2 \mathrm{eV}$ from the VBM (Valence Band Maximum) and empty states just below the CBM (Conduction Band Minimum) [88]. The 8$\mathrm{V}_{\mathrm{N}}$ core has been shown to induce different states throughout the band gap; it may be expected to be an origin of yellow luminescence.

The electronic structures of the $(3 \overline{7} 40) \Sigma=37\left(\theta=9.3^{\circ}\right)$, $(3 \overline{5} 20) \Sigma=19\left(\theta=13.4^{\circ}\right)$ and $(1 \overline{3} 20) \Sigma=7\left(\theta=21.6^{\circ}\right)$ tilt grain boundaries were calculated. They were investigated by means of SCC-DFTB approach. Among the three possible atomic configurations of these boundaries, namely, the interfaces based on the $5 / 7$, the 4 and the, it was found that the structure based on the 8 atom ring core introduces deep states in the very center and the upper half of the band gap whereas the grain boundaries based on the 5/7 atom ring possess only states close to the conduction band (Fig.13) [90].

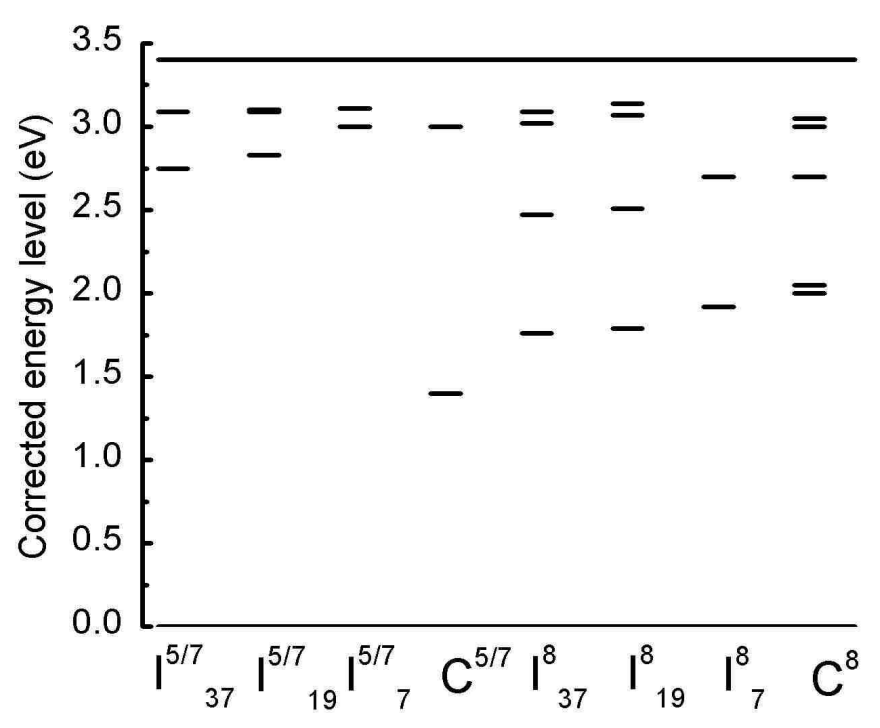




\subsubsection{Perfect prismatic screw dislocation}

The prismatic perfect screw dislocation $(\vec{b}=c<0001>, l=<0001>$,) is one of the most studied dislocation in wurtzite GaN. Several calculations have been carried out on their atomic and electronic core structures. Different core structures have been investigated: the full-core $[2,67,76,89]$, the open-core $[2,15]$ and non-stoichiometric configurations $[15,16]$ (Fig.14). In the first configuration, six stoichiometric atomic columns, in helical like symmetry arrangement along the direction [0001], form a hexagonal atomic core. By removing the latter, the open-core configuration is obtained. However, filling the core with different amounts of $\mathrm{Ga}$ and $\mathrm{N}$ atoms leads to non- stoichiometric configurations, where the Ga-filled and $\mathrm{N}$-filled cores represent the extreme limits. In their report, Elsner et al. have investigated prismatic screw dislocation with both full-core and open-core configurations [2]. The calculation of the line energy, in a cylinder of radius about $8 \AA$, gave $4.88 \mathrm{eV} / \AA$ for the full-core and $4.55 \mathrm{eV} / \AA$ for the open-core configuration. This was higher than their value of $2.19 \mathrm{eV} / \AA$ found for the full-core edge prismatic dislocation. Similar high line energy values 
were also reported by different authors for the screw full-core dislocation. This probably explains why the screw dislocations represent a small fraction with respect to the edge type in GaN epitaxial layers [15, 76, 89].

Recent ab initio calculations performed by Northrup $[15,16]$ revealed that screw dislocation with non-stoichiometric core configurations could be more energetically favourable than those with stoichiometric cores under some growth conditions. The Ga-filled core, obtained by removing all the $\mathrm{N}$ atoms from the hexagonal core, is predicted to be the most stable configuration under the Ga-rich conditions [15]. However, in N- rich conditions, a non-stoechiometric core structure with $\mathrm{Ga}(50 \%)$ and $\mathrm{N}(25 \%)$ atoms was found more energetically favourable [16].

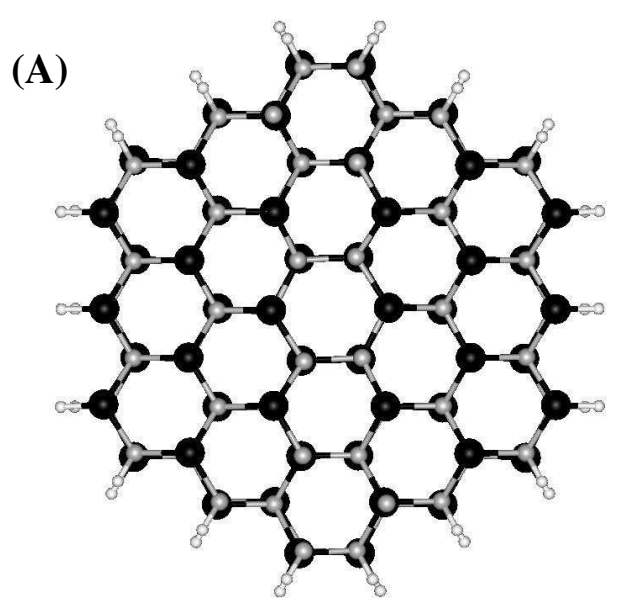

(C)

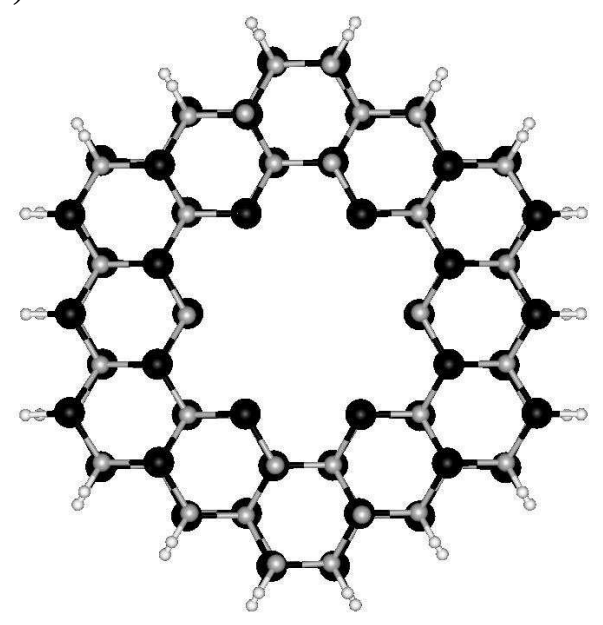

(B)

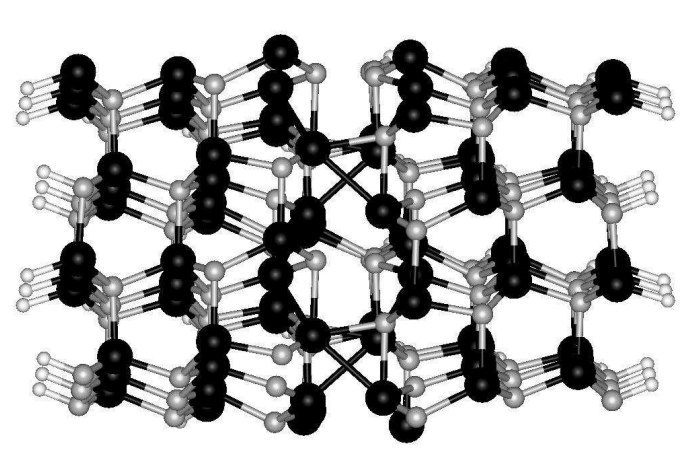

Fig.14: Atomic core structures of the prismatic screw dislocation:

A) View along the [0001] direction of the full-core structure. B) View along the $[11 \overline{2} 0]$ direction of the full-core structure. C) View along the [0001] direction of the open-core structure. 
In the full-core configuration, due to the presence of heavily distorted bonds, additional charge transfers should occur and gap states are induced. Belabbas et al. have identified an extra charge transfer of $0.12 \mathrm{e}$, besides that naturally occurring in $\mathrm{GaN}$ bulk material, from $\mathrm{Ga}$ to $\mathrm{N}$ atoms [89]. The electronic structure calculations performed on the full-core dislocation have revealed the presence in the band gap of shallow as well as deep states $[2,67]$. In the open-core dislocation, in order to lower the surface energy, the internal wall of the core relaxes like the $(10 \overline{1} 0)$ surface and then the threefold coordinated atoms $\mathrm{Ga}(\mathrm{N})$ adopt sp2 $\left(\mathrm{p}^{3}\right)$ hybridization. For this dislocation, only shallow states were reported and attributed to the distortion of the wall surface along the direction [0001] due to the Burgers vector [2]. For the non-stoichiometric dislocations, the Ga-filled core configuration was predicted to have a metallic like-behaviour as its induced states are highly dispersed in the entire band gap [15, $16,67]$. However, this behaviour is less pronounced in the most favourable core structure (Ga $50 \%, \mathrm{~N} 25 \%$ ) in N-rich conditions [16].

\subsubsection{Perfect basal edge and screw dislocations}

Up to date, there are only a few reports on the atomic core structures of the perfect basal edge $(\vec{b}=c<0001>, l=<11 \overline{2} 0>)$ and screw $(\vec{b}=a / 3<11 \overline{2} 0>, l=<11 \overline{2} 0>)$ dislocations [76, 78]. In the ELO (Epitaxial Lateral Overgrowth) process, the threading dislocations are bent [94]. Thus, a dislocation changes its line direction from $<0001>$ to $<11 \overline{2} 0>$ resulting in a new character, edge or screw. These basal dislocations can occur during the heteroepitaxial growth

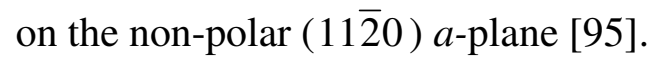

Recently, Belabbas et al. have investigated the atomic structure and the electronic activity of the basal edge dislocation in wurtzite $\mathrm{GaN}$ by combining empirical potentials (Modified Stillinger-Weber) and tight-binding methods (SCC-DFTB)[78]. For this dislocation 
four stable core configurations have been determined, and each corresponds to a particular position of the origin of the applied displacements field before relaxation (Fig.15a) [21]. G1 and G2 configurations belong to the glide set, S1 and S2 to the shuffle set. The G2 and S1 configurations display a 4/8 atom ring structure without wrong bonds (Fig.15c, d), whereas the G1 and S2 configurations which include wrong bonds, display a 5/8/5-atom rings structure (Fig.15b, e). The G1 and S2 configurations were already reported in the framework of empirical potentials [76].

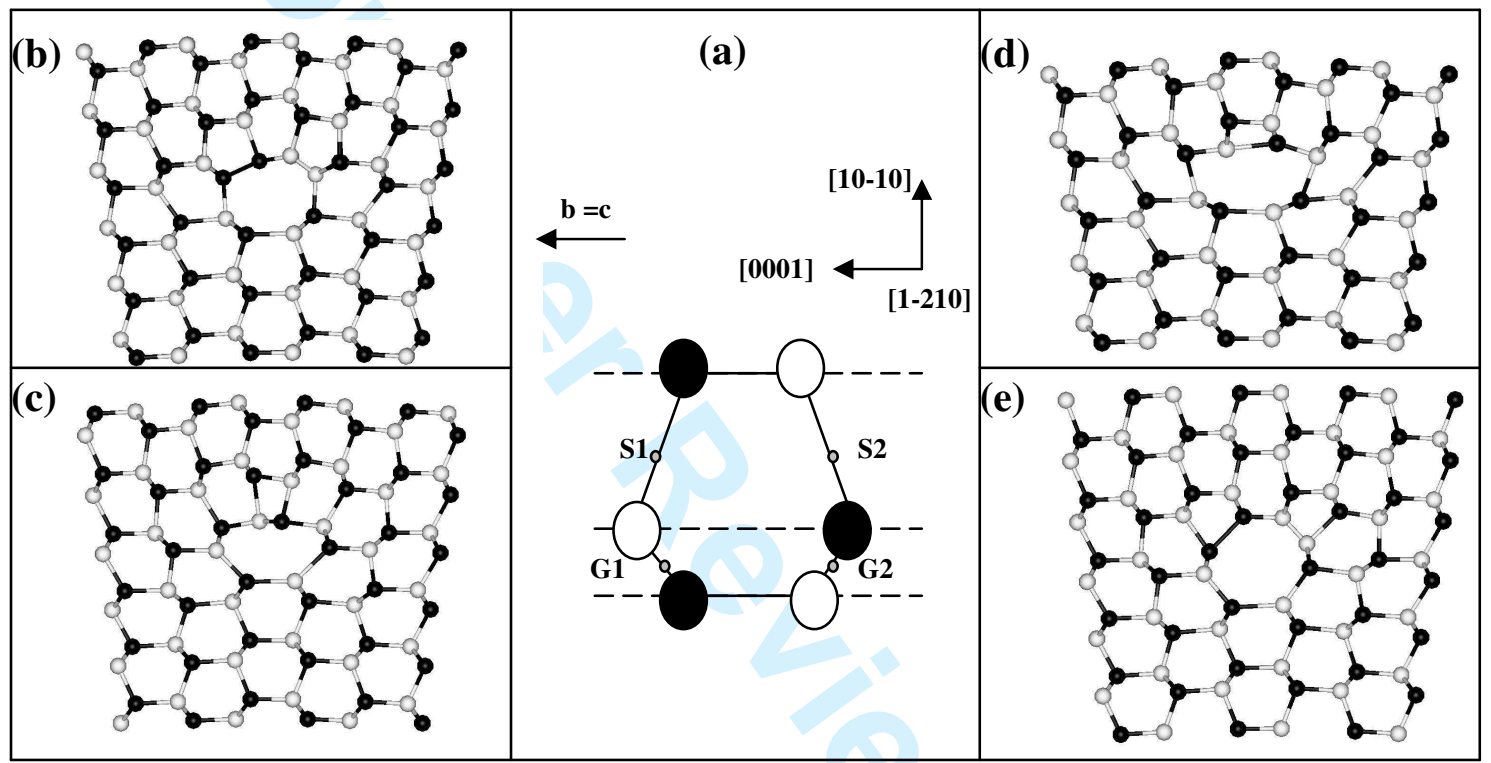

Fig.15: The four core configuration of the $\vec{c}$ edge basal dislocation: (a) Different positions of the origin of the applied displacements field, (b) G1 core configuration, (c) G2 core configuration, (d) S1 core configuration, (e) S2 core configuration. Black circles represent $\mathrm{Ga}$ atoms whereas white ones represent $\mathrm{N}$ atoms.

The SCC-DFTB energetic calculations for a core radius of $8.58 \AA$ gave for the four configurations: G1, G2, S1, S2, the following core energies: 5.24, 4.90, 5.73 and $4.44 \mathrm{eV} / \mathrm{A}$, respectively [78]. In both sets, the most favourable configuration is S2 with wrong bonds. In the glide set, the core without wrong bonds, G2, is energetically favoured. The electronic levels induced by each core configuration are presented in figure 16, where their positions in the band gap were corrected using the linear scaling method. 


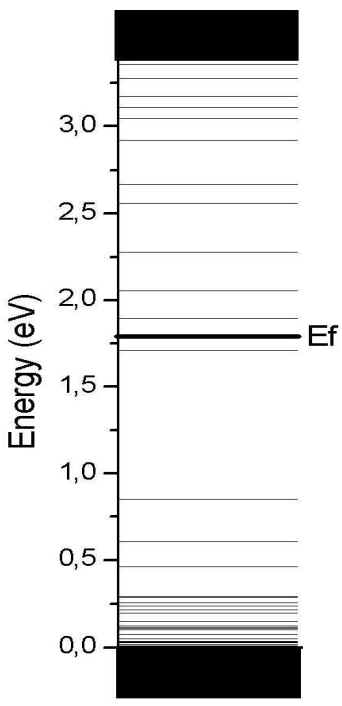

(a) G1 configuration

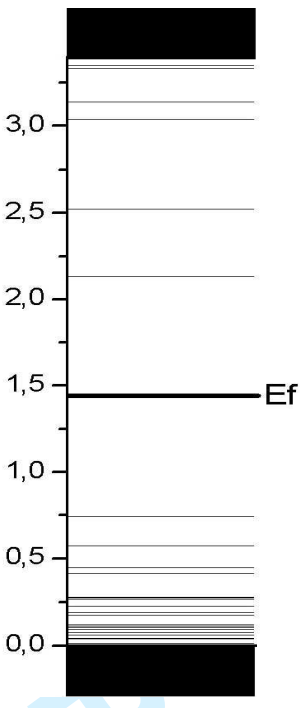

(b) G2 configuration

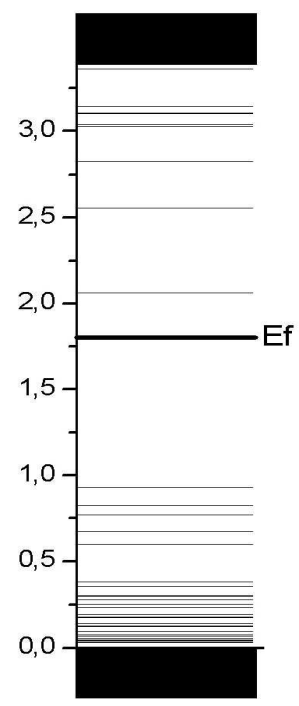

(c) S1 configuration

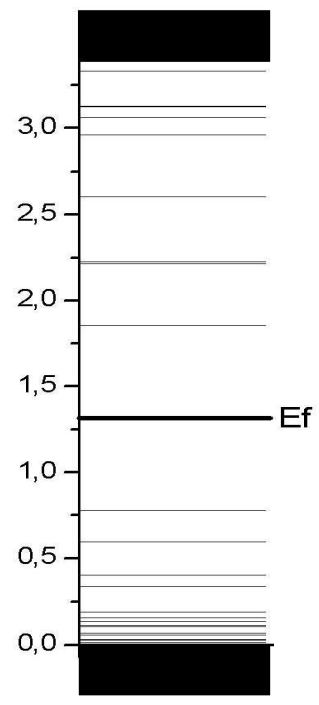

(d) S2 configuration

Fig. 16: Electronic gap states associated with different configurations of the basal edge dislocation. The zero has put on the top of the valence band maximum while the conduction band minimum is at $3.4 \mathrm{eV}$.

As can be seen, each core configuration induces deep as well as tail states. Filled states exist in the bottom half of the band gap, up to $1 \mathrm{eV}$ from the VBM. For all the configurations, unfilled deep states are present on the top half of the band gap, and are located mainly between 2 and $3 \mathrm{eV}$, from the VBM. In this area, the states induced by G1 are more spread than those of the other configurations. Due to the presence of the deep states, one could expect the basal edge dislocations to be at the origin of parasitic luminescence. However, it cannot be excluded that the G1 configuration may be involved in non-radiative recombination processes.

The atomic structure of the perfect basal screw dislocation has been only investigated by Béré et al., their study is based on a modified Stillinger-Weber potential [76]. Two stable core configurations were identified; one belongs to the glide set while the other belongs to the shuffle set. The screw dislocations were found to have a core diameter of $6.7 \AA$. The glide 
configuration has a line energy of $1.20 \mathrm{eV} / \mathrm{A}, 2 \%$ higher than that of the shuffle configuration.

\subsubsection{Perfect basal $60^{\circ}$ dislocation}

In zinc-blende GaN, Blumenau et al. have studied the atomic and electronic structures and the relative energies of the $60^{\circ}$ basal dislocation $(\vec{b}=\mathrm{a} / 2[1 \overline{1} 0], l=<01 \overline{1}>)$, with both glide and shuffle configurations (Fig.17) [22, 95].

(A)

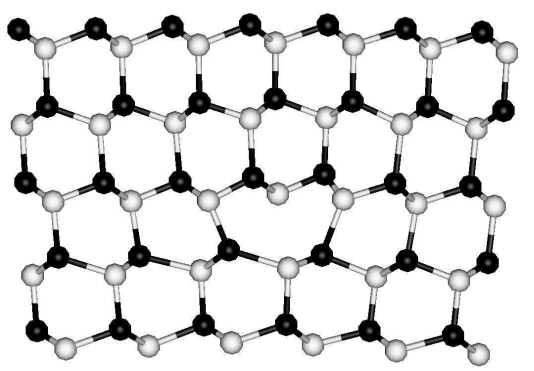

(C)

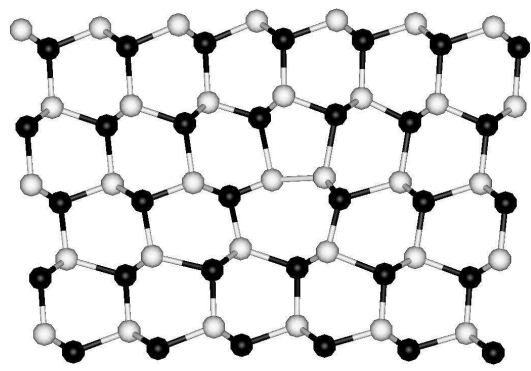

(B)

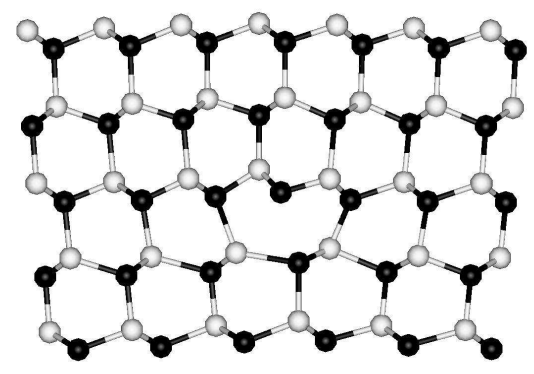

(D)

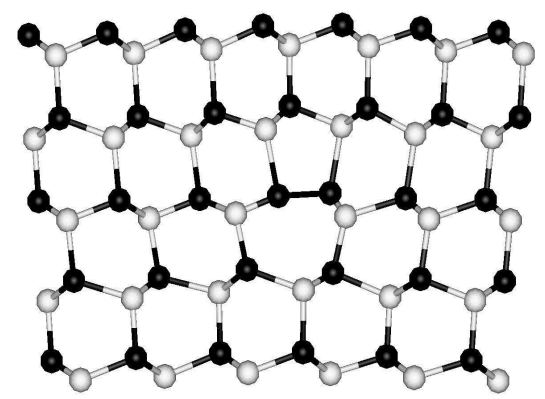

Fig. 17: The core configurations of the perfect $60^{\circ}$ dislocation in wurtzite GaN. (A) The $\alpha$ (N-terminated) shuffle configuration. (B) The $\beta$ (Ga-terminated) shuffle configuration. (C) The $\alpha$ (N-terminated) glide configuration. (D) The $\beta$ (Ga-terminated) glide configuration. Black balls represent gallium atoms and the white ones nitrogen atoms.

For the shuffle dislocations both the $\alpha$ (N-terminated) and $\beta$ (Ga-terminated) configurations were investigated. The $\alpha$ core shuffle dislocation was found to have an 8 atom rings like structure, with three fold coordinated atoms exhibiting a $\mathrm{sp}^{3}$ like hybridisation 


\subsubsection{Partial basal dislocations}

From their recent investigation, Savini et al. have reported the atomic and electronic structures of the $90^{\circ}$ Shockley partials, $(\vec{b}=a / 3[10 \overline{1} 0], l=<11 \overline{2} 0>)$, in wurtzite GaN (Fig.18) [68]. They used ab-initio calculations, implemented in the AIMPRO code, to model partial dislocations with $\alpha$ (N-terminated) and $\beta$ (Ga-terminated) cores in both supercells and cluster-supercell hybrids. By limiting their investigations to the glide configurations, they showed that the two partials can adopt both symmetric and asymmetric core reconstructions depending on the environment of the dislocation. The asymmetric reconstruction was found to 
be the most favourable and the energy difference between the two reconstructions was 83.2 meV for the $\beta$-core and $196.4 \mathrm{meV}$ for the $\alpha$-core. The calculated electronic structure
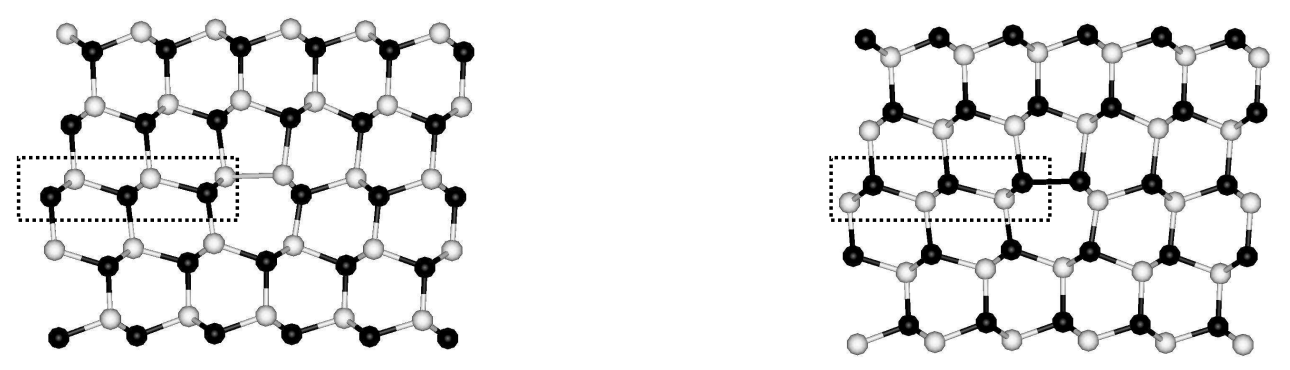

Fig.18: The core configurations of the $90^{\circ}$ Shockley partials. (A) The $\alpha$ (N-terminated) core configuration. (B) The $\beta$ (Ga-terminated) core configuration. The doted line indicates the intrinsic stacking fault. Black balls represent gallium atoms and the white ones nitrogen atoms.

exhibited mid gap states for both dislocations cores, and for the $\alpha$ core a deep acceptor level at $1.11 \mathrm{eV}$ and for the $\beta$ core a deep donor level at $0.87 \mathrm{eV}$, from the VBM, was pointed out. The first level is expected to give rise to a $2.3 \mathrm{eV}$ radiative transition which could contribute to the yellow luminescence [97], while the second one may be correlated to the $2.4 \mathrm{eV}$ absorption peak in agreement with energy loss spectroscopy measurements [98]. Based on Voronoi cell analysis of the charges density, it was shown that these partial dislocations present a charge polarization along [0001] with a negatively charged compressed region and a positively charged tensile region. Moreover, when these dislocations were brought together and in the presence of a high stress, a charge transfer was shown to occur from the $\beta$-core dislocation to the $\alpha$-core dislocation. It was concluded that such charges may be able to screen the carriers from recombination in the dislocation cores and explain why GaN devices may tolerate high dislocation densities.

In their empirical potentials investigation, Kioseoglou et al. have performed a systematic investigation of the atomic core structures and the corresponding energies of both edge 
$(\vec{b}=1 / 6[20 \overline{2} 3], l=[1 \overline{2} 10]$,$) and mixed (\vec{b}=1 / 6[2 \overline{2} 03], l=[1 \overline{2} 10])$ Frank-Shockley partials that bound the $I_{1}$ intrinsic basal stacking fault in wurtzite GaN [99]. For the edge and mixed partials, twelve stable configurations were found for each $\mathrm{Ga}$ and $\mathrm{N}$ core polarity, they consist of 5/7, 8 and 12 atom rings like structures. For the edge partials, the 5/7 atom rings structure was reported as the most energetically favourable with energies less than $0.76 \mathrm{eV} / \AA$. Whereas the 8 and 12 atom rings have energies ranging from 1.28 to $1.48 \mathrm{eV} / \AA$. For the mixed partials, both the 5/7 and 12 atom rings structures were most stable. Their core energies are larger than those of the edge dislocations and the energetically most favourable configurations have energies ranging from 0.81 to $1.05 \mathrm{eV} / \AA$. These partial dislocations should be electrically active since they present similar structures to those of the $60^{\circ}$ dislocations, already reported by Blumenau et al. in cubic GaN [95].

\section{Discussion}

The GaN layers are mostly grown on sapphire which exhibits a large mismatch, and the area in the vicinity of the interface with the substrate contains a lot of defects. As early as 1986 , low temperature buffer layers (AlN, GaN) were largely used in the growth of GaN resulting in high quality epitaxial layers [100]. In these layers deposited in the 500$600^{\circ} \mathrm{C}$ temperature range, it was shown that many basal stacking faults form and during the subsequent increase of the temperature for the active layer growth they may become the origin of the threading dislocations[47]. The connection of the stacking faults and the misfit dislocations has not yet been clearly demonstrated. However, the connection between the origin of the threading dislocations and the highly strained interfacial area with substrate was established [19]. This confirms more or less the rather simple picture of mosaic growth which was published as early as 1997 [101]. Following their climb at grain boundaries or from the SF reaction area in the buffer layer, the threading dislocations obtain their equilibrium 
structure as determined by the growth kinetics. i.e.: their line becomes mainly parallel to the [0001] growth direction. The three topological dislocations of the hexagonal lattice have been observed with a vast majority of $\vec{a}$ edge ones, as reported from the start. The mosaic growth of the layers allows the $\vec{a} 60^{\circ}$ interfacial dislocations to easily become pure edge along the $c$ axis forming low angle grain boundaries. The growth along the $c$ axis limits the formation of $\vec{a}+\vec{c}<10 \%$ dislocation and even more the $\vec{c} \sim 1 \%$ as observed experimentally.

As for the atomic structure, the investigations were first misled by the fact that, even if the first layers contained $10^{10} \mathrm{~cm}^{-2}$ dislocations, the LEDs emission was the brightest known in semiconductor optoelectronics.

The multiplicity of the atomic configurations was shown as early as 1998 with the coexistence of the 5/7 and 8 atom ring cores in good quality GaN layers types[13] and the occurrence of the additional 4 atoms core inside grain boundaries[18]. Of course, as it was demonstrated later on, the three configurations are just a consequence of the wurtzite structure, and the occurrence of each depends on the position of the dislocation line inside the unit cell [21]. Then, by using our empirical potential [75] along with ab initio calculations, Lymperakis at al. have carried out a multiscale analysis of this edge threading dislocation [23]. They convincingly demonstrated that, the 4 atom ring core which has been first reported in grain boundaries may be the most stable in strained layers [18].

At the initial stages of the growth, many stacking faults are involved and partial dislocations can be present. However, it is of interest to investigate their atomic structure. It is may be noticed that as stressed by Narayan et al. [47], they will quickly develop in threading dislocations and behave like the dislocations $\vec{a}, \vec{a}+\vec{c}$ and $\vec{c}$ of the hexagonal lattice.

The electronic structure of the threading dislocations has been extensively debated and now the situation is clearer. For the prismatic edge dislocation with full or open core, empty states are present in the half top of the band gap. Some discrepancies still remain about their 


\section{Conclusion}

In this paper, we presented and discussed our results dealing with the dislocations in GaN layers. The dislocations are strongly involved in degrading the performance of the devices. The atomic core structures of the perfect edge threading dislocations are based on only three types of atom rings, however, depending on the layer growth conditions, they can be stoichiometric or not. The perfect dislocations, threading and basal dislocations, have now been largely investigated unlike the partial dislocations. Their electrical activity is in good 
agreement with their atomic structure. A number of theoretical reports show that their electronic structures exhibit deep and shallow levels.

Acknowledgements: The authors acknowledge the support of the EU under contracts "IPAM" HPRN-CT-1999-00040, and "PARSEM" MRTN-CT-2004-005583. The computations were performed at "CRIHAN" (http://www.crihan.fr).

\section{References}

[1] S. Nakamura, T. Mukai and M. Senoh, Appl. Phys. Lett. 641687 (1994).

[2] J. Elsner, R. Jones, P. K. Sitch, V. D. Porezag, M. Elstner, Th. Frauenheim, M. I. Heggie, S. Öberg and P. R. Briddon, Phys. Rev. Lett., 793672 (1997).

[3] F.R. Chien, X.J. Ning, S. Stemmer, P. Pirouz, M.D. Bremser and R.F. Davis, Appl. Phys. Lett., 682678 (1996).

[4] X.H. Wu, L.M. Brown, D. Kapolnek, S. Keller, B. Keller, S.P. DenBaars, and J.S. Speck, J.Appl. Phys., 803228 (1996).

[5] A.F. Wright and J.S. Nelson, Phys.Rev.B, 517866 (1995).

[6] L. Sigiura, J. Appl. Phys., 811633 (1997).

[7] Y. Xin, S. J. Pennycoock, N.D. Browning, P.D. Nelist, S. Sivananthan, B. Beaumont, J. P. Faurie and P. Gibart, Mat. Res. Soc. Symp. Proc., 482781 (1998).

[8] P. Vermaut, P. Ruterana, G. Nouet and H. Morkoç, Phil. Mag. A, 75239 (1997).

[9] B. Daudin, J. L Rouviere. and M. Arlery, Appl.Phys.Lett., 692480 (1996).

[10] V. Potin, P. Vermaut, P. Ruterana and G. Nouet, J. Electron. Mater., 27266 (1998).

[11] N.G. Weimann, L.F. Eastman, D. Doppalapudi, H.M. Ng and T.D. Moustakas, J. Appl. Phys., 833656 (1998).

[12] S. Nakamura and G. Fasol, The Blue Laser Diodes (Springer, Heidelberg, 1997).

[13] P. Ruterana, V. Potin and G. Nouet, Mat. Res. Soc. Symp. Proc., 482459 (1998).

[14] A.F. Wright, Mat. Res. Soc. Symp. Proc., 482795 (1998). 
[15] J. E Northrup., Appl. Phys. Lett., 782288 (2001).

[16] J. E. Northrup, Phys. Rev. B, 66045204 (2002).

[17] I. Arslan and N.D. Browning, Phys.Rev. Lett., 91165501 (2003).

[18] V. Potin, P. Ruterana, G. Nouet, R.C. Pond and H. Morkoç, Phys. RevB., 615587 (2000).

[19] G.P. Dimitrakopulos, Ph. Komninou, J. Kioesoglou, Th. Kehagias, E. Sarigiannidou, G A.Georgakilas, G. Nouet and Th. Karakostas, Phys. Rev. B, 64245325 (2001).

[20] J. Chen, P. Ruterana and G.Nouet, Mater.Sci.Eng. B, 82117 (2001).

[21] A. Béré, J. Chen, P. Ruterana, A. Serra and G. Nouet, Compt. Mat. Sci., 24144 (2002).

[22] A.T. Blumenau, C.J. Fall, J. Elsner, R. Jones, M.I. Heggie and Th. Frauenheim, phys.stat.sol.(c), 01684 (2003).

[23] L. Lymperakis, J. Neugebauer, M. Albrecht, T. Remmele and H.P.Strunk, Phys. Rev. Lett., 93 196401(2004).

[24] D.B. Holt, J. Mater.Sci., 231131 (1988).

[25] T. Mattila and A. Zunger, J. Appl. Phys., 85160 (1999).

[26] J.P. Hirth and J. Lothe, Theory of dislocations (Wiley, New York, 1982).

[27] F.A. Ponce, D. Cherns, W.T. Young and J.W. Steeds, Appl. Phys. Lett., 69770 (1996).

[28] P. Gibart, B. Beaumont and P. Vennéguès, Nitride Semiconductors Handbook on Materials and Devices, edited by P. Ruterana, M.Albrecht, J. Neugebauer (Wiley-VCH, 2003), pp. 45.

[29] V. Potin, PhD Thesis, University of Caen (1999).

[30] P.A. Stadelmann, Ultramicroscopy, 21131 (1987).

[31] Y. Xin, S.J. Pennycoock, N.D. Browning, P.D. Nelist, S. Sivananthan, F. Omnès, B. Beaumont, J. P. Faurie and P. Gibart, Appl. Phys. Lett., 722680 (1998).

[32] V. Potin, P. Ruterana, A. Hairie and G. Nouet, Mat. Res. Soc. Symp. Proc., 482435 (1998).

[33] R.C. Pond, Dislocations in Solid, edited by F.R.N. Nabarro (North Holland, Amsterdam 1989) 8 pp.1.

[34] W. Bollmann, Crystal Defects and Crystalline Interface, (Springer, Berlin, 1970).

[35] J. Chen, P. Ruterana and G. Nouet, Phys. Rev. B, 67205210 (2003). 
[36] P. Ruterana, A.M. Sanchez and G. Nouet, Nitride Semiconductors Handbook on Materials and Devices, edited by P. Ruterana, M. Albrecht, J. Neugebauer (Wiley-VCH, 2003), pp. 379.

[37] H.M. Hobgood, D.L. Barrett, J.P. McHugh, R.C. Clarke, S. Sriram, A.A. Burk, J. Greggi, C.D. Brandt, R.H. Hopkins and W.J. Choyke, J. Cryst. Growth, 137181 (1994).

[38] Z. Liliental-Weber, Y. Chen, S. Ruvimov and J. Washburn, Phys. Rev. Lett., 792835 (1997).

[39] W. Qian, G. S. Rohrer, M. Skowrnonski, K. Doverspike, L. B. Rowland and D.K. Gaskill, Appl. Phys. Lett., 672284 (1995).

[40] P. Vennegues, B. Baumont, M. Vaille and P. Gibart, Appl. Phys. Lett., 702434 (1997).

[41] J.W.P. Hsu, M.J. Manfra, R.J. Molnar, B. Heying and J. S. Speck, Appl. Phys. Lett., 81 79 (2002).

[42] Z. Liliental-Weber, D. Zakharov, J. Jasisnski, J.Washburn, M.A. O'Keefe and H. Morkoç., Mat. Res. Soc. Sym. Proc., 743243 (2003).

[43] P. Kung, C.J. Sun, A. Saxler, H. Ohsato and M. Razeghi, J. Appl. Phys., 754515 (1994).

[44] S. Kaiser, H. Preis, W. Gebhart, O. Ambacher, H. Angerer, M. Stutzmann, A. Rosenauer and D. Gerthsen, Jpn. J. Appl. Phys., 3784 (1998).

[45] Th. Kehagias, Ph. Komninou, G. Nouet, P. Ruterana and Th. Karakostas, Phys. Rev.B, 64195329 (2001).

[46] L.A. Bendersky, D.V. Tsvetkov and Y.V. Melnik, J. Appl. Phys., 941676 (2003).

[47] V. Narayan, K. Lorenz., W. Kim and S. Mahajan, Appl. Phys. Lett. 781544 (2001).

[48] M. Albrecht, H.P.Strunk, J.L. Weyher, I. Grzegory, S. Porowsky and T.Wosinski, J. Appl.Phys., 922000 (2002).

[49] C. Stampfl and C.G. Van de Walle, Phys. Rev. B, 57 R15052 (1998).

[50] J.A. Chislom and P.D. Bristowe, J. Phys.: Condens. Matter., 115067 (1999).

[51] P. Ruterana and G. Nouet, phys.stat.sol.(b), 227177 (2001).

[52] I. Arslan, A. Bleloch, E.A. Stach and N.D. Browning, Phys.Rev. Lett., 94, 025504 (2005).

[53] J.W.P. Hsu, M.J. Manfra, S.N.G. Chu, C.H. Chen, L.N. Pfeiffer and R.J. Molnar, Appl.Phys.Lett., 783980 (2001). 
[54] T. Hino, S. Tomiya, T. Miyajima, K. Yanashima, S. Hashimoto, and M. Ikeda, Appl. Phys. Lett., 763421 (2000).

[55] E. G. Brazel, M. A.Chin, and V. Narayanamurti, Appl. Phys. Lett., 74, 2367 (1999).

[56] T. Sugahara, H.Sato, M. Hao, Y. Naoi, S.Kurai, S.Tottori, K. Yamashita, K. Nishino, L. Romano and S. Sakai, Jpn. J. Appl. Phys., 37 L398 (1998).

[57] T. Remmele, M. Albrecht, H.P. Strunk, A.T. Blumenau, M.I. Heggie, J. Elsner, F T. Frauenheim, H.P.D. Schenk and P. Gibart, Inst. Phys. Conf. , 169, 323 (2001).

[58] W.J. Tunstall, P.B. Hirsch. and J. Steeds, Phil. Mag. 9, 99 (1964).

[59] P. Hohenberg and W. Kohn, Phys. Rev. B, 136864 (1964).

[60] W. Kohn and L. Sham, Phys. Rev. A, 1401133 (1965).

[61] W.E. Pickett, Computer Physics Reports, 9115 (1989).

[62] G. Kresse and J. Furthmüller, Phys. Rev. B, 5411169 (1996).

[63] M. Bockstedte, A.Kley, J. Neugebauer and M. Scheffler, Comp.Phys.Commun., 107187 (1997).

[64] P.R. Briddon and R. Jones, phys.stat.sol.(b), 217131 (2000).

[65] J.M. Soler, E. Artacho, J. Gale, A. Garcia, J. Junquera, P. Ordejon and D. SanchezPortal, J. Phys.: Condens. Matter, 142745 (2002).

[66] A.F. Wright and U. Grossner, Appl. Phys. Lett., 732751 (1998).

[67] C.J. Fall, R. Jones, P. Briddon, A.T. Blumenau, Th. Frauenheim, M.I. Heggie, Phys. Rev. B, 65245304 (2002).

[68] G. Savini, M.I. Heggie, C.P. Ewels, N. Martsinovich, R. Jones and A.T. Blumenau, , Material Science Forum, 483-485 1057 (2005).

[69] M. Finnis, Forces in Condensed Matter (Oxford University Press, Oxford, 2003).

[70] P.N. Keating, Phys. Rev., 145637 (1966).

[71] G.A. Baraff, E.D. Kane and M.Schluter, Phys.Rev. B, 215665 (1980).

[72] F.H. Stillinger and T.A. Weber, Phys. Rev. B, 315262 (1985).

[73] J. Tersoff, Phys.Rev.B, 376991 (1988); Phys.Rev.B, 389902 (1988); Phys.Rev.Lett., 61 2879 (1988); Phys.Rev.B, 395566 (1989).

[74] M. Ichimura, phys. stat. sol. (a), 153431 (1996). 
[75] N. Aïchoune, V. Potin, P. Ruterana, A. Hairie, G. Nouet and E. Paumier, Comput. Mater. Sci., 17380 (2000).

[76] A. Béré and A. Serra, Phys. Rev.B, 66085330 (2002).

[77] J. Kioseoglou, H.M. Polatoglou, L. Lymperakis, G. Nouet and Ph. Komninou, Comp. Mater. Sci., 2743 (2003).

[78] I. Belabbas, J. Chen, M.A. Belkhir, P. Ruterana, and G. Nouet, paper presented at Symposium F of the E-MRS Fall Meeting, Warsaw, Poland, 2-5 September (2005).

[79] D.J. Chadi, Phys. Rev. Lett., 4379 (1979).

[80] W.M.C. Foulkes and R. Haydock, Phys. Rev. B, 3912520 (1989).

[81] J.C. Slater and G.F. Koster, Phys. Rev., 941498 (1954).

[82] A.P. Horsfield and A.M. Bratkovsky, J.Phys.:Condens. Matter., 12 R1 (2000).

[83] M. Elstner, D. Porezag, G. Jungnickel, J.Elsner, M. Haugk, T. Frauenheim, S.Suhai, and G. Geifert, Phys. Rev. B 587260 (1998).

[84] T. Frauenheim, G. Seifert, M. Elstner, Z. Hajnal, G. Jungnickel, D. Porezag, S. Suhai, and R. Scholz, phys. stat. sol.( b), 21741 (2000).

[85] T. Frauenheim, G. Seifert, M. Elstner, T. Niehaus, C. Kohler, M. Amkreutz, M. Sternberg, Z. Hajnal, A. Di Carlo and S. Suhai, J. Phys.: Condens. Matter, 143015 (2002).

[86] D.Porezag, Th. Frauenheim, and Th. Köhler, Phys. Rev. B 5112947 (1995).

[87] M. Haugk, J. Elsner, T. Frauenheim, T.E.M. Staab, C.D. Latham, R. Jones, H. S.Leipner, T. Heine, G. Seifert and M. Sternberg, phys. stat. sol.(b), 217473 (2000).

[88] S.M. Lee, M.A.Belkhir, X.Y. Zhu, Y.H. Lee, Y.G. Hwang and Th. Frauenheim, Phys. Rev. B, 6116033 (2000).

[89] I.Belabbas, M.A. Belkhir, Y.H. Lee, A. Béré, P. Ruterana, J. Chen, and G. Nouet, phys. stat. sol. (c), 22492 (2005).

[90] A. Béré, P. Ruterana, G. Nouet, A.T. Blumenau, S. Sanna, T. Frauenheim, J.Chen and J. Koulidiati, Phys. Rev.B, 71125211 (2005).

[91] A. Béré, J. Chen, G. Nouet, P. Ruterana and J. Koulidiati, phys.stat.sol.(c), 22496 (2005).

[92] K. Leung, A.F. Wright and E.B. Stechel, Appl. Phys. Lett., 742495 (1999). 
[93] J. Chen, P. Ruterana and G. Nouet, presented at Symposium F of the E-MRS Fall Meeting, Warsaw, Poland, 2-5 September (2005).

[94] P. Vennegues, B. Beaumont, V. Bousquet, M. Vaille and P. Gibart, J. Appl. Phys., 87 4175 (2000).

[95] B.A. Haskell, F. Wu, S. Matsuda, M.D. Craven, P.T. Fini, S. Den Baars, J.S. Speck and S. Nakamura, Appl. Phys. Lett., 831554 (2003).

[96] D.J. As, A. Richter, J.Busch, B. Schottker, M. Lubbers, J. Mimkes, D. Schikora, K. Lischka, W. Kriegseis, W. Burkhardt and B.K. Meyer, MRS Internet J. Nitride Semicond. Res., 5S1 W3.81 (2000).

[97] X.G. Qiu, Y.Segawa, Q.K. Xue, Q.Z. Xue and T. Sakurai, Appl. Phys. Lett., 771316 (2000).

[98] A. Gutierrez-Sosa, U. Bangert, A.J.Harvey, C.J. Fall, R. Jones, P.R. Briddon and M.I. Heggie, Phys. Rev. B, 6635302 (2002).

[99] J. Kioseoglou, G.P. Dimitrakopulos, Ph. Komninou and Th.Karakostas, Phys. Rev. B, 70 035309 (2004).

[100] H. Amano, N. Sawaki, I. Akasaki and Y. Toyoda, Appl. Phys. Lett., 48353 (1986).

[101] F.A. Ponce and D.P. Bour, Nature, 386, 351 (1997). 
The atomic and electronic structure of dislocations in $\mathbf{G a}$ based nitride semiconductors

\begin{tabular}{|r|l|}
\hline Journal: & Philosophical Magazine \& Philosophical Magazine Letters \\
\hline Manuscript ID: & TPHM-05-Sep-0420.R2 \\
\hline Journal Selection: & Philosophical Magazine \\
\hline Date Submitted by the & n/a \\
\hline Complete List of Authors: & $\begin{array}{l}\text { BELABBAS, Imad; ENSICAEN, SIFCOM } \\
\text { CHEN, Jun; IUT, LRPMN } \\
\text { RUTERANA, Pierre; ENSICAEN, SIFCOM } \\
\text { NOUET, Gérard; ENSICAEN, SIFCOM }\end{array}$ \\
\hline Keywords: & GaN, atomic structure, dislocations, electronic structure \\
\hline Keywords (user supplied): & \multicolumn{2}{|c}{} \\
\hline \multicolumn{2}{|c}{} \\
\hline
\end{tabular}

\section{powered by ScholarOne Manuscript Central ${ }^{\text {MN }}$}




\title{
The atomic and electronic structure of dislocations in Ga based nitride semiconductors
}

\author{
I. Belabbas ${ }^{1,3}$, P.Ruterana ${ }^{1}$, J. Chen $^{2}$, G. Nouet $^{1}$ \\ ${ }^{1}$ Laboratoire Structure des Interfaces et Fonctionnalité des Couches Minces, UMR CNRS \\ 6176, Ecole Nationale Supérieure d'Ingénieurs de Caen, 6 Bld du Maréchal Juin, 14050 \\ Caen cedex, France. \\ ${ }^{2}$ Laboratoire de Recherche sur les Propriétés des Matériaux Nouveaux, IUT d'Alençon, \\ 61250 Damigny, France \\ ${ }^{3}$ Groupe de Physique du Solide, Laboratoire de Physique Théorique, Université A.Mira, \\ Béjaia, 06000, Algérie.
}

\begin{abstract}
The atomic and electronic properties of dislocations in III-N semiconductor layers, especially $\mathrm{GaN}$ are presented. The atomic structure of the $\vec{a}$ edge threading dislocation is now well established with three different cores ( 8 or full core, 5/7 or open core and 4 atom ring). The use of atomistic simulations has confirmed these atomic structures and has given a good understanding of the electronic structure of the screw dislocation. Partial dislocations which are mostly confined in the area close to the substrate are now also being investigated. It is becoming clear that the electrical activity of all these defects is dependent on the layer quality, which is governed by the growth conditions.
\end{abstract}




\section{Introduction}

During the last decade, III-V nitride semiconductors have taken an important part in optoelectronic devices since the first commercial light emitting diode (LED) in 1993 fabricated from metal organic vapour phase epitaxy (MOVPE) GaN/InGaN heterostructures containing a very large number of threading dislocations $\left(10^{10} \mathrm{~cm}^{-2}\right)[1]$. These LEDs were reliable although the dislocation density was higher compared to LEDs based on GaAs which emitted in the red region with defect densities below $10^{2}-10^{3} \mathrm{~cm}^{-2}$. The nature and properties of the dislocations in GaN needed to be understood, and the first reports tended to show that these dislocations should have at least a particular atomic structure which allows them to be non-active in the electronic processes [2]. By conventional transmission electron microscopy (TEM), it was shown that the only dislocations which formed in wurtzite GaN corresponded to the dislocations of the hexagonal lattice with the Burgers vectors $\vec{b}=\vec{a}, \vec{a}+\vec{c}$ and $\vec{c}$ [34]. The non activity of threading dislocations was assumed in the first theoretical report on the atomic structure of the $\vec{a}$ edge threading dislocation; it was proposed that the edge threading dislocation should have either a core based on an 8 atom ring with the dangling bonds reconstructed along the [0001] line or an empty core [5]. At the same time, a theoretical investigation of the motion of the prismatic threading edge dislocations and of the basal screw dislocations in $\mathrm{GaN}$ concluded that the mobility of the dislocations in GaN was very small at room temperature, a factor of approximately $10^{-10}$ to $10^{-16}$ with respect to GaAs, which could help to explain the remarkable reliability of GaN based LED [6].

A confirmation of the atomic structure came from the first report of Z-contrast results using high resolution scanning transmission microscopy [7], it was suggested that the $\vec{a}$ edge threading dislocation in GaN should have the reconstructed 8 atom core structure. In the meantime, extensive experimental work had been done on other extended defects which 
seemed to be typical for the GaN; these were prismatic stacking faults [8] and inversion domains [9-10].

At the same time, the electrical properties investigations were also analysed showing that GaN nitride layers had quite low carrier mobility due to the presence of the large densities of dislocations [11]. In fact, it was becoming clear that in order to fabricate devices such as lasers in which there is propagation of carriers in the basal plane, the density of threading dislocations needed to be decreased. As a consequence, the first 10000 hours life time laser diode was fabricated in a GaN layer grown using epitaxial lateral overgrowth and having an average threading dislocation density $<10^{8} \mathrm{~cm}^{-2}$ [12]. So, it was necessary to carry out extensive work on the dislocations in order to explain their true behaviour in GaN layers. Simultaneously to the Z-contrast report, our high resolution transmission electron microscopy (HRTEM) investigation showed that the edge threading dislocation had more than the 8 atom core configuration [13]. Mainly, in agreement with the theoretical work of Wright [14] as the open core configuration could not be distinguished with the proposed $5 / 7$ atom structure in HRTEM observations.

During the last few years, a large number of investigations have been carried out on the structure and properties of all types of dislocations in $\mathrm{GaN}$ as well as the possible effect of deviation from stoichiometric composition [15-16] and impurities [17]. Latterly, this effort has also been focused on the partial dislocations and their connections to other extended defects [18-19].

In this paper, we discuss the numerous results that have been reported along with our contribution on experimental HRTEM and theoretical investigation of these defects. Typically, our work has allowed to determine the multiple configuration of the threading edge dislocation and to clarify the crystallographic relationships between them [20-21]. The related study of electronic properties is a topic of high interest and different methods have been used 
to calculate the energy and electronic structure of defects in semiconductors. Empirical potentials still provide guidance in the most complex situations where large sets of atoms need to be used, but they cannot give any information on the electronic properties due to their analytical characteristics chosen to fit with elastic or phonon properties. The tight-binding theory has addressed an important field of computational materials science; it is a reasonable approach for the calculation of atomic and electronic structures in covalently bonded materials. In this vein, the density functional theory (DFT)-based tight-binding (DFTB) method operating in a self-consistent charge mode has offered a robust and transferable method to model a semiconductor crystal on the atomic scale [22]. Indeed, in the case of dislocations in GaN, insight has been gained in the most recent approaches which are multiscale, going from the empirical potentials to deal with large sets of atoms, to ab-initio modelling of the core of area [23]. In GaN, many configurations of the dislocations have now been investigated using these methods, and their atomic and electronic structures are reported on and discussed here. In a first part, we describe the crystallography of the structure and mainly of the dislocations. The following part deals with the experimental analysis of prismatic and basal dislocations, and their electrical behaviour. In the end, we discuss the atomistic simulation of the dislocation cores, and the calculations of their electronic structures.

\section{Crystallographic data}

\subsection{Structure}

Gallium nitride, GaN, crystallises in the cubic system with the zincblende form (sphalerite) or in the hexagonal system (wurtzite) [24]. For both forms, atoms have a tetrahedral coordination and the ideal angle between bonds is $109^{\circ} 5$. The sphalerite structure 
(a)

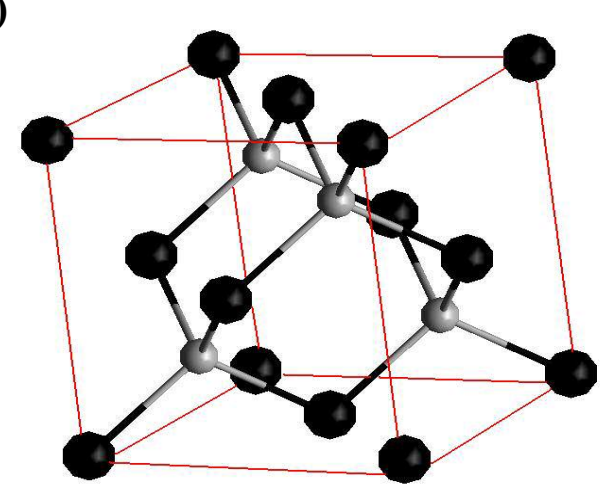

(b)

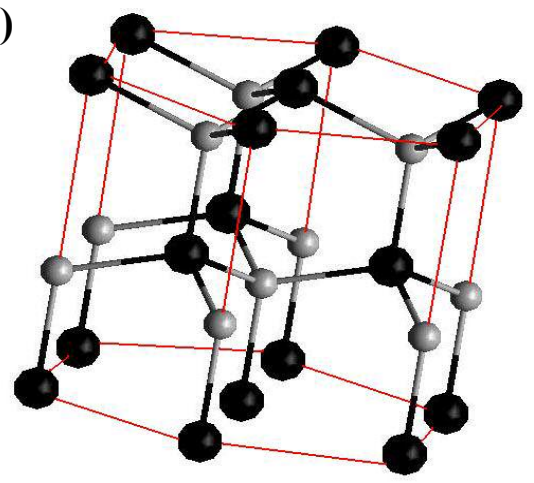

Fig.1: Structures of zinc blende and wurtzite crystals. a) zinc blende unit cell. b) wurtzite unit cell ( $\mathrm{Ga}$ : large black spheres, N: small grey spheres)

The zinc blende structure has only one type of first neighbour distance, which means that the four bond lengths in a tetrahedron are equal. This is also valid for a wurtzite structure with the ideal values $c / a=1.633$ and $u=3 / 8$. Otherwise, the bond parallel to the $c$ axis is 
different, within less than $1 \%$ for the III-V nitrides. For the second nearest neighbours, the zinc blende structure has only one type of bond lengths (12 bonds). The wurtzite structure presents three types of second neighbours, and for the ideal values, the difference between the shortest and the longest bonds is $13 \%$ [25].

\subsection{Dislocations}

In the frame of the linear elasticity theory of isotropic materials, the displacement field of the dislocation can be calculated as well as the induced stresses, except in the vicinity of the dislocation line or dislocation core where both stress and strain become infinite and this analysis breaks down. The strain energy of an infinite straight dislocation in a perfect crystal is calculated per unit length when the dislocation is contained in a cylinder of radius R. For a screw or an edge dislocation, this stored elastic energy is proportional to the square of the Burgers vector, $b^{2}$. The ratio of their energies is equal to the factor $(1-v), v$ is the Poisson's ratio, and the energy of an edge dislocation is larger than that of a screw dislocation. The total energy of the dislocation also includes the energy stored in the dislocation core. In the linear elastic theory, the displacements vary slowly; this assumption breaks down in the core region and atomistic calculations become necessary to calculate the corresponding energy.

Since the elastic energy of a dislocation is proportional to the square of the Burgers vector, this value of $b^{2}$ may be used to define the stability of a dislocation by applying the Frank criterion to the splitting of a dislocation with Burgers vector $\vec{b}_{1}$ into dislocations $\vec{b}_{2}$ and $\vec{b}_{3}$. The splitting takes place only if: $\left(\mathrm{b}_{1}\right)^{2}>\left(\mathrm{b}_{2}\right)^{2}+\left(\mathrm{b}_{3}\right)^{2}$. Applying this criterion, the possible Burgers vectors are only the primitive translations of the lattice. As a consequence, the Burgers vectors of the perfect dislocations in the zinc blende and wurtzite structures are identical to those of diamond or hexagonal close-packed material, respectively [26] (Table 1). 


\begin{tabular}{|c|c|c|c|}
\hline \multicolumn{2}{|c|}{ Zinc blende } & \multicolumn{2}{c|}{ Wurtzite } \\
\hline Burgers vector $(\vec{b})$ & $\left(\mathrm{b}^{2}\right)$ & Burgers vector $(\vec{b})$ & $\left(\mathrm{b}^{2}\right)$ \\
\hline $1 / 2<110>$ & $\mathrm{a}^{2} / 2$ & $1 / 3<1 \overline{120}>$ & $\mathrm{a}^{2}$ \\
\hline$<100>$ & $\mathrm{a}^{2}$ & $<0001>$ & $\mathrm{c}^{2}$ \\
\hline & & $1 / 3<1 \overline{12} 3>$ & $\mathrm{a}^{2}+\mathrm{c}^{2}$ \\
\hline
\end{tabular}

Table 1: Perfect dislocations in zinc blende and wurtzite structures: Burgers vector $(\vec{b})$ and energy $\left(b^{2}\right)$.

By considering the link between the crystallographic structure of crystals and the structure of dislocations, dislocations with Burgers vectors not belonging to lattice translations must be present. Such dislocations are defined as partial dislocations and the area connecting two partial dislocations is faulted (Table 2). As a first approximation, the application of the Frank criterion shows that the dissociation of a perfect dislocation into partials may take place.

\begin{tabular}{|c|c|c|c|c|c|}
\hline \multicolumn{3}{|c}{ Zincblende } & \multicolumn{3}{c|}{ Wurtzite } \\
\hline Type & Burgers vector $(\vec{b})$ & $\left(\mathrm{b}^{2}\right)$ & Type & Burgers vector $(\vec{b})$ & $\left(\mathrm{b}^{2}\right)$ \\
\hline Shockley & $1 / 6<112>$ & $\mathrm{a}^{2} / 6$ & Shockley & $1 / 3<10 \overline{1} 0>$ & $\mathrm{a}^{2} / 3$ \\
\hline Frank & $1 / 3<111>$ & $\mathrm{a}^{2} / 3$ & Frank & $1 / 2<0001>$ & $\mathrm{c}^{2} / 4$ \\
\hline \multirow{3}{*}{} & $1 / 6<110>$ & $\mathrm{a}^{2} / 18$ & Frank-Shockley & $1 / 6<20 \overline{2} 3>$ & $\mathrm{a}^{2} / 3+\mathrm{c}^{2} / 4$ \\
\cline { 2 - 3 } & $1 / 3<001>$ & $\mathrm{a}^{2} / 9$ & & \\
\cline { 2 - 3 } & $1 / 3<110>$ & $2 \mathrm{a}^{2} / 9$ & & & \\
\cline { 2 - 3 } & $1 / 6<301>$ & $5 \mathrm{a}^{2} / 18$ & & & \\
\hline
\end{tabular}

Table 2: Partial dislocations in zinc blende and wurtzite structures: Burgers vector $(\vec{b})$ and energy $\left(b^{2}\right)$.

For the nitrides, the perfect dislocations that have been characterised are the prismatic dislocation or edge dislocation $\vec{b}=1 / 3<11 \overline{2} 0>, l=<0001>$ with three different atomic cores and the screw dislocation $\vec{b}=<0001>, l=<0001>$ with two atomic cores (Fig.2).
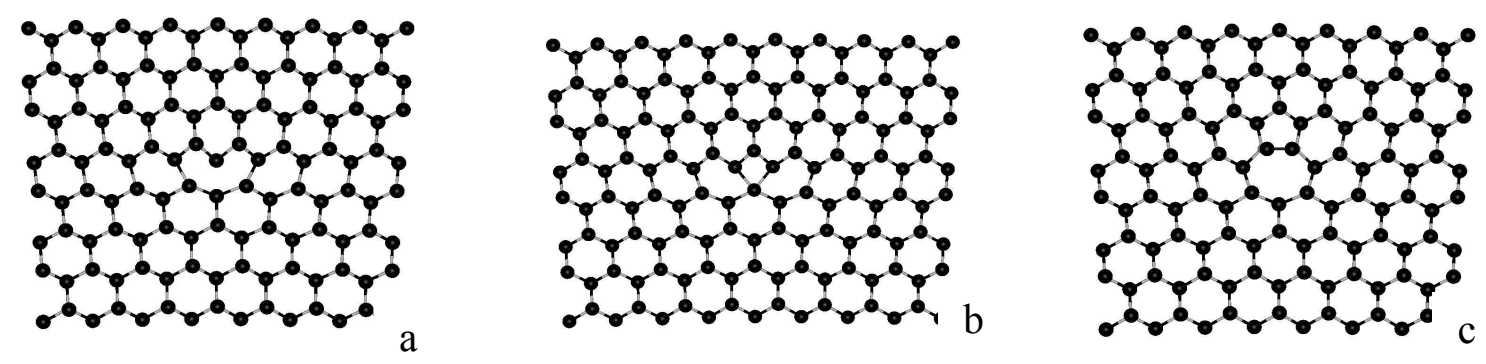

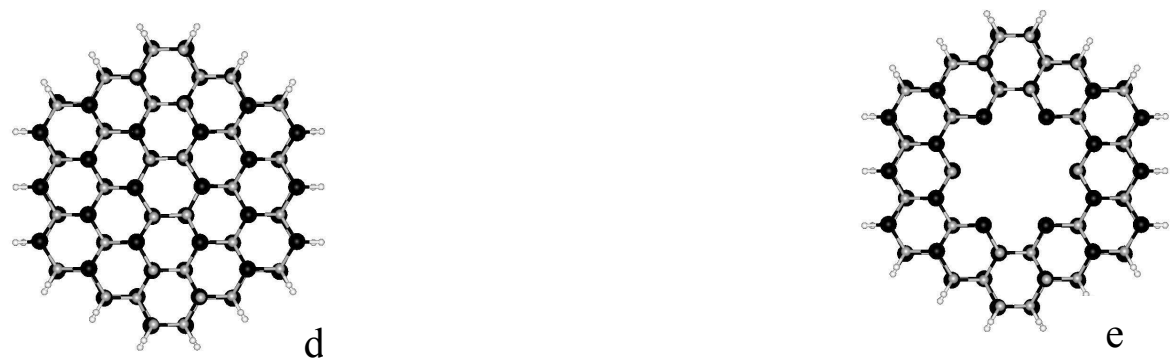

Fig.2: Prismatic dislocation or edge dislocation $\vec{b}=1 / 3<11 \overline{2} 0>, l=<0001>$ a: Full core or 8 atom ring, b: 4 atom ring, c: Open core or 5/7 atom ring and screw dislocation $\vec{b}=<0001>, l=<0001>$ d: Full core, e: Open core

In the basal plane, they may be $60^{\circ}$ (the angle between the line direction and the Burgers vector is $\left.60^{\circ}\right), \quad \vec{b}=[11 \overline{2} 0], l=[2 \overline{1} \overline{1} 0]$ or screw dislocations, $\vec{b}=[11 \overline{2} 0], l=$ $[11 \overline{2} 0]$

\section{Experimental observations}

\subsection{Prismatic dislocations}

The heteroepitaxially grown GaN layers on (0001) sapphire substrates whatever the growth process, e.g. molecular beam epitaxy (MBE) or metal organic chemical vapour deposition (MOCVD), are characterised by high densities of threading dislocations running across the layer from the layer/substrate interface to the top surface with their line mainly parallel to the $<0001>$ growth direction [27]. This density is in the order of $10^{9} \mathrm{~cm}^{-2}$ in conventional growth and can go up to $10^{11} \mathrm{~cm}^{-2}$ whatever the growth method; pure edge dislocation $(\vec{b}=1 / 3<1 \overline{2} 0>, l=<0001>)$ and mixed dislocation $(\vec{b}=1 / 3<11 \overline{2} 3>, l=<0001>)$ concentrations may be comparable and the fraction of pure screw dislocations $(\vec{b}=<0001>, l$ $=<0001>$ ) is usually low: 0.1 to $1 \%$ (Fig. 3) [28]. 

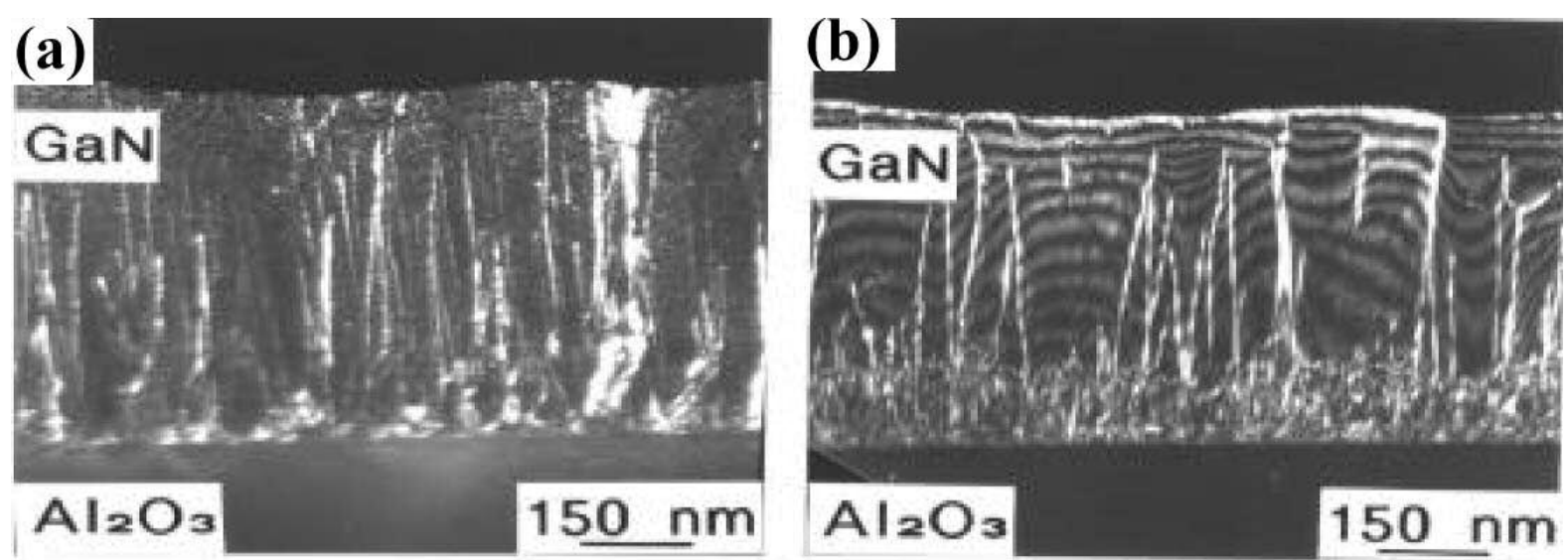

Fig. 3: Typical threading dislocations in MBE GaN layers grown on sapphire:

$$
\text { dark field a) } \mathrm{g}=1 \overline{12} 0 \text { b) } \mathrm{g}=0002 \text { [29] }
$$

\subsubsection{Edge or mixed threading dislocations}

The first observations of these threading dislocations by HRTEM showed that in good quality $\mathrm{GaN}$ layers grown by $\mathrm{MBE}$ at $800^{\circ} \mathrm{C}$ on (0001) sapphire, two atomic configurations coexisted: the 8 atom core and a 5/7 atom core (Fig.4) [13].

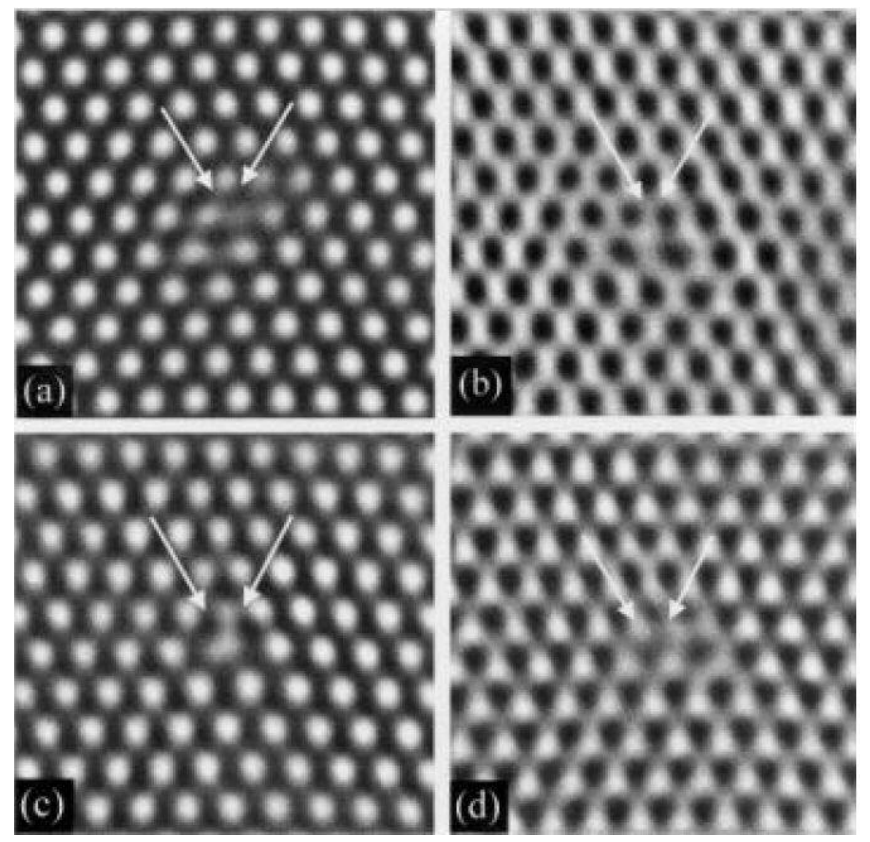

Fig. 4: HREM images along $<0001>$ of the $1 / 3<1 \overline{12} 0>$ edge dislocation: 8 atom ring ( $a$ and $b)$, $5 / 7$ atom ring ( $c$ and $d$ ), (defocus values, a and c:-24nm, b and d:-54nm) [18]

Later, in the investigation of low and high angle grain boundaries [18], it was shown that a configuration with a 4 atom core is also present in the GaN layers (Fig.5). The identification of the atom cores is based on the analysis of experimental and simulated contrasts of the 
images [30]. This was shown to be in agreement with the atomistic calculations of stoichiometric configurations [20].

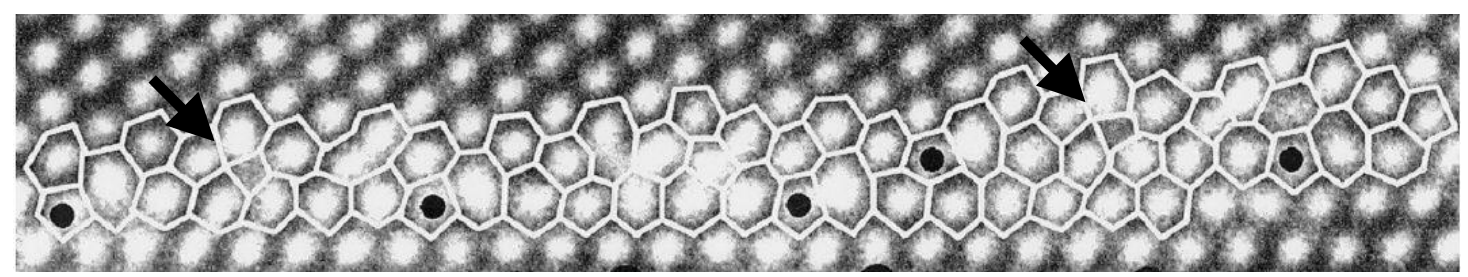

Fig.5: Occurrence of 4 atom ring configurations (arrows) for the $1 / 3<11 \overline{2} 0>$ edge dislocation observed in a non symmetric $\Sigma=7$ grain boundary (Black dots delimit the unit periods of $\Sigma=7$, and the white lines the different atom rings: 6 for the perfect crystal, 5/7, 8 and 4 for the cores of the $1 / 3<1 \overline{2} 0>$ edge dislocation) [18].

Simultaneously, a report was issued using High Angle Annular Dark Field (HAADF) (Zcontrast) imaging showing that the edge dislocation atomic structure was made of 8 atom core [31]. It was argued that such a core should be the only one occurring in GaN in agreement with the theoretical predictions that tried to explain the good electrical and optical properties of GaN layers. For such low electrical activity, the three-fold coordinated atoms in the core had to form dimers along the $<0001>$ line direction.

In epitaxial $\mathrm{GaN}$ layers grown at $800^{\circ} \mathrm{C}$ on a $\mathrm{GaN}$ or $\mathrm{AlN}$ buffer layer deposited at $550^{\circ} \mathrm{C}$, low-angle and high angle grain boundaries were found and analysed (Fig. 6) [32]. By electron diffraction, grain boundaries were identified with rotation angles in the range $1.5-22^{\circ}$. The dislocations were still $1 / 3<1 \overline{12} 0>$ edge type as deduced from the use of the invisibility criterion $\vec{g} \cdot \vec{b}=0$ ( $\vec{g}:$ diffraction vector, $\vec{b}:$ Burgers vector).

Three high angle grain boundaries crossing all the GaN layers were perfectly analysed from HRTEM images by combining topological theory [33] and image simulation [30]. They were described by rotations around the $<0001>$ direction and the values of the rotation angle, as determined from the diffraction patterns, were $13.2 \pm 0.2^{\circ}, 19 \pm 0.2^{\circ}$, and $22.0 \pm 0.2^{\circ}$ [18]. It is well known that the atomic structure of low angle grain boundaries is periodic and based on a 
regular array of dislocations. This concept was then extended to specific high angle grain boundaries, establishing that the atomic structure of coincidence grain boundaries is also periodic [34].

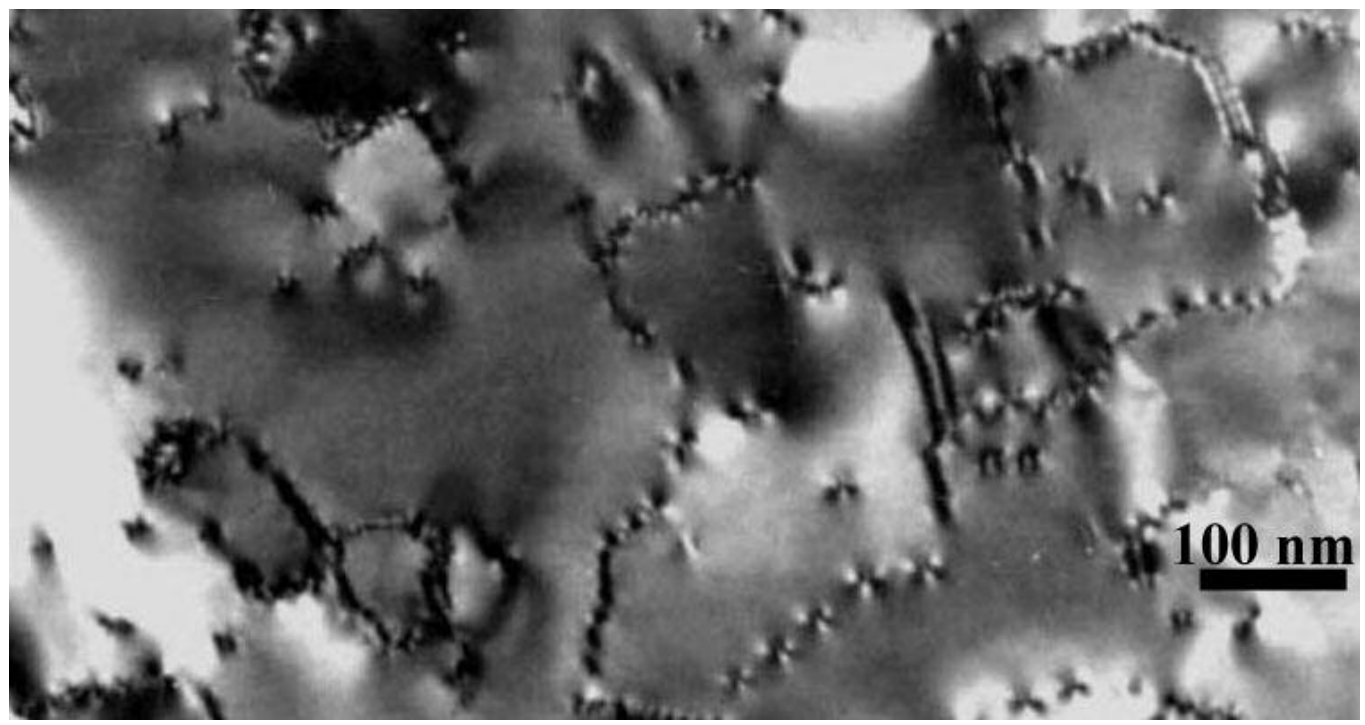

Fig.6: Plan view along [0001] direction showing arrays of dislocations forming low and high angle grain boundaries [32].

These coincidence grain boundaries are defined for specific values of the axis and of the angle of rotation giving rise to a 3D superlattice, the Coincidence Site Lattice (CSL), common to the two adjacent crystals. In the hexagonal system, for rotations around $<0001>$, angles of $13.17^{\circ}, 17.90^{\circ}$ and $21.79^{\circ}$ lead to CSLs which are characterized by their periodic unit cells [35]. The common characteristic of the atomic structures of these coincidence grain boundaries is the regular array of the cores of the edge dislocation $1 / 3<1 \overline{2} 0>$. In Fig.5, between two black dots delimiting the CSL unit cell, 6 atom rings corresponding to the perfect crystal are combined with other atom rings. According to the contrast observed in the HRTEM image and by comparison with the simulated images, the cores were identified as 4 , $5 / 7$ or 8 atom rings. These investigations have allowed us to show that the above dislocation cores are the only structural units, which in addition to the 6 atom unit of the hexagonal 
lattice, can be present in all the possible grains boundaries that form by a rotation around the [0001] direction in $\mathrm{GaN}[18,35]$.

Other extended defects have been shown to exhibit a dislocation character with larger Burgers vectors than unitary lattice parameters. In figure 7 , the circuit around the defect exhibits a closure failure, corresponding to a basal component equal to $2 \vec{a}$ [36]. These defects were identified as nanopipes in comparison to the micropipes observed in $\mathrm{SiC}$ [37], but with rather small radii in contrast to the previsions of the Frank formula [38].

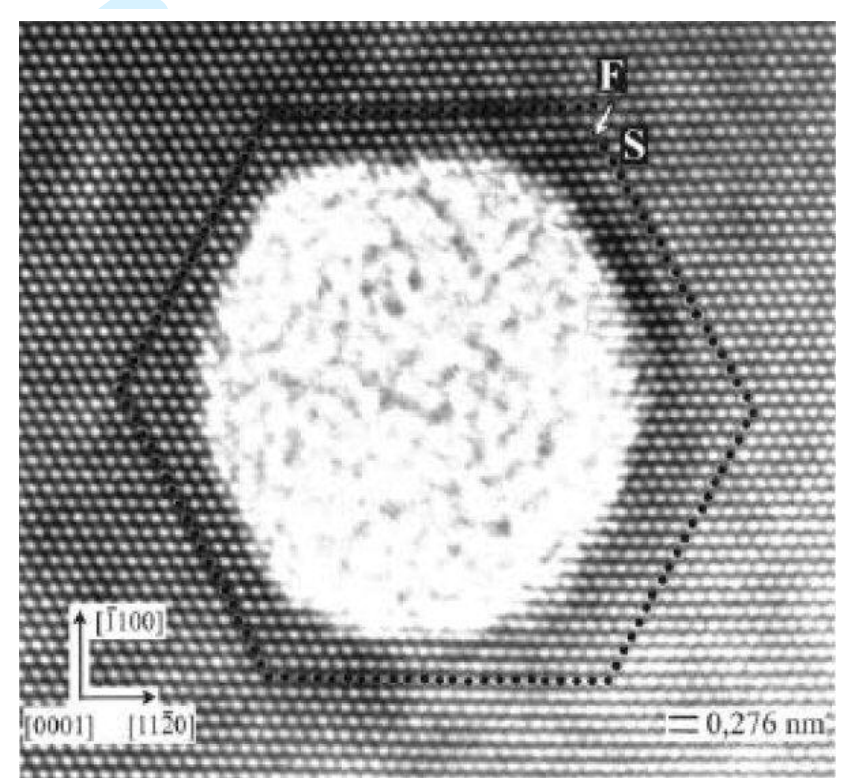

Fig.7: A nanopipe with a Burgers edge component of $2 \vec{a}[36]$

In conclusion, the atomic structures of the cores of the $1 / 3<1 \overline{12} 0>$ prismatic edge dislocations in GaN analysed by HRTEM combined with simulated images present a multiple atomic configuration: three atomic structures were identified ( 8 atom ring or full core, 5/7 atom ring or open core and 4 atom ring). The 8 atom ring or full core was the only one identified by HAADF. 


\subsubsection{Screw prismatic dislocations}

The first investigations of the surface of GaN layers, grown by MOCVD at $1040^{\circ} \mathrm{C}$ on an AlN buffer layer deposited at $450-500^{\circ} \mathrm{C}$, using scanning force microscopy have shown the presence of tunnel-like defects aligned along the $<0001>$ growth direction. The radii of theses tunnels are in the $3.5-50 \mathrm{~nm}$ range and they propagate along the growth direction. These defects are called nanopipes. These experimental investigations have also shown that the screw dislocation associated with a nanopipe emerges on the top surface as a wide crater with 60-100 nm radii. By combining these observations with HRTEM analysis, it was concluded that the nanopipes are the open cores of screw dislocations $(\vec{b}=0001)$ [39]. This result was later confirmed by showing that such defects may alternate from an open (nanopipe) to closed core along their way to the surface of the epitaxial layer (classical character of a dislocation) [40]. The atomic structure of full core screw dislocations was reported in a high resolution Zcontrast analysis [31]. The growth mode can affect the dislocation core and as was shown in hydride vapour phase epitaxy (HVPE) grown materials, screw dislocations are decorated by voids or pinholes [41]. The fluctuations in the intensity of the atomic columns in HRTEM of the HVPE specimens have been attributed to local excess of gallium atoms in the core [42].

\subsection{Basal dislocations}

GaN layers deposited on top of the (0001) sapphire are characterised by the well known epitaxial relationship: $(0001)_{\mathrm{sap}} / /(0001)_{\mathrm{GaN}}$ and $[1 \overline{12} 0]_{\mathrm{sap}} / /[10 \overline{1} 0]_{\mathrm{GaN}}[43]$. The network of anions is continued across the interface. The misfit is large $(-16.07 \%)$ and it is accommodated by misfit dislocations in the basal plane. The possible Burgers vectors of the perfect dislocations are $1 / 3<1 \overline{120}>$, and the line directions are $<1 \overline{120}>$ leading to pure screw or $60^{\circ}$ dislocations according to the angle between the Burgers vector and the line direction. 
After Bragg filtering of HRTEM images, it was shown that this lattice misfit is relaxed in the first monolayer of $\mathrm{GaN}$ with a network of regularly spaced $60^{\circ}$ dislocations. A residual stress of $-2.1 \%$ was evaluated near the interface and related to the high density of threading dislocations present in the epitaxial layer [44].

Since threading and misfit dislocations are always present, investigations were carried out at an interface between AlN and sapphire in order to analyse the connection of these two dislocation networks [45]. Molecular Beam Epitaxy (MBE) was used for the deposition of the AlN buffer on $\mathrm{Al}_{2} \mathrm{O}_{3}$, at $800^{\circ} \mathrm{C}$, until a thickness of $40 \mathrm{~nm}$ was reached. In HRTEM plan view images, a set of three-fold moire patterns due to the overlap of the $\{10 \overline{10}\}_{\text {AIN }}$ and $\{1 \overline{2} 0\}_{\text {sap }}$ planes is formed at the layer-substrate interface with a mean spacing of $2.16 \mathrm{~nm}$ instead of $2.029 \mathrm{~nm}$ for the theoretical value. This discrepancy means that $7 \%$ of the compressive stress is not relaxed by the misfit dislocations. From the moiré pattern, the occurrence of threading dislocations is readily deduced from the terminating moiré fringes (Fig.8).

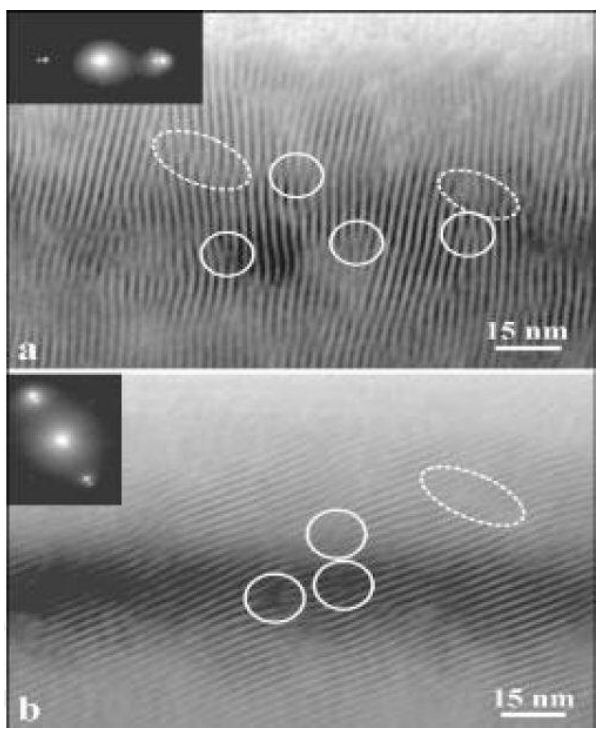

Fig.8: Plan-view HRTEM image showing a translation moiré pattern (a) one set of reflections, (b) a second equivalent set [45]

The dislocation density measurements from various areas of the moiré pattern showed an average density in the order of $1.5 \times 10^{11} \mathrm{~cm}^{-2}$ with a lower rate near the top surface of $8 \times 10^{10}$ $\mathrm{cm}^{-2}$. In figure 9 , the white arrows indicate the terminating fringes due to the threading 

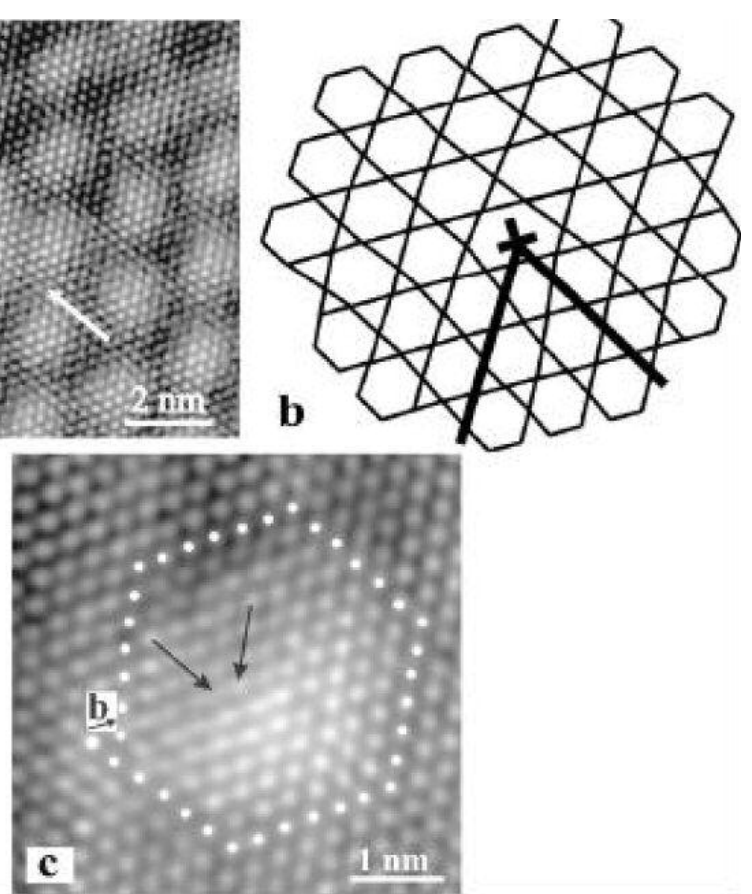

dislocation marked by the black fringes and corresponding to two extra prismatic half planes. The circuit around the defect gives as Burgers vector $\vec{a}$ suggesting an edge or mixed type dislocation.

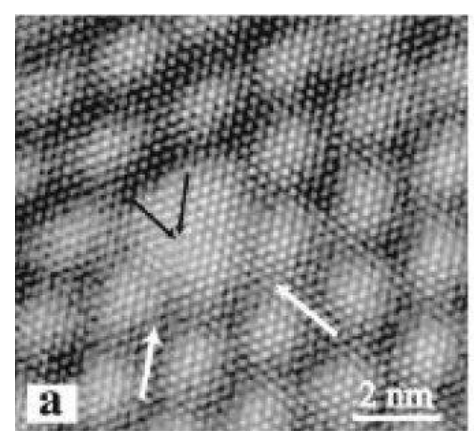

Fig.9: (a) Threading dislocations imaged by the terminating moiré fringes (b) Schematic illustration of the misfit network

(c) Fourier filtered image showing only the AlN structure [45]

The corresponding missing half-planes are associated with two misfit dislocations of the interfacial network as shown in Fig.9b. The Burgers vector of each threading dislocation is equal to the algebraic sum of the Burgers vectors of the two associated misfit dislocations. Similar observations with moiré analysis were carried out on HVPE grown layers on SiC substrates [46]. Therefore, threading dislocations existing in $\mathrm{GaN}$ films can be related to the misfit dislocations at the interface with the substrate. This view is complemented by other investigations which show that many threading dislocations can originate inside the buffer layer due to the recombination of stacking faults and subsequent climb of the dislocations in the prismatic planes [47]. 


\subsection{Partial dislocations}

In MOCVD layers grown on (0001) sapphire, evidence of dissociation in the basal plane was shown using the weak beam technique and the following mechanism was proposed: $\vec{c}+\vec{a}=\left(\vec{c} / 2+\vec{b}_{1}\right)+\left(\vec{c} / 2+\vec{b}_{2}\right)$, with $\vec{b}_{1}$ and $\vec{b}_{2}$ being Shockley partials, corresponding to the formation of an intrinsic stacking fault $\mathrm{I}_{1}$ (Ponce et al., 1996). However, after introduction of dislocations by indentation, dissociation of the perfect screw dislocation or $60^{\circ}$ dislocation into partial dislocations was not observed in the limit of the weak beam resolution, $2 \mathrm{~nm}$, however stacking faults were present in the nodes formed by the screw dislocations [48]. In hexagonal structures, three basal faults are possible: two intrinsic $\mathrm{I}_{1}$ and $\mathrm{I}_{2}$ and one extrinsic $\mathrm{E}$. $\mathrm{I}_{1}$ is formed by removing the B plane above the A plane and shearing the remaining part by $1 / 3<10 \overline{1} 0>, I_{2}$ is formed by considering only the shear, and E by inserting one C plane between two adjacent basal planes. The energy of the basal stacking faults was calculated using DFT: $\mathrm{I}_{1}=18, \mathrm{I}_{2}=43$ and $\mathrm{E}=69 \mathrm{~mJ} / \mathrm{m}^{2}[49,50]$. They are of the low-energy type. $\mathrm{In}$ HRTEM, the density of stacking faults near the interface with the substrate is very high, most of them are of the $I_{1}$ and $I_{2}$ types even if segments of the $E$ fault were also observed and partial dislocations were identified.

The $\mathrm{I}_{1}$ fault was interpreted as the climb-dissociation process:

of the $1 / 3<1 \overline{120}>$ following the reaction:

$$
1 / 3<1 \overline{2} 0>1 / 6<20 \overline{2} 3>+1 / 6<02 \overline{2} \overline{3}>
$$

or of the $<0001>$ perfect dislocation:

$$
<0001>\quad \rightarrow 1 / 6<20 \overline{2} 3>+1 / 6<2 \overline{2} 03>
$$

The $I_{2}$ fault results from the shear of the structure leading to a partial dislocation loop, the two partial dislocations bounding the basal fault have opposite Burgers vectors $1 / 3<10 \overline{1} 0>$ [51]. Partial dislocations, $1 / 3<10 \overline{10}>$, can also be formed at the intersection of a basal stacking 


\subsubsection{Scanning probe microscopy investigations}

In their leakage current comparative work on $\mathrm{GaN}$ based devices from layers grown by HVPE and MBE, Hsu et al. showed that the reverse bias leakage was not uniformly distributed in the sample [53]. A small portion of the sample was found to be responsible for the majority of the leakage. The correlation between reverse bias leakage locations and topographic hillocks in samples suggested that reverse bias leakage primarily occurred at screw/mixed dislocations. Further confirmation was given from the similar density between the leakage spots and screw/mixed dislocation density determined by TEM. The fact that samples had distinctly different morphologies but showed similar density of leakage spots led to the conclusion that the origin of the reverse bias leakage was the same in the set of samples. They showed that screw/mixed dislocations contribute significantly more to gate leakage than 
pure edge dislocations. This was in agreement with other reports where screw/mixed dislocations had been found to be more effective recombination centers [54], and to have reduced barrier heights compared to pure edge dislocations [55]. In such devices, the reverse bias leakage can be orders of magnitude different for samples grown under slightly different conditions. For example, the reverse bias current was orders of magnitude higher for Ga-rich samples. Given that dislocations with a screw component are responsible for the reverse bias leakage and their density is the same for the HVPE template and the two MBE/HVPE films, these results led to conclude that the dislocation electrical activity was sensitive to local chemical and/or structural changes, which depend on growth methods and conditions.

\section{4. 2. Cathodoluminescence investigations}

Correspondence between the individual dislocations and the active centres in MOVPE GaN layers by combining TEM and cathodoluminescence has shown that the dislocations are non-radiative recombination centers [56]. Remmele et al. [57] were able to distinguish the $\vec{a}$ threading dislocations and $\vec{a}+\vec{c}$ dislocations in their bright field images, since the $\vec{c}$ component of the $\vec{a}+\vec{c}$ dislocations caused a black and white lobe contrast due to surface relaxation effects [58]. The $\vec{a}$ dislocations showed only a dark contrast. The comparison of the bright field images and the monochromatic CL mapping (band edge luminescence) gave the following results: (i) the major part of the $\vec{a}+\vec{c}$ dislocations is not recombination active. (ii) $\vec{a}$ threading dislocations reduced the band edge luminescence, thus representing centres of non-radiative recombination, (iii) $\vec{a}$ dislocations in the basal plane caused non-radiative recombination.

\section{Atomistic simulation of dislocations in GaN}

The main goal of atomistic modelling of dislocations is to find realistic models of the cores and provide information on the microscopic mechanisms occurring in this area. From such studies one expects to derive, at least, two types of information. The first is to identify 


\subsubsection{The ab initio methods}

In the ab initio methods, the interatomic interactions are described rigorously in the framework of quantum mechanics. Among the ab-initio methods, those based on DFT are largely used in the field of computational materials science $[59,60]$. In these latter methods, the electronic density plays a key role; the quantities of interest in atomistic simulation: energy and forces are derived from it. The electronic density is obtained by solving the selfconsistent Kohn-Sham equations [60]. To perform ab initio DFT calculations in a tractable way, a number of approximations are necessary. The most important are made on the form of the exchange and correlation functional (Local Density Approximation, Generalised Gradient Approximation ...etc.) and on the basis set used to represent the single-electron wave function (plane waves, Gaussian type functions, Slater type functions). Moreover, to make these calculations more tractable, the pseudopotential approximation is usually used [61]. Despite these approximations, the ab initio based DFT calculations remain computationally expensive and their applications are limited to systems of a few hundred atoms (typically 200 atoms). 
Ab-initio-DFT methods using pseudopotentials were implemented in several codes like: VASP (Vienna Ab-initio Simulation Package) [62], FHI-md (Fritz-Haber Institute's Molecular Dynamics code) [63], AIMPRO (Ab Initio Modelling PROgram) [64] and SIESTA (Spanish Initiative for Electronic Simulations with Thousands of Atoms) [65]. While the first two use a representation based on the plane waves, AIMPRO and SIESTA use Gaussian-type and numerical Slater-type atomic orbital, respectively. Except SIESTA, these codes have been used in different investigations of dislocations in GaN: VASP [15, 16, 66], FHI-md [23] and AIMPRO $[2,22,67,68]$.

\subsubsection{The empirical potentials}

Less sophisticated than ab initio and tight-binding methods, the empirical potentials allow dealing with systems of many thousands of atoms, and this of interest for dislocation modelling [69]. In empirical potential methods, one postulates a relatively simple analytical form of the potential energy including a set of adjustable parameters. These are fitted to experimental measurements or ab initio calculations in order to reproduce a set of properties of the material under investigation (lattice parameter, elastic constants, bulk modulus, phonon spectrum,). It is assumed that the approximated form of the empirical potential will reproduce the main feature of the atom-atom interactions and that the number of adjustable parameters is sufficient to ensure its transferability.

Some empirical potential have previously been used to calculate the energies of dislocations and grain boundaries in semiconductors [70-73]. They brought about a good insight on the intrinsic properties of elemental semiconductors. Keating and Baraff potentials are limited by their parameterisations which exclude dangling bonds. However, the two other potentials: Stillinger-Weber and Tersoff, take into account different atomic surroundings. The Stillinger-Weber potential has been used for III-V compound semiconductors [20, 74-76]. Of 


\subsubsection{The tight-binding methods}

Coming right in between the $\mathrm{ab}$ initio and empirical potential methods, tight-binding methods combine a minimal amount of quantum mechanics of electrons with classical potentials. This allows achieving more transferable description of the interatomic interactions than empirical potentials, at a small fraction of the computational cost of ab-initio calculations. Consequently, tight-binding methods are appropriate to model systems with many hundred to a thousand atoms and can be applied to deal with problems beyond the reach of ab-initio methods. In standard tight-binding methods, the total energy of the system is approximated by a sum of two terms [79]. The first, called the band structure energy, contains the quantum electronic effects; it is obtained by solving non self-consistent Kohn-Sham likeequation [80]. Here, the single electron wave functions are represented by linear combinations of atomic orbital. Hamiltonian and overlap matrix elements are evaluated in the framework of the two-centre approach of Slater-Koster [81]. The second is a short-ranged diatomic potential, which is fitted to ab-initio calculations or experimental measurements in order to reproduce a selected set of materials properties. Better accuracy and transferability is obtained using the self-consistent tight-binding methods [82]. In these methods, an additional term is added to the energy functional and adjusted through a self-consistent procedure. This is useful 
to take into account the charge transfer occurring in compound semiconductors like GaN. The Self-Consistent Charge Density Functional Tight-Binding (SCC-DFTB) [83-85] is a selfconsistent extension of the standard DFTB method [86]. The SCC-DFTB total energy was derived by expanding the Kohn-Sham energy functional to the second order with respect to the electronic density fluctuations around a given reference density [80, 83]. Besides the two usual energetic terms, i.e. the band structure energy and the short-ranger diatomic potential, a third term is introduced where a self-consistent procedure is incorporated at the level of Mulliken charges to account for charge transfer. This last term allowed to the SCC-DFTB technique to achieve accuracy comparable to that of ab-initio methods. Among the tightbinding methods, the SCC-DFTB method is one of most successful as applied to dislocations in $\mathrm{GaN}[2,78,87-91]$.

\subsection{Results of dislocation modelling in GaN}

From 1997, there has been a tremendous effort in the field of atomistic modelling to study dislocations in GaN. These studies attempt to provide some information about the core configuration of these dislocations and their effect on the electronic performance of the grown layers. Several kinds of dislocations have been investigated in wurtzite GaN; these were perfect or partial with edge, screw or mixed character, belonging to the prismatic or the basal plane.

\subsubsection{Perfect prismatic edge dislocation}

The prismatic edge dislocation $(\vec{b}=a / 3<11 \overline{2} 0>, l=<0001>)$ was the first dislocation to be investigated in wurtzite GaN [2]. This dislocation displays different core structures with stoichiometric configurations (8,5/7 and 4 atom rings) (Fig.10) or non-stoichiometric configurations $\left(8-\mathrm{V}_{\mathrm{Ga}}\right.$ and $8-\mathrm{V}_{\mathrm{N}}$ atom rings). The core with 8 atom ring structure or full-core contains a column of three fold coordinated atoms whereas the 5/7 atom ring structure or 
Page 63 of 83

Philosophical Magazine \& Philosophical Magazine Letters

open-core contains wrong bonds (Ga-Ga, N-N). The core with 4 atom ring structure contains fully coordinated atoms establishing four in-plane bonds [20]. The non-stoichiometric $8-\mathrm{V}_{\mathrm{Ga}}$ or $8-\mathrm{V}_{\mathrm{N}}$ cores can be obtained from the core with 8 atom rings removing some of $\mathrm{Ga}$ or $\mathrm{N}$ atoms from the central column, while the complete removal of this column leads to the $5 / 7$ core $[2,14,21,66,88,92]$. Béré et al. have shown that the different cores $(8,5 / 7,4)$ are, in fact, obtained in a straightforward manner only by changing the origin of the displacement elastic field during the generation of the model [21]. In the framework of ab-initio and tightbinding (SCC-DFTB) calculations, Elsner et al. [2] reported that the core with 8 atom ring structure is more energetically favorable than that with $5 / 7$ atom ring structure. However, all the subsequent calculations performed with different methods: empirical potentials -Modified Stillinger-Weber- [20, 76], tight-binding -SCC-DFTB- [88] and ab initio [23] suggest the opposite. In the case of the core with 4 atom ring structure, Chen et al. found it less energetically favorable than that with 8 atom ring structure [20].

(a)

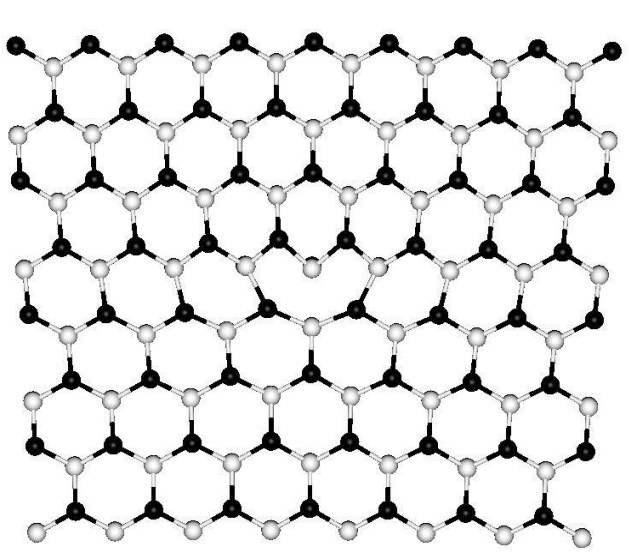

(b)

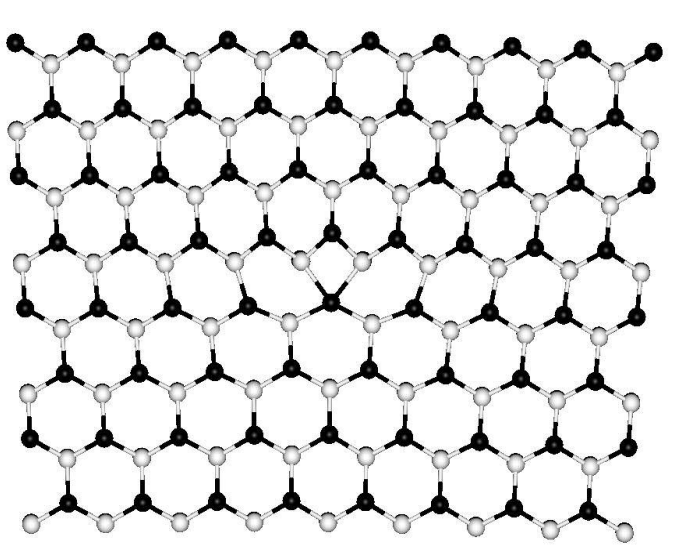

(c)

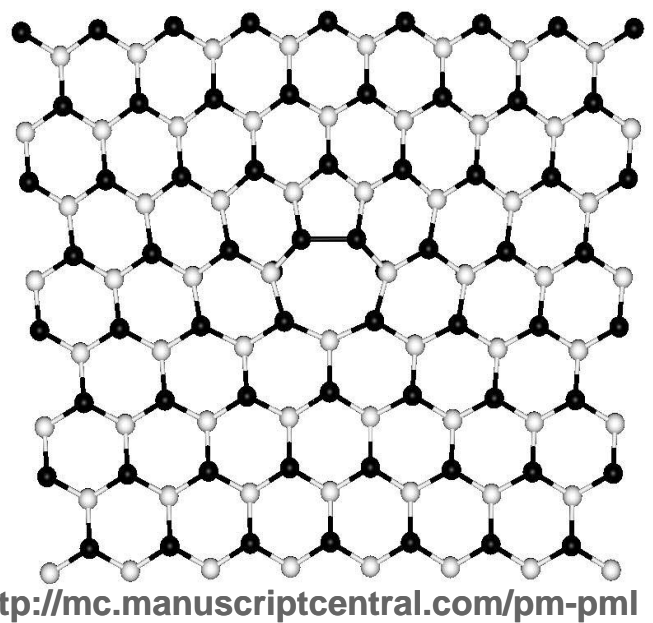

23 
Fig. 10: View along the [0001] direction of the atomic core configurations of the prismatic edge dislocation. a) 8 atom ring core, b) 4 atom ring core, c) 5/7 atoms ring core.

More recently, the core with 4 atom ring structure was reinvestigated by means of multiscale calculations: ab-initio, empirical potential and continuum elastic theory [23]. The authors showed that under a tensile strain, the 4 atom ring core becomes the most energetically favorable among the three cores of the stoichiometric edge threading dislocations.

Since the structure of tilt grain boundaries is described with a periodic array of the edge dislocation, they were investigated using our empirical potential. They were generated through rotations around the [0001] direction and it was then possible to show that the concept of structural units was valid for the hexagonal system [35]. In this instance, the building units for all grain boundaries were the 6 atom ring for the perfect lattice and the 5/7 atom core for the grain boundary dislocations (Fig. 11) [93].
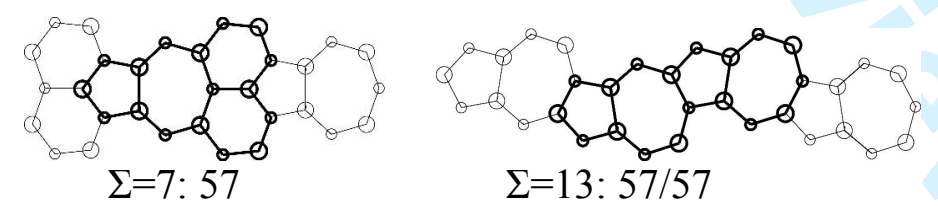
$\Sigma=13: 57 / 57$

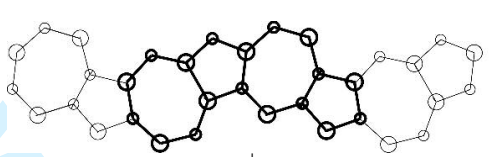

$\Sigma=13: 57^{+} 57^{-}$

Fig.11: Structural units for coincidence grain boundaries from rotations around [0001] based on the 5/7 atom ring core, the corresponding $\Sigma$ is indicated [93]

This was a good indication that the hexagonal system did not exhibit fundamentally different mechanisms for defect formation than the cubic, at least along high symmetry directions.

A set of calculations based respectively on ab-initio [66], tight-binding -SCC-DFTB[88] and Monte-Carlo [92] methods revealed that the formation energy of the prismatic edge dislocations with non-stoechiometric cores $\left(8-\mathrm{V}_{\mathrm{Ga}}\right.$ and $\left.8-\mathrm{V}_{\mathrm{N}}\right)$ strongly depends on both the growth conditions and the doping nature of the material. The $8-\mathrm{V}_{\mathrm{Ga}}$ core was shown to be 
more energetically favourable in n-type material grown under N-rich conditions whereas the $8-\mathrm{V}_{\mathrm{N}}$ core is favourable in p-type material grown under Ga-rich conditions [66]. However, Lee et al. obtained favorable formation energies for cores with both 8 and $5 / 7$ atom rings structures in material grown under Ga-rich conditions [88].

The electronic structure of the prismatic perfect edge dislocation with different core configurations has also been investigated extensively [2, 23, 66, 67, 88]. According Elsner et al. the edge dislocation with 8 atom ring core structure is electrically inert and induces no gap states [2]. Therefore, it has undergone a relaxation of the three fold coordinated $\mathrm{Ga}(\mathrm{N})$ core atoms toward $\mathrm{sp}^{2}\left(\mathrm{p}^{3}\right)$ similar to the $(10 \overline{1} 0)$ surface, which leads the dimerization of the bonds along the dislocation line. However, several calculations performed on the same core configuration have revealed the presence of empty states at the half top of the band gap [22, $23,67,88]$. A quite similar electronic structure has been reported for the dislocation with $5 / 7$ atom ring core structure (Fig.12) [23, 88].
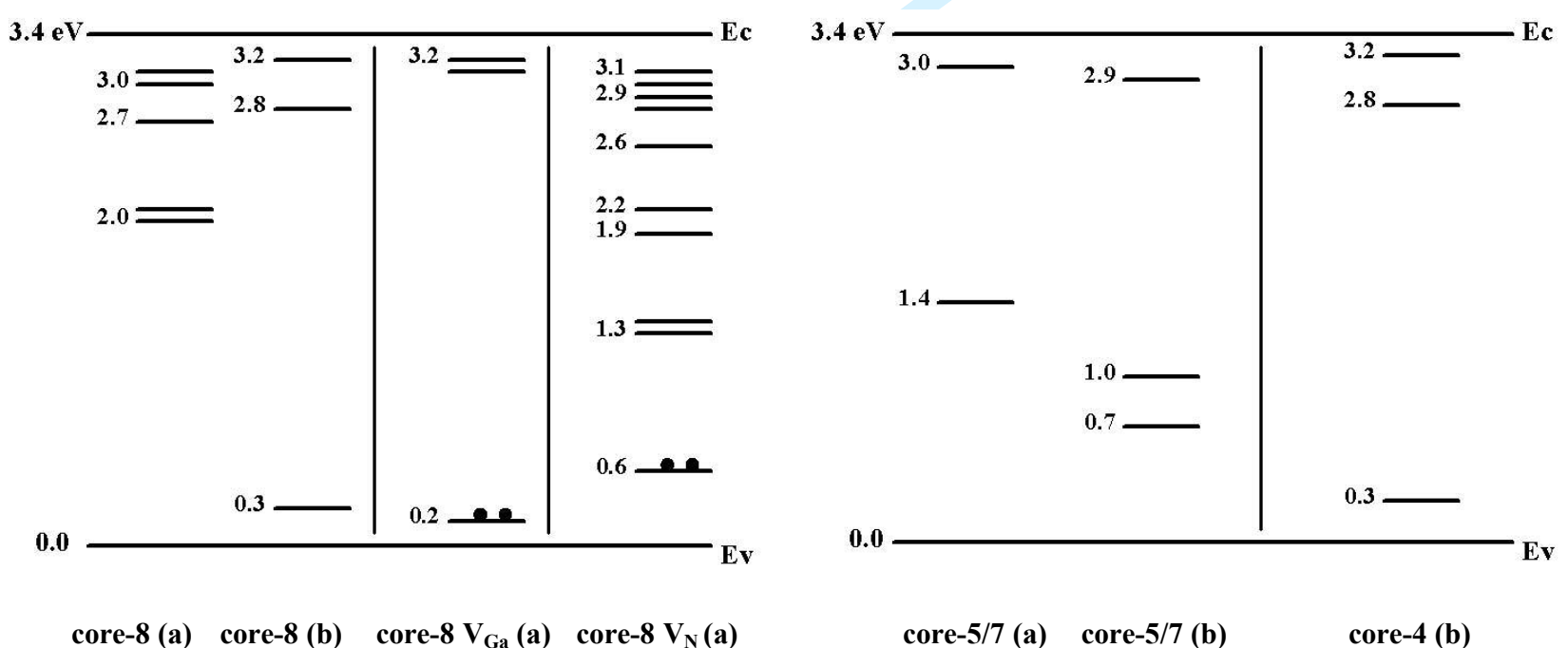

Fig.12: Schematic diagram of energy levels induced in the band gap by different cores of the prismatic edge dislocation: 8 atom ring core (core-8), 5/7 atom ring core (core-5/7) and the 4 atom ring core (core-4), dark balls indicate filled levels, (a) [88], (b) [23] 
For the 4 atom ring edge dislocation, an unexpected behavior has been reported recently: despite its fully coordinated core, it exhibits deep states in the band gap [23]. This was attributed to the large strain field surrounding the dislocation core which leads to the formation of bonding between Ga second neighbor atoms similar to metallic gallium. TightBinding calculations carried out on the non-stoichiometric structures have revealed for the 8$\mathrm{V}_{\mathrm{Ga}}$ core, the presence of both occupied states at $0.2 \mathrm{eV}$ from the VBM (Valence Band Maximum) and empty states just below the CBM (Conduction Band Minimum) [88]. The 8$\mathrm{V}_{\mathrm{N}}$ core has been shown to induce different states throughout the band gap; it may be expected to be an origin of yellow luminescence.

The electronic structures of the $(3 \overline{7} 40) \Sigma=37\left(\theta=9.3^{\circ}\right)$, $(3 \overline{5} 20) \Sigma=19\left(\theta=13.4^{\circ}\right)$ and $(1 \overline{3} 20) \Sigma=7\left(\theta=21.6^{\circ}\right)$ tilt grain boundaries were calculated. They were investigated by means of SCC-DFTB approach. Among the three possible atomic configurations of these boundaries, namely, the interfaces based on the $5 / 7$, the 4 and the, it was found that the structure based on the 8 atom ring core introduces deep states in the very center and the upper half of the band gap whereas the grain boundaries based on the $5 / 7$ atom ring possess only states close to the conduction band (Fig.13) [90].

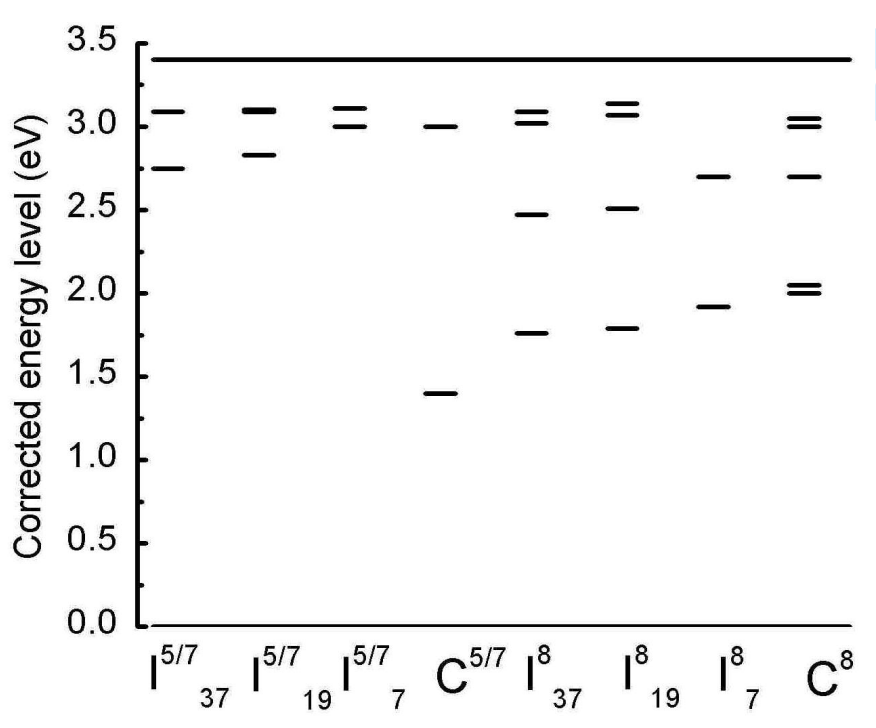




\subsubsection{Perfect prismatic screw dislocation}

The prismatic perfect screw dislocation $(\vec{b}=c<0001>, l=<0001>$,) is one of the most studied dislocation in wurtzite GaN. Several calculations have been carried out on their atomic and electronic core structures. Different core structures have been investigated: the full-core $[2,67,76,89]$, the open-core $[2,15]$ and non-stoichiometric configurations $[15,16]$ (Fig.14). In the first configuration, six stoichiometric atomic columns, in helical like symmetry arrangement along the direction [0001], form a hexagonal atomic core. By removing the latter, the open-core configuration is obtained. However, filling the core with different amounts of $\mathrm{Ga}$ and $\mathrm{N}$ atoms leads to non- stoichiometric configurations, where the Ga-filled and N-filled cores represent the extreme limits. In their report, Elsner et al. have investigated prismatic screw dislocation with both full-core and open-core configurations [2]. The calculation of the line energy, in a cylinder of radius about $8 \AA$, gave $4.88 \mathrm{eV} / \AA$ for the full-core and $4.55 \mathrm{eV} / \AA$ for the open-core configuration. This was higher than their value of $2.19 \mathrm{eV} / \AA$ found for the full-core edge prismatic dislocation. Similar high line energy values 
were also reported by different authors for the screw full-core dislocation. This probably explains why the screw dislocations represent a small fraction with respect to the edge type in GaN epitaxial layers [15, 76, 89].

Recent ab initio calculations performed by Northrup $[15,16]$ revealed that screw dislocation with non-stoichiometric core configurations could be more energetically favourable than those with stoichiometric cores under some growth conditions. The Ga-filled core, obtained by removing all the $\mathrm{N}$ atoms from the hexagonal core, is predicted to be the most stable configuration under the Ga-rich conditions [15]. However, in N- rich conditions, a non-stoechiometric core structure with $\mathrm{Ga}(50 \%)$ and $\mathrm{N}(25 \%)$ atoms was found more energetically favourable [16].

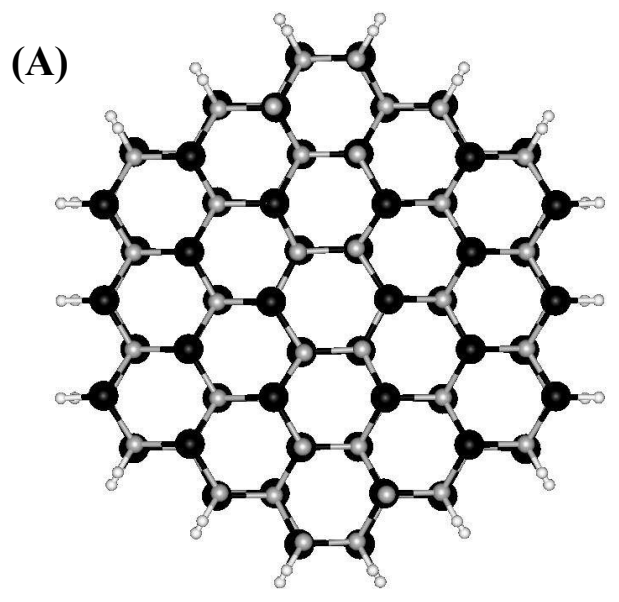

(B)

(C)
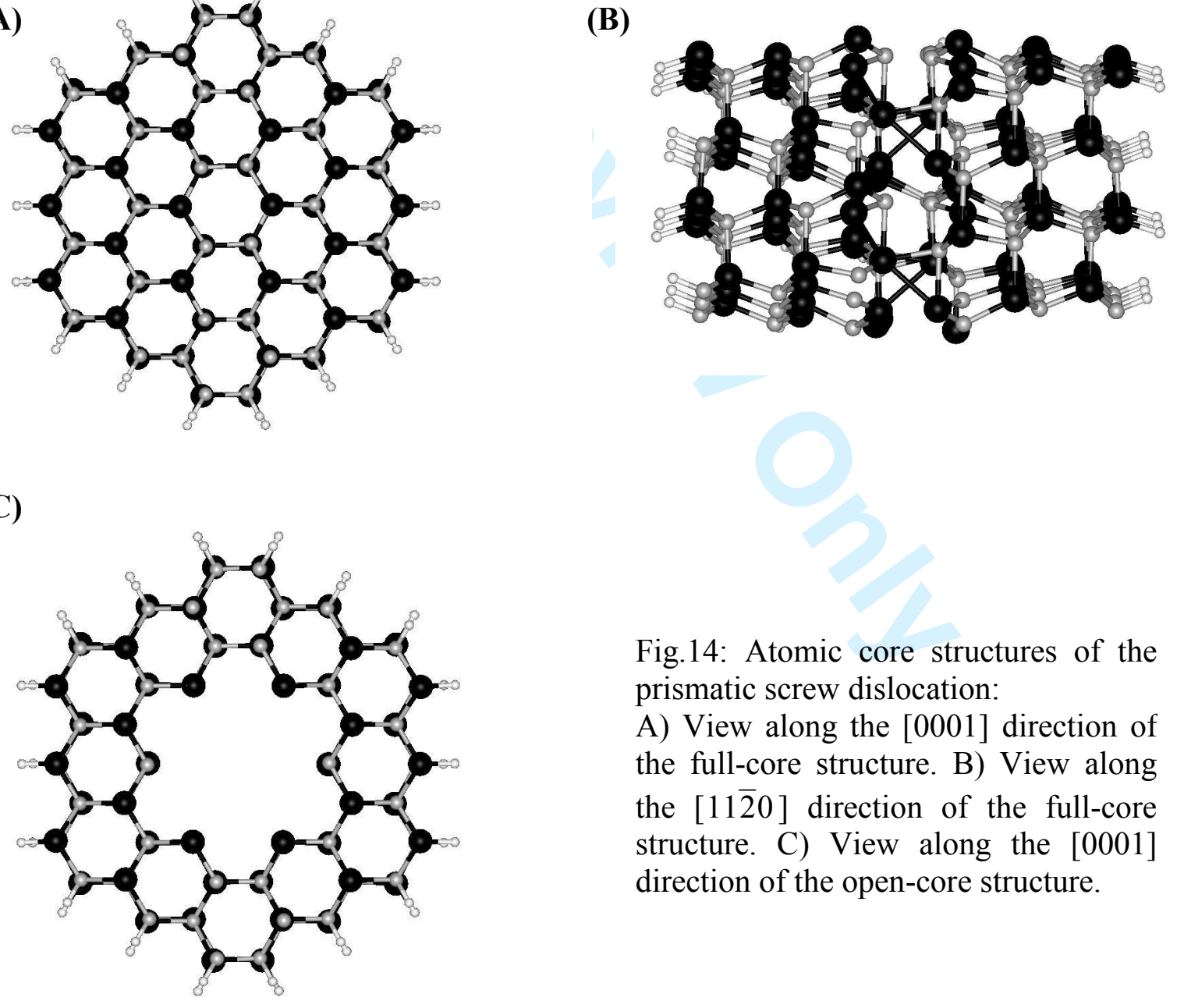

Fig.14: Atomic core structures of the prismatic screw dislocation:

A) View along the [0001] direction of the full-core structure. B) View along the $[11 \overline{2} 0]$ direction of the full-core structure. C) View along the [0001] direction of the open-core structure. 
In the full-core configuration, due to the presence of heavily distorted bonds, additional charge transfers should occur and gap states are induced. Belabbas et al. have identified an extra charge transfer of $0.12 \mathrm{e}$, besides that naturally occurring in $\mathrm{GaN}$ bulk material, from $\mathrm{Ga}$ to $\mathrm{N}$ atoms [89]. The electronic structure calculations performed on the full-core dislocation have revealed the presence in the band gap of shallow as well as deep states $[2,67]$. In the open-core dislocation, in order to lower the surface energy, the internal wall of the core relaxes like the $(10 \overline{1} 0)$ surface and then the threefold coordinated atoms $\mathrm{Ga}(\mathrm{N})$ adopt sp2 $\left(\mathrm{p}^{3}\right)$ hybridization. For this dislocation, only shallow states were reported and attributed to the distortion of the wall surface along the direction [0001] due to the Burgers vector [2]. For the non-stoichiometric dislocations, the Ga-filled core configuration was predicted to have a metallic like-behaviour as its induced states are highly dispersed in the entire band gap [15, $16,67]$. However, this behaviour is less pronounced in the most favourable core structure (Ga $50 \%, \mathrm{~N} 25 \%$ ) in N-rich conditions [16].

\subsubsection{Perfect basal edge and screw dislocations}

Up to date, there are only a few reports on the atomic core structures of the perfect basal edge $(\vec{b}=c<0001>, l=<11 \overline{2} 0>)$ and screw $(\vec{b}=a / 3<11 \overline{2} 0>, l=<11 \overline{2} 0>)$ dislocations [76, 78]. In the ELO (Epitaxial Lateral Overgrowth) process, the threading dislocations are bent [94]. Thus, a dislocation changes its line direction from $<0001>$ to $<11 \overline{2} 0>$ resulting in a new character, edge or screw. These basal dislocations can occur during the heteroepitaxial growth

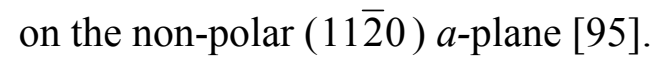

Recently, Belabbas et al. have investigated the atomic structure and the electronic activity of the basal edge dislocation in wurtzite $\mathrm{GaN}$ by combining empirical potentials (Modified Stillinger-Weber) and tight-binding methods (SCC-DFTB)[78]. For this dislocation 
four stable core configurations have been determined, and each corresponds to a particular position of the origin of the applied displacements field before relaxation (Fig.15a) [21]. G1 and G2 configurations belong to the glide set, S1 and S2 to the shuffle set. The G2 and S1 configurations display a 4/8 atom ring structure without wrong bonds (Fig.15c, d), whereas the G1 and S2 configurations which include wrong bonds, display a 5/8/5-atom rings structure (Fig.15b, e). The G1 and S2 configurations were already reported in the framework of empirical potentials [76].

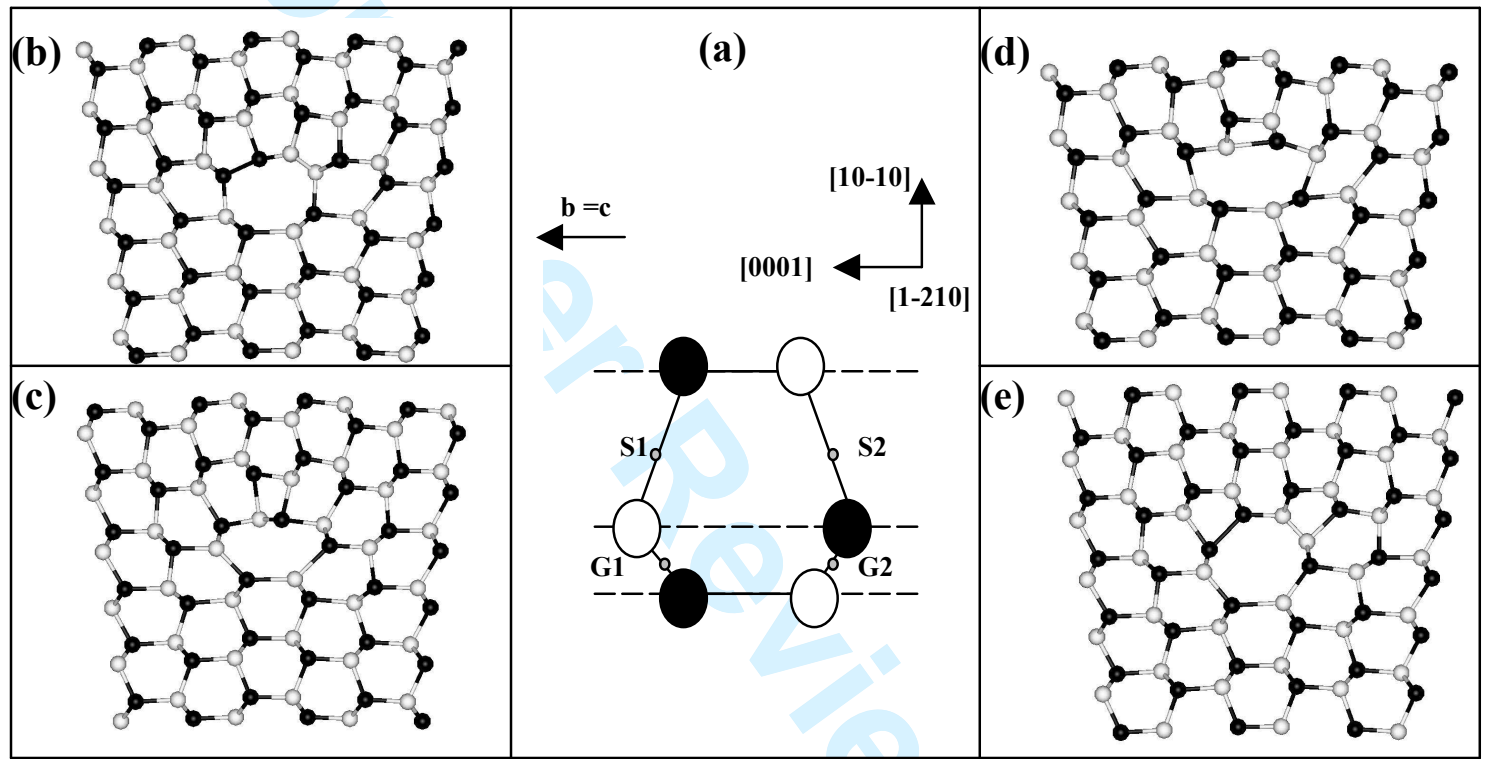

Fig.15: The four core configuration of the $\vec{c}$ edge basal dislocation: (a) Different positions of the origin of the applied displacements field, (b) G1 core configuration, (c) G2 core configuration, (d) S1 core configuration, (e) S2 core configuration. Black circles represent $\mathrm{Ga}$ atoms whereas white ones represent $\mathrm{N}$ atoms.

The SCC-DFTB energetic calculations for a core radius of $8.58 \AA$ gave for the four configurations: G1, G2, S1, S2, the following core energies: 5.24, 4.90, 5.73 and $4.44 \mathrm{eV} / \AA$, respectively [78]. In both sets, the most favourable configuration is S2 with wrong bonds. In the glide set, the core without wrong bonds, G2, is energetically favoured. The electronic levels induced by each core configuration are presented in figure 16, where their positions in the band gap were corrected using the linear scaling method. 


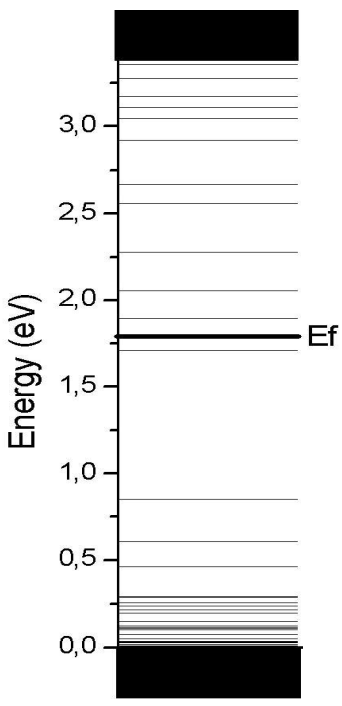

(a) G1 configuration

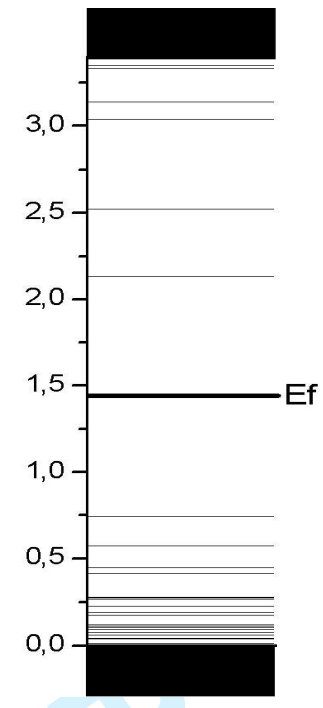

(b) G2 configuration

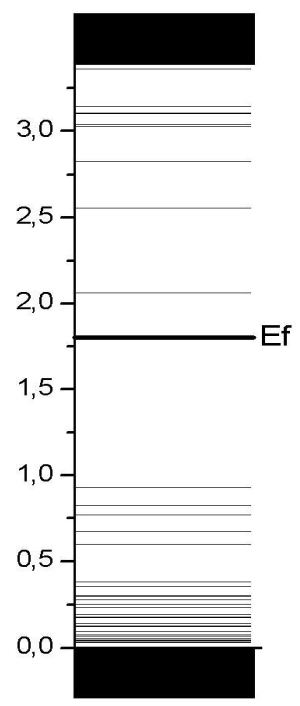

(c) S1 configuration

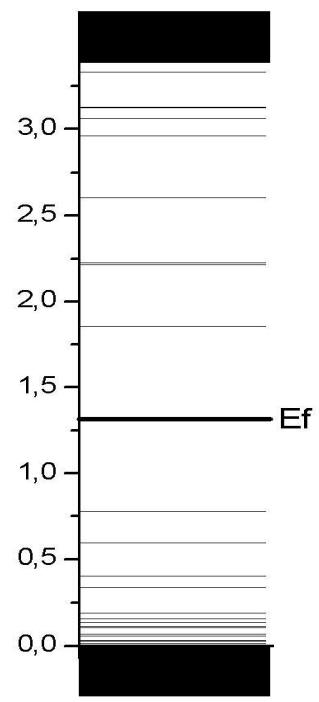

(d) S2 configuration

Fig. 16: Electronic gap states associated with different configurations of the basal edge dislocation. The zero has put on the top of the valence band maximum while the conduction band minimum is at $3.4 \mathrm{eV}$.

As can be seen, each core configuration induces deep as well as tail states. Filled states exist in the bottom half of the band gap, up to $1 \mathrm{eV}$ from the VBM. For all the configurations, unfilled deep states are present on the top half of the band gap, and are located mainly between 2 and $3 \mathrm{eV}$, from the VBM. In this area, the states induced by G1 are more spread than those of the other configurations. Due to the presence of the deep states, one could expect the basal edge dislocations to be at the origin of parasitic luminescence. However, it cannot be excluded that the G1 configuration may be involved in non-radiative recombination processes.

The atomic structure of the perfect basal screw dislocation has been only investigated by Béré et al., their study is based on a modified Stillinger-Weber potential [76]. Two stable core configurations were identified; one belongs to the glide set while the other belongs to the shuffle set. The screw dislocations were found to have a core diameter of $6.7 \AA$. The glide 
configuration has a line energy of $1.20 \mathrm{eV} / \AA, 2 \%$ higher than that of the shuffle configuration.

\subsubsection{Perfect basal $60^{\circ}$ dislocation}

In zinc-blende GaN, Blumenau et al. have studied the atomic and electronic structures and the relative energies of the $60^{\circ}$ basal dislocation $(\vec{b}=\mathrm{a} / 2[1 \overline{1} 0], l=<01 \overline{1}>)$, with both glide and shuffle configurations (Fig.17) [22, 95].

(A)

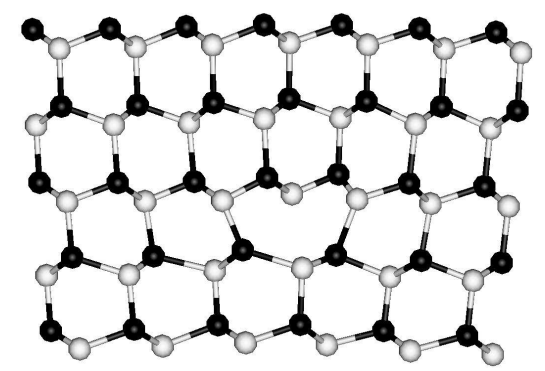

(C)

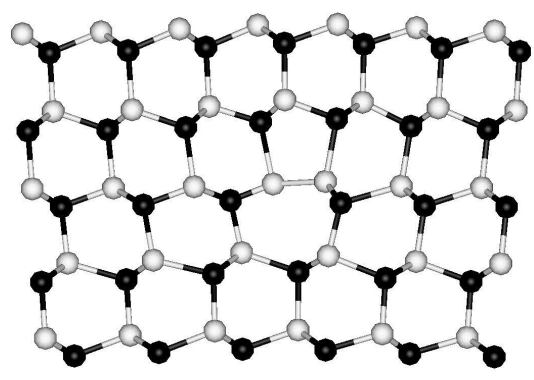

(B)

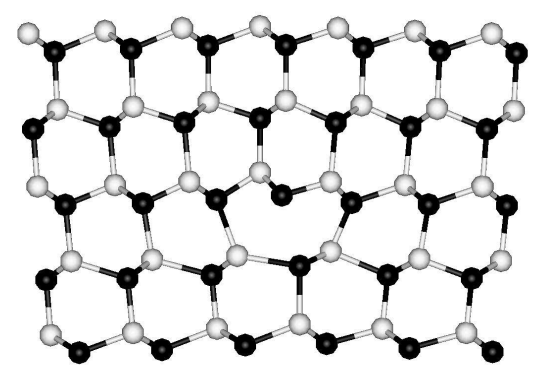

(D)

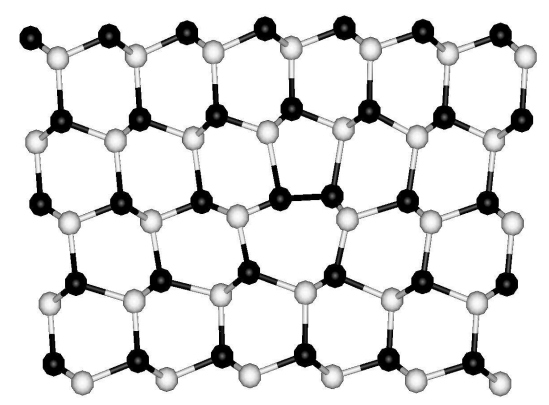

Fig. 17: The core configurations of the perfect $60^{\circ}$ dislocation in wurtzite GaN. (A) The $\alpha$ (N-terminated) shuffle configuration. (B) The $\beta$ (Ga-terminated) shuffle configuration. (C) The $\alpha$ (N-terminated) glide configuration. (D) The $\beta$ (Ga-terminated) glide configuration. Black balls represent gallium atoms and the white ones nitrogen atoms.

For the shuffle dislocations both the $\alpha$ (N-terminated) and $\beta$ (Ga-terminated) configurations were investigated. The $\alpha$ core shuffle dislocation was found to have an 8 atom rings like structure, with three fold coordinated atoms exhibiting a $\mathrm{sp}^{3}$ like hybridisation 


\subsubsection{Partial basal dislocations}

From their recent investigation, Savini et al. have reported the atomic and electronic structures of the $90^{\circ}$ Shockley partials, $(\vec{b}=a / 3[10 \overline{1} 0], l=<11 \overline{2} 0>)$, in wurtzite GaN (Fig.18) [68]. They used ab-initio calculations, implemented in the AIMPRO code, to model partial dislocations with $\alpha$ (N-terminated) and $\beta$ (Ga-terminated) cores in both supercells and cluster-supercell hybrids. By limiting their investigations to the glide configurations, they showed that the two partials can adopt both symmetric and asymmetric core reconstructions depending on the environment of the dislocation. The asymmetric reconstruction was found to 
be the most favourable and the energy difference between the two reconstructions was 83.2 $\mathrm{meV}$ for the $\beta$-core and $196.4 \mathrm{meV}$ for the $\alpha$-core. The calculated electronic structure
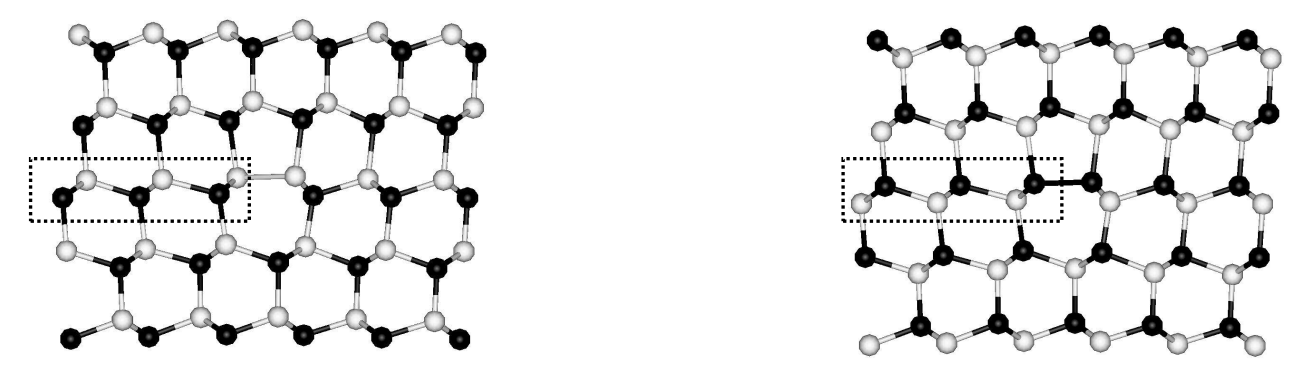

Fig.18: The core configurations of the $90^{\circ}$ Shockley partials. (A) The $\alpha$ (N-terminated) core configuration. (B) The $\beta$ (Ga-terminated) core configuration. The doted line indicates the intrinsic stacking fault. Black balls represent gallium atoms and the white ones nitrogen atoms.

exhibited mid gap states for both dislocations cores, and for the $\alpha$ core a deep acceptor level at $1.11 \mathrm{eV}$ and for the $\beta$ core a deep donor level at $0.87 \mathrm{eV}$, from the VBM, was pointed out. The first level is expected to give rise to a $2.3 \mathrm{eV}$ radiative transition which could contribute to the yellow luminescence [97], while the second one may be correlated to the $2.4 \mathrm{eV}$ absorption peak in agreement with energy loss spectroscopy measurements [98]. Based on Voronoi cell analysis of the charges density, it was shown that these partial dislocations present a charge polarization along [0001] with a negatively charged compressed region and a positively charged tensile region. Moreover, when these dislocations were brought together and in the presence of a high stress, a charge transfer was shown to occur from the $\beta$-core dislocation to the $\alpha$-core dislocation. It was concluded that such charges may be able to screen the carriers from recombination in the dislocation cores and explain why GaN devices may tolerate high dislocation densities.

In their empirical potentials investigation, Kioseoglou et al. have performed a systematic investigation of the atomic core structures and the corresponding energies of both edge 
$(\vec{b}=1 / 6[20 \overline{2} 3], l=[1 \overline{2} 10]$,$) and mixed (\vec{b}=1 / 6[2 \overline{2} 03], l=[1 \overline{2} 10])$ Frank-Shockley partials that bound the $I_{1}$ intrinsic basal stacking fault in wurtzite GaN [99]. For the edge and mixed partials, twelve stable configurations were found for each $\mathrm{Ga}$ and $\mathrm{N}$ core polarity, they consist of 5/7, 8 and 12 atom rings like structures. For the edge partials, the 5/7 atom rings structure was reported as the most energetically favourable with energies less than $0.76 \mathrm{eV} / \AA$. Whereas the 8 and 12 atom rings have energies ranging from 1.28 to $1.48 \mathrm{eV} / \AA$. For the mixed partials, both the 5/7 and 12 atom rings structures were most stable. Their core energies are larger than those of the edge dislocations and the energetically most favourable configurations have energies ranging from 0.81 to $1.05 \mathrm{eV} / \AA$. These partial dislocations should be electrically active since they present similar structures to those of the $60^{\circ}$ dislocations, already reported by Blumenau et al. in cubic GaN [95].

\section{Discussion}

The GaN layers are mostly grown on sapphire which exhibits a large mismatch, and the area in the vicinity of the interface with the substrate contains a lot of defects. As early as 1986, low temperature buffer layers (AlN, GaN) were largely used in the growth of GaN resulting in high quality epitaxial layers [100]. In these layers deposited in the 500$600^{\circ} \mathrm{C}$ temperature range, it was shown that many basal stacking faults form and during the subsequent increase of the temperature for the active layer growth they may become the origin of the threading dislocations[47]. The connection of the stacking faults and the misfit dislocations has not yet been clearly demonstrated. However, the connection between the origin of the threading dislocations and the highly strained interfacial area with substrate was established [19]. This confirms more or less the rather simple picture of mosaic growth which was published as early as 1997 [101]. Following their climb at grain boundaries or from the SF reaction area in the buffer layer, the threading dislocations obtain their equilibrium 
structure as determined by the growth kinetics. i.e.: their line becomes mainly parallel to the [0001] growth direction. The three topological dislocations of the hexagonal lattice have been observed with a vast majority of $\vec{a}$ edge ones, as reported from the start. The mosaic growth of the layers allows the $\vec{a} 60^{\circ}$ interfacial dislocations to easily become pure edge along the $c$ axis forming low angle grain boundaries. The growth along the $c$ axis limits the formation of $\vec{a}+\vec{c}<10 \%$ dislocation and even more the $\vec{c} \sim 1 \%$ as observed experimentally.

As for the atomic structure, the investigations were first misled by the fact that, even if the first layers contained $10^{10} \mathrm{~cm}^{-2}$ dislocations, the LEDs emission was the brightest known in semiconductor optoelectronics.

The multiplicity of the atomic configurations was shown as early as 1998 with the coexistence of the 5/7 and 8 atom ring cores in good quality GaN layers types[13] and the occurrence of the additional 4 atoms core inside grain boundaries[18]. Of course, as it was demonstrated later on, the three configurations are just a consequence of the wurtzite structure, and the occurrence of each depends on the position of the dislocation line inside the unit cell [21]. Then, by using our empirical potential [75] along with ab initio calculations, Lymperakis at al. have carried out a multiscale analysis of this edge threading dislocation [23]. They convincingly demonstrated that, the 4 atom ring core which has been first reported in grain boundaries may be the most stable in strained layers [18].

At the initial stages of the growth, many stacking faults are involved and partial dislocations can be present. However, it is of interest to investigate their atomic structure. It is may be noticed that as stressed by Narayan et al. [47], they will quickly develop in threading dislocations and behave like the dislocations $\vec{a}, \vec{a}+\vec{c}$ and $\vec{c}$ of the hexagonal lattice.

The electronic structure of the threading dislocations has been extensively debated and now the situation is clearer. For the prismatic edge dislocation with full or open core, empty states are present in the half top of the band gap. Some discrepancies still remain about their 


\section{Conclusion}

In this paper, we presented and discussed our results dealing with the dislocations in GaN layers. The dislocations are strongly involved in degrading the performance of the devices. The atomic core structures of the perfect edge threading dislocations are based on only three types of atom rings, however, depending on the layer growth conditions, they can be stoichiometric or not. The perfect dislocations, threading and basal dislocations, have now been largely investigated unlike the partial dislocations. Their electrical activity is in good 
agreement with their atomic structure. A number of theoretical reports show that their electronic structures exhibit deep and shallow levels.

Acknowledgements: The authors acknowledge the support of the EU under contracts "IPAM" HPRN-CT-1999-00040, and "PARSEM" MRTN-CT-2004-005583. The computations were performed at "CRIHAN" (http://www.crihan.fr).

\section{References}

[1] S. Nakamura, T. Mukai and M. Senoh, Appl. Phys. Lett. 641687 (1994).

[2] J. Elsner, R. Jones, P. K. Sitch, V. D. Porezag, M. Elstner, Th. Frauenheim, M. I. Heggie, S. Öberg and P. R. Briddon, Phys. Rev. Lett., 793672 (1997).

[3] F.R. Chien, X.J. Ning, S. Stemmer, P. Pirouz, M.D. Bremser and R.F. Davis, Appl. Phys. Lett., 682678 (1996).

[4] X.H. Wu, L.M. Brown, D. Kapolnek, S. Keller, B. Keller, S.P. DenBaars, and J.S. Speck, J.Appl. Phys., 803228 (1996).

[5] A.F. Wright and J.S. Nelson, Phys.Rev.B, 517866 (1995).

[6] L. Sigiura, J. Appl. Phys., 811633 (1997).

[7] Y. Xin, S. J. Pennycoock, N.D. Browning, P.D. Nelist, S. Sivananthan, B. Beaumont, J. P. Faurie and P. Gibart, Mat. Res. Soc. Symp. Proc., 482781 (1998).

[8] P. Vermaut, P. Ruterana, G. Nouet and H. Morkoç, Phil. Mag. A, 75239 (1997).

[9] B. Daudin, J. L Rouviere. and M. Arlery, Appl.Phys.Lett., 692480 (1996).

[10] V. Potin, P. Vermaut, P. Ruterana and G. Nouet, J. Electron. Mater., 27266 (1998).

[11] N.G. Weimann, L.F. Eastman, D. Doppalapudi, H.M. Ng and T.D. Moustakas, J. Appl. Phys., 833656 (1998).

[12] S. Nakamura and G. Fasol, The Blue Laser Diodes (Springer, Heidelberg, 1997).

[13] P. Ruterana, V. Potin and G. Nouet, Mat. Res. Soc. Symp. Proc., 482459 (1998).

[14] A.F. Wright, Mat. Res. Soc. Symp. Proc., 482795 (1998). 
[15] J. E Northrup., Appl. Phys. Lett., 782288 (2001).

[16] J. E. Northrup, Phys. Rev. B, 66045204 (2002).

[17] I. Arslan and N.D. Browning, Phys.Rev. Lett., 91165501 (2003).

[18] V. Potin, P. Ruterana, G. Nouet, R.C. Pond and H. Morkoç, Phys. RevB., 615587 (2000).

[19] G.P. Dimitrakopulos, Ph. Komninou, J. Kioesoglou, Th. Kehagias, E. Sarigiannidou, G A.Georgakilas, G. Nouet and Th. Karakostas, Phys. Rev. B, 64245325 (2001).

[20] J. Chen, P. Ruterana and G.Nouet, Mater.Sci.Eng. B, 82117 (2001).

[21] A. Béré, J. Chen, P. Ruterana, A. Serra and G. Nouet, Compt. Mat. Sci., 24144 (2002).

[22] A.T. Blumenau, C.J. Fall, J. Elsner, R. Jones, M.I. Heggie and Th. Frauenheim, phys.stat.sol.(c), 01684 (2003).

[23] L. Lymperakis, J. Neugebauer, M. Albrecht, T. Remmele and H.P.Strunk, Phys. Rev. Lett., 93 196401(2004).

[24] D.B. Holt, J. Mater.Sci., 231131 (1988).

[25] T. Mattila and A. Zunger, J. Appl. Phys., 85160 (1999).

[26] J.P. Hirth and J. Lothe, Theory of dislocations (Wiley, New York, 1982).

[27] F.A. Ponce, D. Cherns, W.T. Young and J.W. Steeds, Appl. Phys. Lett., 69770 (1996).

[28] P. Gibart, B. Beaumont and P. Vennéguès, Nitride Semiconductors Handbook on Materials and Devices, edited by P. Ruterana, M.Albrecht, J. Neugebauer (Wiley-VCH, 2003), pp. 45.

[29] V. Potin, PhD Thesis, University of Caen (1999).

[30] P.A. Stadelmann, Ultramicroscopy, 21131 (1987).

[31] Y. Xin, S.J. Pennycoock, N.D. Browning, P.D. Nelist, S. Sivananthan, F. Omnès, B. Beaumont, J. P. Faurie and P. Gibart, Appl. Phys. Lett., 722680 (1998).

[32] V. Potin, P. Ruterana, A. Hairie and G. Nouet, Mat. Res. Soc. Symp. Proc., 482435 (1998).

[33] R.C. Pond, Dislocations in Solid, edited by F.R.N. Nabarro (North Holland, Amsterdam 1989) 8 pp.1.

[34] W. Bollmann, Crystal Defects and Crystalline Interface, (Springer, Berlin, 1970).

[35] J. Chen, P. Ruterana and G. Nouet, Phys. Rev. B, 67205210 (2003). 
[36] P. Ruterana, A.M. Sanchez and G. Nouet, Nitride Semiconductors Handbook on Materials and Devices, edited by P. Ruterana, M. Albrecht, J. Neugebauer (Wiley-VCH, 2003), pp. 379.

[37] H.M. Hobgood, D.L. Barrett, J.P. McHugh, R.C. Clarke, S. Sriram, A.A. Burk, J. Greggi, C.D. Brandt, R.H. Hopkins and W.J. Choyke, J. Cryst. Growth, 137181 (1994).

[38] Z. Liliental-Weber, Y. Chen, S. Ruvimov and J. Washburn, Phys. Rev. Lett., 792835 (1997).

[39] W. Qian, G. S. Rohrer, M. Skowrnonski, K. Doverspike, L. B. Rowland and D.K. Gaskill, Appl. Phys. Lett., 672284 (1995).

[40] P. Vennegues, B. Baumont, M. Vaille and P. Gibart, Appl. Phys. Lett., 702434 (1997).

[41] J.W.P. Hsu, M.J. Manfra, R.J. Molnar, B. Heying and J. S. Speck, Appl. Phys. Lett., 81 79 (2002).

[42] Z. Liliental-Weber, D. Zakharov, J. Jasisnski, J.Washburn, M.A. O'Keefe and H. Morkoç., Mat. Res. Soc. Sym. Proc., 743243 (2003).

[43] P. Kung, C.J. Sun, A. Saxler, H. Ohsato and M. Razeghi, J. Appl. Phys., 754515 (1994).

[44] S. Kaiser, H. Preis, W. Gebhart, O. Ambacher, H. Angerer, M. Stutzmann, A. Rosenauer and D. Gerthsen, Jpn. J. Appl. Phys., 3784 (1998).

[45] Th. Kehagias, Ph. Komninou, G. Nouet, P. Ruterana and Th. Karakostas, Phys. Rev.B, 64195329 (2001).

[46] L.A. Bendersky, D.V. Tsvetkov and Y.V. Melnik, J. Appl. Phys., 941676 (2003).

[47] V. Narayan, K. Lorenz., W. Kim and S. Mahajan, Appl. Phys. Lett. 781544 (2001).

[48] M. Albrecht, H.P.Strunk, J.L. Weyher, I. Grzegory, S. Porowsky and T.Wosinski, J. Appl.Phys., 922000 (2002).

[49] C. Stampfl and C.G. Van de Walle, Phys. Rev. B, 57 R15052 (1998).

[50] J.A. Chislom and P.D. Bristowe, J. Phys.: Condens. Matter., 115067 (1999).

[51] P. Ruterana and G. Nouet, phys.stat.sol.(b), 227177 (2001).

[52] I. Arslan, A. Bleloch, E.A. Stach and N.D. Browning, Phys.Rev. Lett., 94, 025504 (2005).

[53] J.W.P. Hsu, M.J. Manfra, S.N.G. Chu, C.H. Chen, L.N. Pfeiffer and R.J. Molnar, Appl.Phys.Lett., 783980 (2001). 
[54] T. Hino, S. Tomiya, T. Miyajima, K. Yanashima, S. Hashimoto, and M. Ikeda, Appl. Phys. Lett., 763421 (2000).

[55] E. G. Brazel, M. A.Chin, and V. Narayanamurti, Appl. Phys. Lett., 74, 2367 (1999).

[56] T. Sugahara, H.Sato, M. Hao, Y. Naoi, S.Kurai, S.Tottori, K. Yamashita, K. Nishino, L. Romano and S. Sakai, Jpn. J. Appl. Phys., 37 L398 (1998).

[57] T. Remmele, M. Albrecht, H.P. Strunk, A.T. Blumenau, M.I. Heggie, J. Elsner, F T. Frauenheim, H.P.D. Schenk and P. Gibart, Inst. Phys. Conf. , 169, 323 (2001).

[58] W.J. Tunstall, P.B. Hirsch. and J. Steeds, Phil. Mag. 9, 99 (1964).

[59] P. Hohenberg and W. Kohn, Phys. Rev. B, 136864 (1964).

[60] W. Kohn and L. Sham, Phys. Rev. A, 1401133 (1965).

[61] W.E. Pickett, Computer Physics Reports, 9115 (1989).

[62] G. Kresse and J. Furthmüller, Phys. Rev. B, 5411169 (1996).

[63] M. Bockstedte, A.Kley, J. Neugebauer and M. Scheffler, Comp.Phys.Commun., 107187 (1997).

[64] P.R. Briddon and R. Jones, phys.stat.sol.(b), 217131 (2000).

[65] J.M. Soler, E. Artacho, J. Gale, A. Garcia, J. Junquera, P. Ordejon and D. SanchezPortal, J. Phys.: Condens. Matter, 142745 (2002).

[66] A.F. Wright and U. Grossner, Appl. Phys. Lett., 732751 (1998).

[67] C.J. Fall, R. Jones, P. Briddon, A.T. Blumenau, Th. Frauenheim, M.I. Heggie, Phys. Rev. B, 65245304 (2002).

[68] G. Savini, M.I. Heggie, C.P. Ewels, N. Martsinovich, R. Jones and A.T. Blumenau, , Material Science Forum, 483-485 1057 (2005).

[69] M. Finnis, Forces in Condensed Matter (Oxford University Press, Oxford, 2003).

[70] P.N. Keating, Phys. Rev., 145637 (1966).

[71] G.A. Baraff, E.D. Kane and M.Schluter, Phys.Rev. B, 215665 (1980).

[72] F.H. Stillinger and T.A. Weber, Phys. Rev. B, 315262 (1985).

[73] J. Tersoff, Phys.Rev.B, 376991 (1988); Phys.Rev.B, 389902 (1988); Phys.Rev.Lett., 61 2879 (1988); Phys.Rev.B, 395566 (1989).

[74] M. Ichimura, phys. stat. sol. (a), 153431 (1996). 
[75] N. Aïchoune, V. Potin, P. Ruterana, A. Hairie, G. Nouet and E. Paumier, Comput. Mater. Sci., 17380 (2000).

[76] A. Béré and A. Serra, Phys. Rev.B, 66085330 (2002).

[77] J. Kioseoglou, H.M. Polatoglou, L. Lymperakis, G. Nouet and Ph. Komninou, Comp. Mater. Sci., 2743 (2003).

[78] I. Belabbas, J. Chen, M.A. Belkhir, P. Ruterana, and G. Nouet, paper presented at Symposium F of the E-MRS Fall Meeting, Warsaw, Poland, 2-5 September (2005).

[79] D.J. Chadi, Phys. Rev. Lett., 4379 (1979).

[80] W.M.C. Foulkes and R. Haydock, Phys. Rev. B, 3912520 (1989).

[81] J.C. Slater and G.F. Koster, Phys. Rev., 941498 (1954).

[82] A.P. Horsfield and A.M. Bratkovsky, J.Phys.:Condens. Matter., 12 R1 (2000).

[83] M. Elstner, D. Porezag, G. Jungnickel, J.Elsner, M. Haugk, T. Frauenheim, S.Suhai, and G. Geifert, Phys. Rev. B 587260 (1998).

[84] T. Frauenheim, G. Seifert, M. Elstner, Z. Hajnal, G. Jungnickel, D. Porezag, S. Suhai, and R. Scholz, phys. stat. sol.( b), 21741 (2000).

[85] T. Frauenheim, G. Seifert, M. Elstner, T. Niehaus, C. Kohler, M. Amkreutz, M. Sternberg, Z. Hajnal, A. Di Carlo and S. Suhai, J. Phys.: Condens. Matter, 143015 (2002).

[86] D.Porezag, Th. Frauenheim, and Th. Köhler, Phys. Rev. B 5112947 (1995).

[87] M. Haugk, J. Elsner, T. Frauenheim, T.E.M. Staab, C.D. Latham, R. Jones, H. S.Leipner, T. Heine, G. Seifert and M. Sternberg, phys. stat. sol.(b), 217473 (2000).

[88] S.M. Lee, M.A.Belkhir, X.Y. Zhu, Y.H. Lee, Y.G. Hwang and Th. Frauenheim, Phys. Rev. B, 6116033 (2000).

[89] I.Belabbas, M.A. Belkhir, Y.H. Lee, A. Béré, P. Ruterana, J. Chen, and G. Nouet, phys. stat. sol. (c), 22492 (2005).

[90] A. Béré, P. Ruterana, G. Nouet, A.T. Blumenau, S. Sanna, T. Frauenheim, J.Chen and J. Koulidiati, Phys. Rev.B, 71125211 (2005).

[91] A. Béré, J. Chen, G. Nouet, P. Ruterana and J. Koulidiati, phys.stat.sol.(c), 22496 (2005).

[92] K. Leung, A.F. Wright and E.B. Stechel, Appl. Phys. Lett., 742495 (1999). 
[93] J. Chen, P. Ruterana and G. Nouet, presented at Symposium F of the E-MRS Fall Meeting, Warsaw, Poland, 2-5 September (2005).

[94] P. Vennegues, B. Beaumont, V. Bousquet, M. Vaille and P. Gibart, J. Appl. Phys., 87 $4175(2000)$.

[95] B.A. Haskell, F. Wu, S. Matsuda, M.D. Craven, P.T. Fini, S. Den Baars, J.S. Speck and S. Nakamura, Appl. Phys. Lett., 831554 (2003).

[96] D.J. As, A. Richter, J.Busch, B. Schottker, M. Lubbers, J. Mimkes, D. Schikora, K. Lischka, W. Kriegseis, W. Burkhardt and B.K. Meyer, MRS Internet J. Nitride Semicond. Res., 5S1 W3.81 (2000).

[97] X.G. Qiu, Y.Segawa, Q.K. Xue, Q.Z. Xue and T. Sakurai, Appl. Phys. Lett., 771316 (2000).

[98] A. Gutierrez-Sosa, U. Bangert, A.J.Harvey, C.J. Fall, R. Jones, P.R. Briddon and M.I. Heggie, Phys. Rev. B, 6635302 (2002).

[99] J. Kioseoglou, G.P. Dimitrakopulos, Ph. Komninou and Th.Karakostas, Phys. Rev. B, 70 035309 (2004).

[100] H. Amano, N. Sawaki, I. Akasaki and Y. Toyoda, Appl. Phys. Lett., 48353 (1986).

[101] F.A. Ponce and D.P. Bour, Nature, 386, 351 (1997). 